\title{
ALGORITHMS FOR MATRIX EXTENSION AND ORTHOGONAL WAVELET FILTER BANKS OVER ALGEBRAIC NUMBER FIELDS
}

\author{
BIN HAN AND XIAOSHENG ZHUANG
}

\begin{abstract}
As a finite dimensional linear space over the rational number field $\mathbb{Q}$, an algebraic number field is of particular importance and interest in mathematics and engineering. Algorithms using algebraic number fields can be efficiently implemented involving only integer arithmetics. We observe that all known finitely supported orthogonal wavelet low-pass filters in the literature have coefficients coming from an algebraic number field. Therefore, it is of theoretical and practical interest for us to consider orthogonal wavelet filter banks over algebraic number fields. In this paper, we formulate the matrix extension problem over any general subfield of $\mathbb{C}$ (including an algebraic number field as a special case), and we provide step-by-step algorithms to implement our main results. As an application, we obtain a satisfactory algorithm for constructing orthogonal wavelet filter banks over algebraic number fields. Several examples are provided to illustrate the algorithms proposed in this paper.
\end{abstract}

\section{INTRODUCTION AND MOTIVATION}

In a digital world, data and signals are often recorded using integers and dyadic rational numbers. For example, a 8-bit grey-scale image has its pixels taking integer values between 0 and 255. The rational number field $\mathbb{Q}$ has many advantages in scientific computing. Using integer arithmetics and having simple hardware implementation, algorithms using the rational number field are much more efficient than using floating point arithmetics, and avoid roundup error in computation. Consequently, it is one of the fascinating topics in applied mathematics and engineering (see [3, 16, 19]) to construct orthogonal wavelet filter banks with rational coefficients. Indeed, a few examples of orthogonal wavelet low-pass filters with rational coefficients have been reported in [1, 2, 3, 5. Typically, the associated wavelets in such examples have pretty low regularity and vanishing moments. This significantly limits the use of such orthogonal wavelet low-pass filters in applications. The interest of seeking orthogonal wavelet filter banks with rational coefficients has been renewed recently in the paper Mo and $\mathrm{Li}$ [16, where tight framelet filter banks with rational coefficients and with two high-pass filters have been considered. Though a necessary and sufficient condition is obtained in [16] for a dyadic tight framelet

Received by the editor April 9, 2011 and, in revised form, September 7, 2011.

2010 Mathematics Subject Classification. Primary 42C40, 41A05; Secondary, 42C15, 65T60.

Key words and phrases. Matrix extension, matrix factorization, algebraic number fields, symmetry, multiwavelets, orthogonal wavelet filter banks, algebraic wavelet filters.

This research was supported in part by the Natural Sciences and Engineering Research Council of Canada (NSERC Canada) under Grant RGP 228051. 
filter bank with two high-pass filters having rational coefficients, almost none of the known examples in the literature can satisfy the necessary and sufficient condition in [16]. Moreover, the associated wavelets in the illustrative examples presented in [16] have only one vanishing moment. Note that a tight framelet filter bank is a generalization of an orthogonal wavelet filter bank by having more than necessary number of high-pass filters. Despite the interesting work in 16, constructing orthogonal wavelet filter banks or tight framelet filter banks with rational coefficients and with high vanishing moments remains as a challenging task.

We are motivated by [16] to examine again all the orthogonal wavelet filter banks available in the literature. We observe two interesting phenomena. First, though there are very few known examples of orthogonal wavelet low-pass filters with rational coefficients, we notice that to our best knowledge all known finitely supported orthogonal wavelet low-pass filters have their coefficients coming from an algebraic number field, often in the form $\mathbb{Q}\left(\sqrt{t_{1}}, \ldots, \sqrt{t_{n}}\right)$ for some positive integers $t_{1}, \ldots, t_{n}$. For example, see [8] and [1, 2, 15, 13] for many examples of orthogonal wavelet low-pass filters with symmetry. We shall provide a natural explanation in Section 3 for this observation. Recall that an algebraic number field $\mathbb{A}$ is a finite field extension of the rational number field $\mathbb{Q}$. More precisely, $\mathbb{A}=\mathbb{Q}\left(t_{1}, \ldots, t_{n}\right)$, where each of $t_{1}, \ldots, t_{n}$ is a root of some polynomial with integer coefficients. It is well known that an algebraic number field $\mathbb{A}$, when viewed as a linear space over $\mathbb{Q}$, is a finite dimensional vector space over $\mathbb{Q}$. Consequently, the arithmetics over $\mathbb{A}$ can be implemented by combining the integer arithmetics and matrix/vector operations from linear algebra (for example, see [14 for more detail). In other words, algorithms over an algebraic number field $\mathbb{A}$ have the same order of complexity as those over the rational number field $\mathbb{Q}$. Consequently, it is of theoretical and practical interest for us to consider orthogonal wavelet filter banks over an algebraic number field.

Second, we observe that even though the orthogonal wavelet low-pass filters constructed in [1, Examples 1 and 2] and 2, (5.3)] have rational coefficients, the constructed high-pass filters, which are obtained by a brute force calculation using Gröbner bases, do not have rational coefficients; instead, such derived high-pass filters have rational coefficients only up to a multiplicative constant $\sqrt{t}$ for some positive integer $t$. In other words, according to the evidence from symbolic computation, even if an orthogonal wavelet low-pass filter has rational coefficients, the derived high-pass filters cannot always be expected to have rational coefficients. This motivates us to examine the classical matrix extension problem in [6, 9, 10, 15, 17, 19] and to seek the right formulation for the matrix extension problem and for the construction of orthogonal wavelet filter banks over an algebraic number field.

In the following, we shall state our formulation of the matrix extension problem over a general field including an algebraic number field as special cases. To do so, let us recall some notation and definitions. Let $\mathbb{F}$ denote a general subfield of $\mathbb{C}$ such that

$$
\bar{x} \in \mathbb{F} \quad \text { if } \quad x \in \mathbb{F},
$$

where $\bar{x}$ denotes the complex conjugate of a complex number $x \in \mathbb{C}$. That is, the subfield $\mathbb{F}$ is closed under complex conjugation. For example, $\mathbb{F}$ can be the rational number field $\mathbb{Q}$ or an algebraic number field $\mathbb{A}$ satisfying $\bar{x} \in \mathbb{A}$ for all $x \in \mathbb{A}$. By $\mathbb{F}\left[z, z^{-1}\right]$ we denote the set of all Laurent polynomials with coefficients in $\mathbb{F}$. We 
shall use the following notation throughout the paper:

$$
\mathrm{P}^{\star}(z):=\mathrm{P}(z)^{\star}:=\sum_{k \in \mathbb{Z}}{\overline{P_{k}}}^{\top} z^{-k} \quad \text { for } \quad \mathrm{P}(z)=\sum_{k \in \mathbb{Z}} P_{k} z^{k},
$$

where $P_{k} \in \mathbb{C}^{r \times s}$ are $r \times s$ matrices of complex numbers. We also define $P_{k}^{\star}:={\overline{P_{k}}}^{\top}$ for a matrix $P_{k}$ of complex numbers. With the above $\star$ notation, we often work on $\mathrm{P}(z)$ with $z \in \mathbb{T}:=\{\zeta \in \mathbb{C}|| \zeta \mid=1\}$.

Now we are ready to formulate the matrix extension problem over a general field $\mathbb{F}$. Let $\mathrm{P}$ be an $r \times s$ matrix, with $1 \leqslant r \leqslant s$, of Laurent polynomials satisfying

(i) $\mathrm{P}$ is paraunitary: $\mathrm{P}(z) \mathrm{P}^{\star}(z)=I_{r}$ for all $z \in \mathbb{C} \backslash\{0\}$;

(ii) $\mathrm{P}$ takes the form $\mathrm{P}(z)=\mathrm{Q}_{0}(z) D_{0}$, where $\mathrm{Q}_{0}$ is an $r \times s$ matrix of Laurent polynomials in $\mathbb{F}\left[z, z^{-1}\right]$ and $D_{0}$ is a diagonal matrix with all the entries of $D_{0} D_{0}^{\star}$ in $\mathbb{F}$.

The matrix extension problem over a general field $\mathbb{F}$ is to find an $s \times s$ extension matrix $\mathrm{P}_{e}$ of Laurent polynomials such that

(1) $\left[I_{r}, \mathbf{0}\right] \mathrm{P}_{e}=\mathrm{P}$; that is, the submatrix of the first $r$ rows of $\mathrm{P}_{e}$ is $\mathrm{P}$;

(2) $\mathrm{P}_{e}$ is paraunitary: $\mathrm{P}_{e}(z) \mathrm{P}_{e}^{\star}(z)=I_{s}$ for all $z \in \mathbb{C} \backslash\{0\}$;

(3) $\mathrm{P}_{e}$ takes the form $\mathrm{P}_{e}(z)=D_{e} \mathrm{Q}_{e}(z) D_{0}$, where $\mathrm{Q}_{e}$ is an $s \times s$ matrix of Laurent polynomials in $\mathbb{F}\left[z, z^{-1}\right]$ and $D_{e}$ is a diagonal matrix with all the entries of $D_{e} D_{e}^{\star}$ in $\mathbb{F}$.

Moreover, if the given matrix $\mathrm{P}$ has certain symmetry structure, then it is desirable that the extension matrix $\mathrm{P}_{e}$ also possesses certain symmetry structure. It is also important that the support of the coefficients of $\mathrm{P}_{e}$ is controllable in some way by that of the given matrix $P$.

We shall explain in Section 3 why the above formulation of the matrix extension problem over a general field $\mathbb{F}$ leads to a satisfactory solution in the setting of wavelet analysis and filter banks.

The classical formulation of the matrix extension problem corresponds to the special case $D_{0}=D_{e}=I_{s}$, but only for subfields $\mathbb{F}$ of $\mathbb{C}$ satisfying the following property:

$$
\bar{x} \in \mathbb{F} \quad \text { and } \quad \sqrt{y} \in \mathbb{F} \quad \forall x, y \in \mathbb{F} \quad \text { with } \quad y>0 .
$$

For example, in the context of wavelet analysis and filter banks, the matrix extension problem without symmetry for $\mathbb{F}=\mathbb{R}$ or $\mathbb{C}$ was studied in Lawton, Lee, and Shen [15] and Vaidyanathan [19. The matrix extension problem with symmetry has been considered in Petukhov [17] for $r=1$ and $\mathbb{F}=\mathbb{R}$, and in Han [6] for $r=1$ and a general subfield $\mathbb{F}$ of $\mathbb{C}$ satisfying (1.2). More recently, the matrix extension problem with symmetry has been studied in [10] by the authors for any $r$ satisfying $1 \leqslant r \leqslant s$ and any subfield $\mathbb{F}$ of $\mathbb{C}$ satisfying the condition in (1.2). Clearly, the condition in (1.2) is satisfied if $\mathbb{F}=\mathbb{R}$ or $\mathbb{C}$ and therefore, 10 generalizes the results in [6, 17] to the most general setting.

However, even if all the coefficients of $\mathrm{P}$ are integers, the smallest such field $\mathbb{F}$, containing $\mathbb{Z}$ and satisfying the condition in (1.2), must contain $\sqrt{n}$ for all positive integers $n$. Therefore, any subfield $\mathbb{F}$ satisfying (1.2) can never be an algebraic number field and it must be an infinite dimensional linear space over $\mathbb{Q}$. Hence, in the setting of wavelet analysis and filter banks, despite the beautiful results and efficient algorithms on the matrix extension problem in [6, 10, 15, 17, 19, even if an orthogonal wavelet low-pass filter has all its coefficients from an algebraic number 
field (which is indeed the case for all known finitely supported orthogonal wavelet low-pass filters in the literature, for example, see 8 for systematic construction and numerous examples of orthogonal wavelet low-pass filters with symmetry), the derived orthogonal wavelet high-pass filters cannot be guaranteed to have coefficients in an algebraic number field as well.

For a matrix $\mathrm{P}(z)=\sum_{k=m}^{n} P_{k} z^{k}$ of Laurent polynomials such that $P_{m} \neq \mathbf{0}$ and $P_{n} \neq \mathbf{0}$ with $m, n \in \mathbb{Z}$, we define the filter support and length of $\mathrm{P}$ to be $\operatorname{fsupp}(\mathrm{P}):=[m, n]$ and $\operatorname{len}(\mathrm{P}):=n-m$, respectively. Throughout the paper, $\mathbf{0}$ always denotes a zero matrix whose size can be determined from the context.

Let $\mathrm{P}$ be an $r \times s$ matrix, with $1 \leqslant r \leqslant s$, of Laurent polynomials satisfying $\mathrm{P}(z) \mathrm{P}^{\star}(z)=I_{r}$ for all $z \in \mathbb{C} \backslash\{0\}$ and $\mathrm{P}$ takes the form $\mathrm{P}(z)=\mathrm{Q}_{0}(z) D_{0}$, where $\mathrm{Q}_{0}$ is an $r \times s$ matrix of Laurent polynomials in $\mathbb{F}\left[z, z^{-1}\right]$ and $D_{0}$ is a diagonal matrix with all the entries of $D_{0} D_{0}^{\star}$ in $\mathbb{F}$. To study and formulate the matrix extension problem over a general subfield $\mathbb{F}$, in this paper we shall investigate the structure of $\mathrm{P}$ by factorizing the matrix $\mathrm{P}$ into a product of elementary matrices, that is, we study a more general problem: the matrix factorization problem over a general subfield $\mathbb{F}$ of $\mathbb{C}$. More precisely, we shall construct a sequence of elementary matrices $A_{0}, \ldots, A_{J}$ such that

(1) $\mathrm{PA}_{0} \cdots \mathrm{A}_{J}=\left[I_{r}, 0\right]$;

(2) len $\left(\mathrm{PA}_{0} \cdots \mathrm{A}_{j}\right)<\operatorname{len}\left(\mathrm{PA}_{0} \cdots \mathrm{A}_{j-1}\right)$ for all $j=0, \ldots, J$;

(3) $\mathrm{A}_{j}^{\star}(z) \mathrm{A}_{j}(z)=I_{s}$ for all $j=0, \ldots, J$ and $\operatorname{len}\left(\mathrm{A}_{j}\right) \leqslant 1$;

(4) Each $\mathrm{A}_{j}$ takes the form $D_{j-1}^{\star} \vee_{j}(z) D_{j}$ with $\vee_{j}(z)=V_{j} \mathrm{D}_{j}(z)$, where all $D_{j}$ are diagonal matrices such that all entries of $D_{j}^{\star} D_{j}$ in $\mathbb{F}, V_{j}$ is a constant invertible matrix in $\mathbb{F}$, and $\mathrm{D}_{j}$ is a diagonal matrix whose diagonal entries are monomial Laurent polynomials.

Consequently, we have the following representation of $\mathrm{P}: \mathrm{P}=\left[I_{r}, \mathbf{0}\right] \mathrm{A}_{J}^{\star} \cdots \mathrm{A}_{0}^{\star}$, which factorizes the paraunitary matrix $P$ into a product of elementary paraunitary matrices $\mathrm{A}_{j}^{\star}$. When $\mathrm{P}$ has symmetry, the matrix extension problem and the matrix factorization problem over a general subfield $\mathbb{F}$ are even much more complicated; see Section 4 for details. The matrix extension problem and the matrix factorization problem are of importance in engineering and system sciences. For example, they play a central role in engineering for the design and study of paraunitary perfect reconstruction filter banks (see [19, Sections 13 and 14]); they are also indispensable tools in the general theory of linear systems in system science; see [20] and references therein.

Our major contribution of this paper is a proper formulation of the matrix extension problem and the matrix factorization problem with or without symmetry over a general field, and provides a satisfactory solution to them. Though we shall use some interesting ideas from [6, 10, 15, 17, 19], our results in this paper are not simple generalizations of the results in [6, 10, 15, 17, 19] and new ideas are needed in order to satisfactorily solve the matrix extension problem over a general field without the condition in (1.2). More precisely, to avoid the square root requirement of the subfield $\mathbb{F}$ in (1.2), we have to formulate the matrix extension and factorization problems in an appropriate way by restricting ourselves to considering only elementary matrices with additional structures. Our main new idea is a key observation made in Lemma 2.2 which allows us to avoid directly using the square roots of positive elements in $\mathbb{F}$ in our elementary matrices. Instead, we require that elementary matrices should have the additional structure $D_{0}^{\star} V D_{1}$ such that $V$ is 
an invertible matrix in $\mathbb{F}$ and $D_{0}, D_{1}$ are diagonal matrices whose diagonal entries are square roots of positive elements in $\mathbb{F}$. In this way, the troubling square roots of positive elements in $\mathbb{F}$ appearing in our elementary matrices are restricted in the diagonal matrices $D$ 's in a nested fashion (see Theorem 2.1 for details). On the other hand, all the papers [6, 10, 15, 19] employ the Householder matrices to transform a constant vector into a normalized one with only one nonzero entry. However, using Householder matrices, one has to unavoidably use the square roots of positive elements in $\mathbb{F}$ in elementary matrices in a nonseparable way, which in turn forces one to use a subfield $\mathbb{F}$ with the square root requirement in (1.2). Once we have the key observation made in Lemma 2.2. to establish the results and algorithms in this paper, we shall follow the main ideas made in [6, 10, by appropriately modifying the key procedures in [6, 10, to the new setting and formulation.

The structure of the paper is as follows. In Section 2, we shall state our result on the matrix extension problem without symmetry constraint over a general field and its associated step-by-step algorithm for deriving the extension matrix $\mathrm{P}_{e}$ from P. In Section 3, we will discuss the application of the matrix extension problem to the orthogonal wavelet filter banks without symmetry. We shall explain in Section 3 that our formulation of the matrix extension problem over a general field $\mathbb{F}$ leads to a satisfactory solution in the setting of wavelet analysis and filter banks. In Section 4, we shall present our result on the matrix extension problem with symmetry constraint over a general field and its associated step-by-step algorithm for deriving the extension matrix $\mathrm{P}_{e}$ with symmetry structure from $\mathrm{P}$. In Section 5 , we shall discuss the application of our results on the matrix extension problem over a general field to wavelet analysis and filter banks. We shall see that our result leads to a satisfactory solution to the design of symmetric filter banks over an algebraic number field. We shall also provide several examples in Section 6 to demonstrate our algorithms for designing orthogonal wavelet filter banks over an algebraic number field. Proofs of some key lemmas are postponed to Section 7.

\section{The MATRIX EXTEnsion PROBLEM Without SYMMETRY CONSTRAINT}

In this section, we shall investigate the matrix extension problem without symmetry constraint over a general subfield $\mathbb{F}$ of the complex number field $\mathbb{C}$. We also present a step-by-step algorithm for finding the extension matrix $\mathrm{P}_{e}$. To do so, let us introduce some notation first.

Without further mention, $\mathbb{F}$ always denotes a general subfield of $\mathbb{C}$ satisfying (1.1). We have the following result on the matrix extension problem without symmetry constraint over a general subfield $\mathbb{F}$ :

Theorem 2.1. Let $\mathrm{P}$ be an $r \times s$ matrix, with $1 \leqslant r \leqslant s$, of Laurent polynomials such that $\mathrm{P}$ takes the following form:

$$
\mathrm{P}(z)=\mathrm{Q}_{0}(z) D_{0}, \quad z \in \mathbb{C} \backslash\{0\},
$$

where $\mathrm{Q}_{0}$ is an $r \times s$ matrix of Laurent polynomials in $\mathbb{F}\left[z, z^{-1}\right]$ and $D_{0}$ is an $s \times s$ diagonal matrix with all the entries of $D_{0} D_{0}^{\star}$ in $\mathbb{F}$. Then $\mathrm{P}(z) \mathrm{P}^{\star}(z)=I_{r}$ for all $z \in \mathbb{C} \backslash\{0\}$, if and only if, there exists an $s \times s$ matrix $\mathrm{P}_{e}$ of Laurent polynomials such that

(i) $\left[I_{r}, \mathbf{0}\right] \mathrm{P}_{e}=\mathrm{P}$; that is, the submatrix of the first $r$ rows of $\mathrm{P}_{e}$ is $\mathrm{P}$;

(ii) $\mathrm{P}_{e}$ is paraunitary: $\mathrm{P}_{e}(z) \mathrm{P}_{e}^{\star}(z)=I_{s}$ for all $z \in \mathbb{C} \backslash\{0\}$; 
(iii) $\mathrm{P}_{e}$ can be represented as a product of $s \times s$ elementary matrices:

$$
\mathrm{P}_{e}(z)=D_{e} \mathrm{P}_{J}(z) \mathrm{P}_{J-1}(z) \cdots \mathrm{P}_{1}(z) \mathrm{P}_{0}(z) D_{0}, \quad z \in \mathbb{C} \backslash\{0\},
$$

where $\mathrm{P}_{j}, j=0, \ldots, J$ are $s \times s$ matrices of Laurent polynomials in $\mathbb{F}\left[z, z^{-1}\right]$ such that $\operatorname{len}\left(\mathrm{P}_{j}\right) \leqslant 1$, and $D_{e}$ is an $s \times s$ diagonal matrix with all the entries of $D_{e} D_{e}^{\star}$ in $\mathbb{F}$;

(iv) the length of $\mathrm{P}_{e}$ is controlled by that of $\mathrm{P}$ in the following sense:

$$
\operatorname{len}\left(\left[\mathrm{P}_{e}\right]_{j, k}\right) \leqslant \max _{1 \leqslant n \leqslant r} \operatorname{len}\left([\mathrm{P}]_{n, k}\right) \quad \text { for all } 1 \leqslant j, k \leqslant s .
$$

We make some remarks about Theorem 2.1. The integer $J$ in item (iii) of Theorem 2.1 always satisfies $J \leqslant \operatorname{len}(\mathrm{P})$. Each of the elementary matrices $\mathrm{P}_{j}, j=$ $0, \ldots, J-1$ is a product of a constant matrix in $\mathbb{F}$ and a diagonal matrix whose diagonal elements are either 1 or $z^{-1}$. The matrix $\mathrm{P}_{J}$ is a product of a constant matrix in $\mathbb{F}$ and a diagonal matrix whose diagonal elements are monomials.

Theorem 2.1 shows that all the coefficients of the paraunitary extension matrix $\mathrm{P}_{e}$ are not "far away" from those of $\mathbb{F}$, provided that the given matrix $\mathrm{P}$ has the special form as in (2.1). In particular, when $\mathbb{F}$ is an algebraic number field $\mathbb{A}$, the extension matrix $\mathrm{P}_{e}$ is of the form $\mathrm{P}_{e}=D_{e} \mathrm{Q}_{e} D_{0}$ with $\mathrm{Q}_{e}$ being a matrix of Laurent polynomials with coefficients in $\mathbb{A}$. Consequently, all the coefficients of $\mathrm{P}_{e}$ are from an algebraic number field $\tilde{\mathbb{A}}$, where $\tilde{\mathbb{A}}$ is the field extension over $\mathbb{A}$ by adding the entries of $D_{0}$ and $D_{e}$. For the result on matrix extension with symmetry constraint over a general subfield $\mathbb{F}$; see Theorem 4.1 in Section 4 and also see Examples 6.1 - 6.4 in Section 6 for its application.

For row vectors $\mathrm{u}$ and $\mathrm{v}$ in $\mathbb{C}^{1 \times r}$, we define $\mathrm{v}^{\star}:=\overline{\mathrm{v}}^{\top},\langle\mathrm{u}, \mathrm{v}\rangle:=\mathrm{uv}^{\star}$, and $\|\mathrm{v}\|:=$ $\sqrt{\langle\mathrm{v}, \mathrm{v}\rangle}$. To prove Theorem 2.1 we need the following auxiliary result.

Lemma 2.2. Let $\mathrm{f}=\mathrm{g} D_{0}$ be a $1 \times s$ nonzero row vector for some $1 \times s$ row vector $\mathrm{g}$ with all the entries in $\mathbb{F}$ and some nonsingular diagonal matrix $D_{0}$ with all the entries of $D_{0} D_{0}^{\star}$ in $\mathbb{F}$. Then there exists an $s \times s$ unitary matrix $U_{\mathrm{f}}$ of the form $U_{\mathrm{f}}=D_{0}^{\star} V_{\mathrm{f}} D_{1}$ such that $U_{\mathrm{f}} U_{\mathrm{f}}^{\star}=I_{s}$ and $\mathrm{f} U_{\mathrm{f}}=[\|\mathrm{f}\|, 0, \ldots, 0]^{\top}$, where $V_{\mathrm{f}}$ is a nonsingular matrix with all the entries in $\mathbb{F}$, and where $D_{1}$ is some diagonal matrix with all the entries of $D_{1} D_{1}^{\star}$ in $\mathbb{F}$.

Proof. Let $\tilde{\mathrm{g}}_{1}:=\mathrm{g}$. Since $\tilde{\mathrm{g}}_{1} \neq \mathbf{0}$, we can find $1 \times s$ vectors $\mathrm{g}_{2}, \ldots, \mathrm{g}_{s}$ in $\mathbb{F}^{1 \times s}$ such that $\tilde{\mathrm{g}}_{1}, \mathrm{~g}_{2}, \ldots, \mathrm{g}_{s}$ are linearly independent. For instance, assuming $\mathrm{g}=$ $\left[g_{11}, \ldots, g_{1 s}\right]$ with $g_{11} \neq 0$, we can choose $\mathbf{g}_{j}=\mathbf{e}_{j}=[0, \ldots, 1, \ldots, 0]$, the standard $j$ th unit coordinate vector in $\mathbb{F}^{1 \times s}$, for $j=2, \ldots, s$. Then $\tilde{\mathrm{g}}_{1}, \mathrm{~g}_{2}, \ldots, \mathrm{g}_{s}$ are linearly independent. Because $D_{0}$ is nonsingular, all the vectors $\tilde{\mathrm{g}}_{1} D_{0}, \mathrm{~g}_{2} D_{0}, \ldots, \mathrm{g}_{s} D_{0}$ are linearly independent. Applying the Gram-Schmidt orthogonalization process to the linearly independent vectors $\tilde{\mathrm{g}}_{1} D_{0}, \mathrm{~g}_{2} D_{0}, \ldots, \mathrm{g}_{s} D_{0}$ as follows:

$$
\begin{aligned}
\mathrm{v}_{1} & :=\mathrm{g} D_{0}=\tilde{\mathrm{g}}_{1} D_{0}, \\
\mathrm{v}_{2} & :=\mathrm{g}_{2} D_{0}-\frac{\left\langle\mathrm{g}_{2} D_{0}, \tilde{\mathrm{g}}_{1} D_{0}\right\rangle}{\left\langle\tilde{\mathrm{g}}_{1} D_{0}, \tilde{\mathrm{g}}_{1} D_{0}\right\rangle} \tilde{\mathrm{g}}_{1} D_{0}=\left(\mathrm{g}_{2}-c_{2,1} \tilde{\mathrm{g}}_{1}\right) D_{0}=: \tilde{\mathrm{g}}_{2} D_{0}, \\
& \vdots \\
\mathrm{v}_{s} & :=\mathrm{g}_{s} D_{0}-\sum_{j=1}^{s-1} \frac{\left\langle\mathrm{g}_{s} D_{0}, \tilde{\mathrm{g}}_{j} D_{0}\right\rangle}{\left\langle\tilde{\mathrm{g}}_{j} D_{0}, \tilde{\mathrm{g}}_{j} D_{0}\right\rangle} \tilde{\mathrm{g}}_{j} D_{0}=\left(\mathrm{g}_{s}-c_{s, 1} \tilde{\mathrm{g}}_{1}-\cdots-c_{s, s-1} \tilde{\mathrm{g}}_{s-1}\right) D_{0}=: \tilde{\mathrm{g}}_{s} D_{0},
\end{aligned}
$$


where

and

$$
c_{k, j}:=\frac{\left\langle\mathrm{g}_{k} D_{0}, \tilde{\mathrm{g}}_{j} D_{0}\right\rangle}{\left\langle\tilde{\mathrm{g}}_{j} D_{0}, \tilde{\mathrm{g}}_{j} D_{0}\right\rangle}=\frac{\left\langle\mathrm{g}_{k} D_{0} D_{0}^{\star}, \tilde{\mathrm{g}}_{j}\right\rangle}{\left\langle\tilde{\mathrm{g}}_{j} D_{0} D_{0}^{\star}, \tilde{\mathrm{g}}_{j}\right\rangle}, \quad 1 \leqslant j<k \leqslant s
$$

$$
\tilde{\mathrm{g}}_{k}:=\mathrm{g}_{k}-c_{k, 1} \tilde{\mathrm{g}}_{1}-\cdots-c_{k, k-1} \tilde{\mathrm{g}}_{k-1}, \quad 1 \leqslant k \leqslant s .
$$

By our assumption, it is not difficult to verify that all $c_{k, j}$ and all entries of $\tilde{\mathrm{g}}_{k}$ are in $\mathbb{F}$ for all $1 \leqslant j<k \leqslant s$. It is also easy to check that $\left\langle\mathrm{v}_{j}, \mathrm{v}_{k}\right\rangle=\delta(j-k)\left\|\mathrm{v}_{j}\right\|^{2}$ for all $j, k=1, \ldots, s$, where $\delta$ denotes the Dirac sequence such that $\delta(0)=1$ and $\delta(k)=0$ for $k \neq 0$.

Finally, define $V_{\mathrm{f}}:=\left[\tilde{\mathrm{g}}_{1}^{\star}, \ldots, \tilde{\mathrm{g}}_{s}^{\star}\right]$ and $D_{1}:=\operatorname{diag}\left(\frac{\zeta_{1}}{\left\|\mathrm{v}_{1}\right\|}, \ldots, \frac{\zeta_{s}}{\left\|\mathrm{v}_{s}\right\|}\right)$ for any $\zeta_{1}, \ldots, \zeta_{s} \in$ $\mathbb{T}:=\{\zeta \in \mathbb{C}|| \zeta \mid=1\}$. It is straightforward to verify that $U_{\mathrm{f}}:=D_{0}^{\star} V_{\mathrm{f}} D_{1}$ is the desired unitary matrix.

Setting $\zeta_{1}=\cdots=\zeta_{s}=1$ in the above proof, we see that $D_{1}=\operatorname{diag}\left(\frac{1}{\left\|\mathrm{v}_{1}\right\|}, \ldots, \frac{1}{\left\|\mathrm{v}_{s}\right\|}\right)$. Define $\mathbb{F}_{+}^{1 / 2}:=\{\sqrt{x} \mid x \in \mathbb{F}, x \geqslant 0\}$. Then all $D_{1}$ 's constructed by employing Lemma 2.2 in this paper can be chosen in such a way that all entries of $D_{1}$ 's are from $\mathbb{F}_{+}^{1 / 2}$.

The role of $U_{\mathrm{f}}$ is to normalize the vector $\mathrm{f}$ by reducing $\mathrm{f}$ into a vector having only one nonzero entry. Note that $U_{\mathrm{f}}$ does not affect the zero entries of $f$ and there exists a permutation matrix $E$ such that

$$
\left[U_{\mathrm{f}} E\right]_{j,:}=\left(\left[U_{\mathrm{f}} E\right]_{:, j}\right)^{\top}=\mathbf{e}_{j}, \quad \text { provided }[\mathbf{f}]_{j}=0 .
$$

Here, $[A]_{j,:},[A]_{:, j}$ denote the $j$ th row, $j$ th column of a matrix $A$, respectively, and $[\mathrm{f}]_{j}$ is the $j$ th entry of a vector $\mathrm{f}$. For simplicity, we also define $U_{\mathrm{f}}=I_{s}$ for $\mathbf{f}=\mathbf{0}$ and $U_{\mathrm{f}}=\emptyset$ for $\mathrm{f}=\emptyset$, where $\emptyset$ is the emptyset. Throughout the paper, up to $a$ permutation means there exists a permutation matrix $E$ such that the identity holds exactly with the corresponding matrix $A$ being replaced by $A E$ (e.g., see (2.3)).

As a direct consequence of Lemma 2.2. for a general $r \times s$ constant matrix $F$ taking the form $F=G D_{0}$, we can construct a unitary matrix $U_{F}=D_{0}^{\star} V_{F} D_{1}$ so that $F U_{F}=[R, \mathbf{0}]$ and up to a permutation,

$$
\left[U_{F}\right]_{j,:}=\left(\left[U_{F}\right]_{:, j}\right)^{\top}=\mathbf{e}_{j}, \quad \text { provided }[F]_{:, j}=\mathbf{0},
$$

which can be summarized as the following corollary.

Corollary 2.3. Let $F=G D_{0}$ be an $r \times s$ matrix, where $G$ is an $r \times s$ matrix with all the entries in $\mathbb{F}$ and $D_{0}$ is a nonsingular diagonal matrix with all the entries of $D_{0} D_{0}^{\star}$ in $\mathbb{F}$. Then there exists an $s \times s$ unitary matrix $U_{F}$ of the form $U_{F}=D_{0}^{\star} V_{F} D_{1}$ such that $U_{F} U_{F}^{\star}=I_{s}$ and $F U_{F}=[R, 0]$ for some lower triangular matrix $R$, where $V_{F}$ is a nonsingular matrix with all the entries of $V_{F}$ in $\mathbb{F}$ and $D_{1}$ is some diagonal matrix with all the entries of $D_{1} D_{1}^{\star}$ in $\mathbb{F}$.

Proof. The main idea is to apply Lemma 2.2 to $F$ row by row.

Let $\mathrm{f}_{1}:=[F]_{1,:}=[G]_{1,:} D_{0}$ be the first row of $F$. By Lemma 2.2 , we can construct a unitary matrix $U_{1}=D_{0}^{\star} V_{1} D_{r}$ satisfying $\mathbf{f}_{1} U_{1}=\left[\left\|\mathbf{f}_{1}\right\|, 0, \ldots, 0\right]$ for some nonsingular $s \times s$ matrix $V_{1} \in \mathbb{F}^{s \times s}$ and some diagonal matrix $D_{r}$ with all the entries of $D_{r} D_{r}^{\star}$ in $\mathbb{F}$.

Next, let $F_{1}=F U_{1}$. Then $F_{1}=G_{1} D_{r}$ with $G_{1}=G D_{0} D_{0}^{\star} V_{1} \in \mathbb{F}^{r \times s}$. Let $\mathrm{f}_{2}:=\left[F_{1}\right]_{2,:}=\left[G_{1}\right]_{2,:} D_{r}$ be the second row of $F_{1}$. Applying Lemma 2.2 to $\tilde{\mathrm{f}}_{2}:=$ $\left[\mathrm{f}_{2}\right]_{2: s}$, the second row of $F_{1}$ ignoring the first entry of $\mathrm{f}_{2}$, we can, through a simple extension, find an $s \times s$ unitary matrix $U_{2}=D_{r}^{\star} V_{2} D_{r-1}$ satisfying $\mathrm{f}_{2} U_{2}=$ 
$\left[\left[\mathrm{f}_{2}\right]_{1},\left\|\tilde{\mathbf{f}}_{2}\right\|, 0, \ldots, 0\right]$ for some nonsingular $s \times s$ matrix $V_{2} \in \mathbb{F}^{s \times s}$ and some diagonal matrix $D_{r-1}$ with all the entries of $D_{r-1} D_{r-1}^{\star}$ in $\mathbb{F}$.

Repeating the above procedure, we can construct a sequence of $s \times s$ unitary matrices $U_{1}=D_{0}^{\star} V_{1} D_{r}, U_{2}=D_{r}^{\star} V_{2} D_{r-1}, \ldots, U_{r}=D_{2}^{\star} V_{r} D_{1}$ by Lemma 2.2 . which reduce the matrix $F$ to a lower triangular matrix. That is $F U_{1} \cdots U_{r}=$ $[R, \mathbf{0}]$ for some lower triangular matrix $R$. Let $U_{F}:=D_{0}^{\star} V_{F} D_{1}$ with $V_{F}:=$ $V_{1} D_{r} D_{r}^{\star} \cdots V_{r-1} D_{2} D_{2}^{\star} V_{r}$. Then $U_{F}$ is a desired matrix.

Let $\mathrm{p}$ be a $1 \times s$ vector of Laurent polynomials taking the form $\mathrm{p}(z)=\mathrm{q}_{0}(z) D_{0}$. Suppose $\operatorname{fsupp}(\mathrm{p})=[\ell, h]$ with $h-\ell>0$. Then $\mathrm{p}$ can be written as

$$
\mathrm{p}(z)=\mathrm{f}_{\ell} z^{\ell}+\cdots+\mathrm{f}_{h} z^{h}=\left(\mathrm{g}_{\ell} z^{\ell}+\cdots+\mathrm{g}_{h} z^{h}\right) D_{0}, \quad z \in \mathbb{C} \backslash\{0\} .
$$

Suppose that $\mathrm{p}$ is paraunitary, i.e., $\mathrm{p}(z) \mathrm{p}^{\star}(z)=1$ for all $z \in \mathbb{C} \backslash\{0\}$. For the row vector $\mathrm{f}_{\ell}$, we can construct a unitary matrix $U_{\mathrm{f}_{\ell}}$ by Lemma 2.2 such that $U_{\mathrm{f}_{\ell}} U_{\mathbf{f}_{\ell}}^{\star}=$ $I_{s}$ and $\mathbf{f}_{\ell} U_{\mathbf{f}_{\ell}}=\left[\left\|\mathbf{f}_{\ell}\right\|, 0, \ldots, 0\right]$. Because $\mathrm{pp}^{\star}=1$, we have $\left\langle\mathbf{f}_{\ell} U_{\mathbf{f}_{\ell}}, \mathbf{f}_{h} U_{\mathbf{f}_{\ell}}\right\rangle=0$. Consequently, $\mathbf{f}_{h} U_{\mathbf{f}_{\ell}}$ must take the form $\mathbf{f}_{h} U_{\mathbf{f}_{\ell}}=[0, *, \ldots, *]$, where $*$ denotes some number in $\mathbb{F}$. Using a diagonal matrix $\mathrm{D}(z):=\operatorname{diag}\left(1, z^{-1}, \ldots, z^{-1}\right)$, we can reduce the length of $\mathrm{p}$ by 1 . Replacing $\mathrm{p}$ by $\mathrm{p} U_{\mathrm{f}_{\ell}} \mathrm{D}$ and repeating this procedure, we can reduce the length of $\mathrm{p}$ step by step to 0 . This is the main idea to prove Theorem 2.1 for the case $r=1$. The same idea, by employing Corollary 2.3 instead of Lemma 2.2, yields the following proof of Theorem 2.1.

Proof of Theorem 2.1. The sufficiency part is trivial. We next prove the necessity part. Suppose that $\mathrm{P}$ takes the form in (2.1) and $\mathrm{P}(z) \mathrm{P}^{\star}(z)=I_{r}$ for all $z \in \mathbb{C} \backslash\{0\}$. Assume that $\operatorname{fsupp}(\mathrm{P})=[\ell, h]$ with $h-\ell>0$. Then $\mathrm{P}=\mathrm{Q}_{0} D_{0}$ takes the form

$$
\mathrm{P}(z)=F_{\ell} z^{\ell}+\cdots+F_{h} z^{h}=\left(G_{\ell} z^{\ell}+\cdots+G_{h} z^{h}\right) D_{0}, \quad z \in \mathbb{C} \backslash\{0\},
$$

where $F_{j}, G_{j}$ for $j=\ell, \ldots, h$ are $r \times s$ constant matrices and $\mathrm{Q}_{0}(z)=G_{\ell} z^{\ell}+\cdots+$ $G_{h} z^{h}$. We now perform the following steps to reduce the length of $\mathrm{P}$ by at least 1.

(1) Since $F_{\ell}=G_{\ell} D_{0} \neq \mathbf{0}$, by Corollary 2.3, we can construct a unitary matrix $U_{F_{\ell}}$ of the form $U_{F_{\ell}}=D_{0}^{\star} V_{F_{\ell}} D_{1}$ such that $U_{F_{\ell}} U_{F_{\ell}}^{\star}=I_{s}$ and $F_{\ell} U_{F_{\ell}}=[R, \mathbf{0}]$, where $V_{F_{\ell}}$ is a nonsingular matrix with all the entries in $\mathbb{F}, D_{1}$ is a diagonal matrix with all the entries of $D_{1} D_{1}^{\star}$ in $\mathbb{F}$, and $R$ is an $r \times m$ lower triangular matrix with $m$ being the rank of $F_{\ell}$.

(2) In view of the paraunitary property, $\mathrm{P}(z) \mathrm{P}^{\star}(z)=I_{r}$ for all $z \in \mathbb{C} \backslash\{0\}$ deduces that $\left(F_{\ell} U_{F_{\ell}}\right)\left(F_{h} U_{F_{\ell}}\right)^{\star}=\mathbf{0}$. Hence, $\mathrm{P} U_{F_{\ell}}$ must take the following form:

$$
\mathrm{P}(z) U_{F_{\ell}}=\left[R, \mathbf{0}_{\tilde{R}}\right] z^{\ell}+\cdots+\left[\mathbf{0}_{R}, \tilde{R}\right] z^{h}, \quad z \in \mathbb{C} \backslash\{0\},
$$

where $\mathbf{0}_{R}$ is the $r \times m$ zero matrix having the same size as $R$.

(3) Define $\mathrm{D}_{0}(z):=\operatorname{diag}\left(\mathbf{1}_{m}, z^{-1} \mathbf{1}_{s-m}\right)$ and $\tilde{\mathrm{P}}(z):=\mathrm{P}(z) U_{F_{\ell}} \mathrm{D}_{0}(z)$. Here $\mathbf{1}_{m}$ denotes the $1 \times m$ row vector $[1, \ldots, 1]$. Then $\tilde{\mathrm{P}}(z)=\mathrm{Q}_{1}(z) D_{1}$, with $\mathrm{Q}_{1}(z):=\mathrm{Q}_{0}(z) D_{0} D_{0}^{\star} V_{F_{\ell}} \mathrm{D}_{0}(z)$ being an $r \times s$ matrix of Laurent polynomials in $\mathbb{F}\left[z, z^{-1}\right]$, satisfies fupp $(\tilde{\mathrm{P}})=[\ell, h-1]$. Define $\mathrm{A}_{0}(z):=U_{F_{\ell}} \mathrm{D}_{0}(z)=$ $D_{0}^{\star} \mathrm{V}_{0}(z) D_{1}$ with $\mathrm{V}_{0}(z):=V_{F_{\ell}} \mathrm{D}_{0}(z)$. Then $\mathrm{A}_{0}(z)$ is paraunitary.

Repeating the above steps, we can construct a sequence of paraunitary matrices $\mathrm{A}_{j}(z)=D_{j}^{\star} \mathrm{V}_{j}(z) D_{j+1}, j=0, \ldots, J$ such that $\mathrm{PA}_{0} \cdots \mathrm{A}_{J}=\left[I_{r}, \mathbf{0}\right]$. Define $\mathrm{P}_{e}:=$ $\mathrm{A}_{J}^{\star} \cdots \mathrm{A}_{0}^{\star}$. Then it is easy to show that $\mathrm{P}_{e}$ takes the form as in Item (iii). Items (i) - (iii) follow directly from the above construction. Item (iv) follows from the property of $U_{F_{\ell}}$ in (2.3). 
Based on the constructive proof of Theorem 2.1 we provide an algorithm (see Algorithm (1) for the matrix extension problem without symmetry constraint. In the algorithm and this paper, for an $r \times s$ matrix $\mathrm{P}(z)=\sum_{k \in \mathbb{Z}} P_{k} z^{k}$ of Laurent polynomials, coeff $(\mathrm{P}, k)$ refers to the coefficient matrix $P_{k}$ of $z^{k}$.

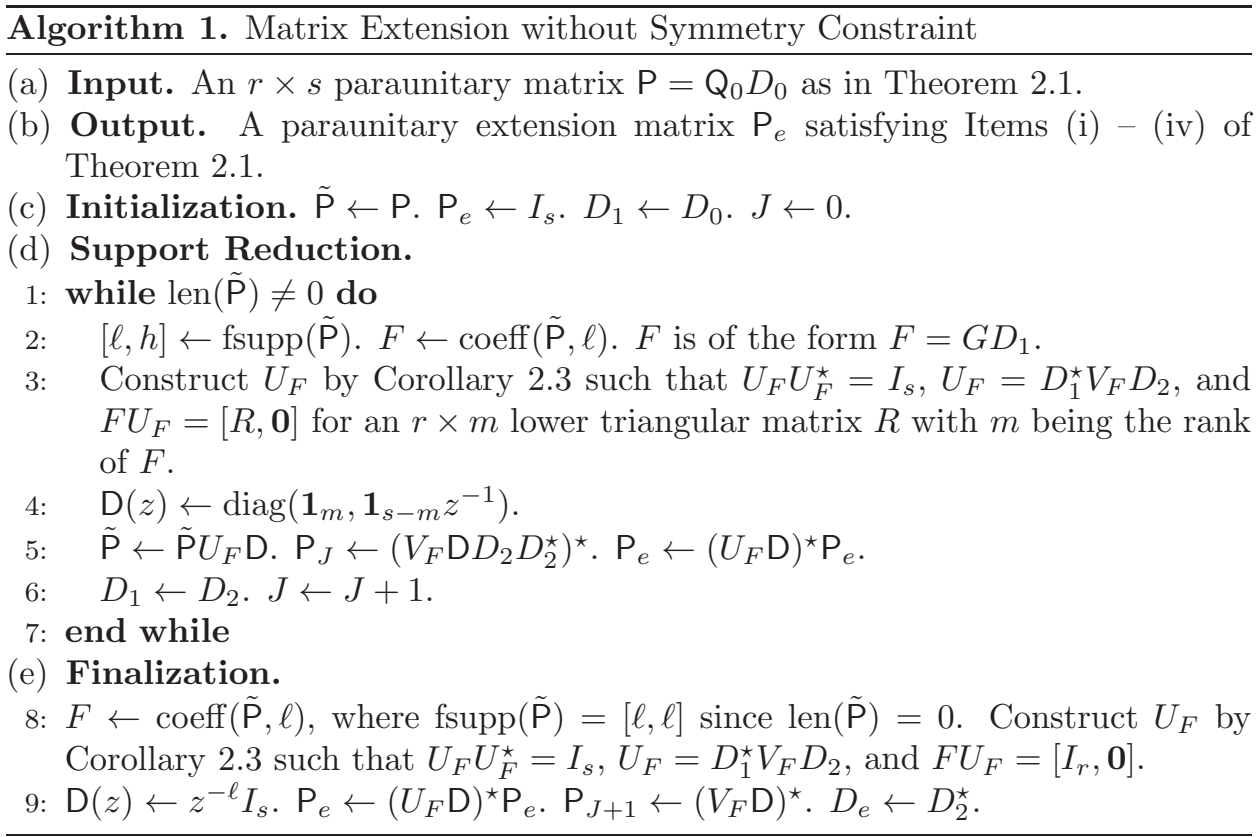

\section{Application to orthogonal WaVelet filter Banks}

In this section, we shall discuss the application of our result on the matrix extension problem without symmetry in Section 2 to d-orthogonal wavelet filter banks in electronic engineering and wavelet analysis. We shall also explain in this section why our formulation of the matrix extension problem over a general field leads to a satisfactory solution to the construction of orthogonal wavelet filter banks over algebraic number fields.

Let us first recall some definitions and notation. We say that $\mathrm{d}$ is a dilation factor if $d$ is an integer with $|d|>1$. For simplicity of presentation, we further assume that $\mathrm{d}$ is positive, while the case of a negative dilation factor can be handled similarly by a slight modification of the statements in this paper.

Recall that in this paper $\mathbb{F}$ always denotes a general subfield of $\mathbb{C}$ satisfying (1.1). A filter $a=\{a(k)\}_{k \in \mathbb{Z}}: \mathbb{Z} \rightarrow \mathbb{F}^{r \times r}$ with multiplicity $r$ is a finitely supported sequence of $r \times r$ matrices on $\mathbb{Z}$. The $z$-transform or symbol of the filter $a$ is defined to be

$$
\mathrm{a}(z):=\sum_{k \in \mathbb{Z}} a(k) z^{k}, \quad z \in \mathbb{C} \backslash\{0\},
$$

which is a matrix of Laurent polynomials with coefficients in $\mathbb{F}^{r \times r}$. Moreover, the polyphase components of a (or a) are defined by

$$
\mathrm{a}^{[\gamma]}(z):=\sum_{k \in \mathbb{Z}} a(\gamma+\mathrm{d} k) z^{k}, \quad \gamma \in \mathbb{Z} .
$$


We say that a (or $a$ ) is a d-orthogonal wavelet filter if

$$
\sum_{\gamma=0}^{\mathrm{d}-1} \mathrm{a}^{[\gamma]}(z) \mathrm{a}^{[\gamma]}(z)^{\star}=\mathrm{d}^{-1} I_{r}, \quad z \in \mathbb{C} \backslash\{0\} .
$$

Let $b_{1}, \ldots, b_{\mathrm{d}-1}: \mathbb{Z} \rightarrow \mathbb{C}^{r \times r}$ be filters with multiplicity $r$. We say that the set of filters $\left\{a ; b_{1}, \ldots, b_{\mathrm{d}-1}\right\}$ is a d-orthogonal wavelet filter bank if the following polyphase matrix

$$
\mathcal{P}(z)=\sqrt{\mathrm{d}}\left[\begin{array}{ccc}
\mathrm{a}^{[0]}(z) & \cdots & \mathrm{a}^{[\mathrm{d}-1]}(z) \\
\mathrm{b}_{1}^{[0]}(z) & \cdots & \mathrm{b}_{1}^{[\mathrm{d}-1]}(z) \\
\vdots & \ddots & \vdots \\
\mathrm{b}_{\mathrm{d}-1}^{[0]}(z) & \cdots & \mathrm{b}_{\mathrm{d}-1}^{[\mathrm{d}-1]}(z)
\end{array}\right], \quad z \in \mathbb{C} \backslash\{0\}
$$

is paraunitary; that is, $\mathcal{P}(z) \mathcal{P}^{\star}(z)=I_{\mathrm{d} r}$ for all $z \in \mathbb{C} \backslash\{0\}$, where each $\mathbf{b}_{m}^{[\gamma]}$ is a polyphase component of $b_{m}$ defined similarly as in (3.1) for $m, \gamma=0, \ldots, d-1$, respectively. Without any a priori condition, any wavelet filter bank naturally corresponds to a frequency-based framelet in the distribution space; see Han [7] for more detail.

There are two major tasks in the construction of orthogonal wavelet filter banks. One is to construct a d-orthogonal wavelet low-pass filter $a$ with some desirable properties, and the other is to derive the associated high-pass filters $b_{1}, \ldots, b_{\mathrm{d}-1}$ from a given $\mathrm{d}$-orthogonal wavelet low-pass filter $a$ so that $\left\{a ; b_{1}, \ldots, b_{\mathrm{d}-1}\right\}$ is a d-orthogonal wavelet filter bank.

The matrix extension problem plays a major role in the second part for deriving the high-pass filters $b_{1}, \ldots, b_{\mathrm{d}-1}$ from a given $\mathrm{d}$-orthogonal wavelet low-pass filter $a$. As an application of Theorem 2.1. we can construct the high-pass filters $b_{1}, \ldots, b_{\mathrm{d}-1}$ systematically when the d-orthogonal wavelet filter $a$ is given. In fact, let $\mathrm{P}:=$ $\sqrt{\mathrm{d}}\left[\mathrm{a}^{[0]}, \ldots, \mathrm{a}^{[\mathrm{d}-1]}\right]=: \sqrt{\mathrm{d}} Q_{0}$. Then $\mathrm{P}=\mathrm{Q}_{0} D_{0}$, with $D_{0}:=\sqrt{\mathrm{d}} I_{s}$, satisfies the conditions in Theorem 2.1. Thus, we can extend $\mathrm{P}$ to a full paraunitary matrix $\mathrm{P}_{e}(z)=: \mathcal{P}(z)$ as in (3.3), from which it is not difficult to construct high-pass filters $b_{1}, \ldots, b_{\mathrm{d}-1}$; see (5.11). More importantly, Theorem 2.1 guarantees that the coefficients of the high-pass filters are not "far away" from the field generated by the coefficients of the low-pass filter. This is summarized in Theorem 3.1 as follows. For its proof, see the proof of the more detailed version in Theorem 5.2 of Section 5 .

Theorem 3.1. Let $a: \mathbb{Z} \rightarrow \mathbb{F}^{r \times r}$ be a d-orthogonal wavelet low-pass filter with multiplicity $r$. Then there exist high-pass filters $b_{1}, \ldots, b_{\mathrm{d}-1}: \mathbb{Z} \rightarrow \mathbb{C}^{r \times r}$ such that $\left\{a ; b_{1}, \ldots, b_{\mathrm{d}-1}\right\}$ forms a d-orthogonal wavelet filter bank and $b_{m}=D_{m} \tilde{b}_{m}$ for $m=1, \ldots, \mathrm{d}-1$, where $\tilde{b}_{m}: \mathbb{Z} \rightarrow \mathbb{F}^{r \times r}$ has coefficients in $\mathbb{F}$ and $D_{m}$ is some diagonal matrix with all the entries of $D_{m} D_{m}^{\star}$ in $\mathbb{F}$.

Algorithm 3 in Section 5 provides the detail for the construction of the highpass filters $b_{1}, \ldots, b_{\mathrm{d}-1}$ in Theorem 3.1 and see Examples 6.1-6.4 in Section 6 for the illustration. In the rest of this section we shall explain why almost all finitely supported $d$-orthogonal wavelet filters have coefficients from an algebraic number field, and why our formulation of the matrix extension problem over a general field is satisfactory.

Assume that we are looking for a d-orthogonal wavelet filter $a$ with multiplicity $r$ such that $\operatorname{fsupp}(a) \subseteq[m, n]$. Regard the entries in the matrices $a(k), k=m, \ldots, n$ as 
unknowns. Then the orthogonality condition in (3.2) induces a system of quadratic equations with rational coefficients. Finding a Gröbner basis of the system and setting the unconstrained unknowns in the Gröbner basis to be numbers from an algebraic number field (or simply from the rational number field), we see that all the unknowns are roots of polynomials with coefficients from some algebraic number fields. Consequently, by solving a system of quadratic equations with rational coefficients induced by (3.2), all the coefficients of a d-orthogonal wavelet filter are from certain algebraic number field. This more or less explains why all finitely supported d-orthogonal wavelet filters known in the literature have their coefficients from certain algebraic number field. See [1, 2, 3, 4, 5, 6, 8, 12, 13, 18, and Section 6 for many such examples. In particular, [8] provides a systematic construction for d-orthogonal wavelet low-pass filters with linear-phase moments and/or symmetry.

Now we assume that $a$ is a finitely supported d-orthogonal wavelet filter such that all its coefficients are from an algebraic number field $\mathbb{A}$. It is quite appealing and desirable that one can derive a d-orthogonal wavelet filter bank $\left\{a ; b_{1}, \ldots, b_{\mathrm{d}-1}\right\}$ such that all the coefficients of $b_{1}, \ldots, b_{\mathrm{d}-1}$ are also from the same algebraic number field $\mathbb{A}$. However, as demonstrated by many examples available in the literature and obtained by brute force calculation using Gröbner bases in symbolic computation, such a requirement is too strong or ideal to be feasible. For the convenience of the reader, we provide a simple example here to illustrate this.

Example 3.2. Let $\mathrm{d}=3$ and $a$ be given by $\mathrm{a}(z)=\frac{1}{3}\left(1+z+z^{2}\right)$. Then all the coefficients of $a$ are in the rational number field $\mathbb{Q}$, and it is easy to show that $a$ is 3 -orthogonal. Suppose that $b_{1}$ and $b_{2}$ are two high-pass filters such that $\left\{a ; b_{1}, b_{2}\right\}$ forms a 3-orthogonal wavelet filter bank. It is very much desired in both theory and application that $\max \left(\operatorname{len}\left(b_{1}\right), \operatorname{len}\left(b_{2}\right)\right) \leqslant \operatorname{len}(a)=2$ (most d-orthogonal wavelet filter banks in the literature satisfy this property). In our case, we wish to construct high-pass filters $b_{1}$ and $b_{2}$ with coefficients in $\mathbb{Q}$ and with length no more than 2 . In terms of polyphase components, this is equivalent to requiring that the polyphase matrix should take the form

$$
\mathcal{P}(z)=\sqrt{\mathrm{d}}\left[\begin{array}{lll}
\mathrm{a}^{[0]}(z) & \mathrm{a}^{[1]}(z) & \mathrm{a}^{[2]}(z) \\
\mathrm{b}_{1}^{[0]}(z) & \mathrm{b}_{1}^{[1]}(z) & \mathrm{b}_{1}^{[2]}(z) \\
\mathrm{b}_{2}^{[0]}(z) & \mathrm{b}_{2}^{[1]}(z) & \mathrm{b}_{2}^{[2]}(z)
\end{array}\right]=\sqrt{3}\left[\begin{array}{ccc}
\frac{1}{3} & \frac{1}{3} & \frac{1}{3} \\
t_{1} z^{m} & t_{2} z^{m} & t_{3} z^{m} \\
s_{1} z^{n} & s_{2} z^{n} & s_{3} z^{n}
\end{array}\right],
$$

where $t_{1}, t_{2}, t_{3}, s_{1}, s_{2}, s_{3} \in \mathbb{Q}$ and $m, n \in \mathbb{Z}$. It is straightforward to see that the orthogonality condition $\mathcal{P}(z) \mathcal{P}^{\star}(z)=I_{3}$ for all $z \in \mathbb{C} \backslash\{0\}$ implies

$$
t_{1}+t_{2}+t_{3}=0, \quad t_{1}^{2}+t_{2}^{2}+t_{3}^{2}=\frac{1}{3} .
$$

So, we need to find a rational solution $\left\{t_{1}, t_{2}, t_{3}\right\}$ to the system of equations in (3.4). Solving (3.4), we have $t_{1}=x, t_{2}=-\frac{x}{2} \pm \frac{\sqrt{6-27 x^{2}}}{6}$, and $t_{3}=-\frac{x}{2} \mp \frac{\sqrt{6-27 x^{2}}}{6}$, where $x$ is a free parameter. To have a solution such that $t_{1}, t_{2}, t_{3} \in \mathbb{Q}$, we must require $x \in \mathbb{Q}$ and $\sqrt{6-27 x^{2}} \in \mathbb{Q}$. Equivalently, we need to find a rational point $(x, y) \in \mathbb{Q}^{2}$ satisfying the equation $27 x^{2}+y^{2}-6=0$, which defines an algebraic curve. Finding a rational point on an algebraic curve is a major task in computational algebraic geometry. In other words, our question becomes whether or not the algebraic curve $27 x^{2}+y^{2}-6=0$ on $\mathbb{R}^{2}$ contains a rational point $(x, y) \in \mathbb{Q}^{2}$. Using the package algcurves in maple, we find that the algebraic curve $27 x^{2}+y^{2}-6=0$ has genus 0 and is irreducible. Therefore, the algebraic curve has a parametrization. Using parametrization in the software maple for symbolic computation, from 
the parametrization of the algebraic curve $27 x^{2}+y^{2}-6=0$, we conclude that the algebraic curve does not contain any rational point $(x, y) \in \mathbb{Q}^{2}$. This shows that it is impossible to have a 3 -orthogonal wavelet filter bank $\left\{a ; b_{1}, b_{2}\right\}$ such that $\max \left(\operatorname{len}\left(b_{1}\right), \operatorname{len}\left(b_{2}\right)\right) \leqslant \operatorname{len}(a)$ and $b_{1}, b_{2}$ have rational coefficients. In fact, the above argument shows that if $b$ is a 3-orthogonal high-pass filter to $a$ and $b$ has rational coefficients, then we must have $\operatorname{len}(b) \geqslant 3>\operatorname{len}(a)$.

On the other hand, if we relax the "rational coefficients" condition to "rational coefficients up to a multiplicative constant", we have the following solution: $m=n=0, t_{1}=\frac{\sqrt{6}}{6}, t_{2}=0, t_{3}=-\frac{\sqrt{6}}{6}, s_{1}=\frac{\sqrt{2}}{6}, s_{2}=-\frac{\sqrt{2}}{3}, s_{3}=\frac{\sqrt{2}}{6}$. The corresponding high-pass filters are given by

$$
\mathrm{b}_{1}(z)=\frac{\sqrt{6}}{6}\left(1-z^{2}\right), \quad \mathrm{b}_{2}(z)=\frac{\sqrt{2}}{6}\left(1-2 z+z^{2}\right),
$$

each of which has coefficients in $\mathbb{Q}$ up to a multiplicative constant and len $\left(b_{1}\right)=$ $\operatorname{len}\left(b_{2}\right)=\operatorname{len}(a)$.

To appreciate our results and our formulation of the matrix extension problem, in the following let us look at the multilevel wavelet transform. To do so, let us introduce some notation and definitions.

The multilevel wavelet transform is implemented by using the subdivision operator for reconstruction and the transition operator for decomposition. For a filter $a: \mathbb{Z} \rightarrow \mathbb{F}^{r \times r}$ with multiplicity $r$, the subdivision operator $\mathcal{S}_{a, \mathrm{~d}}:\left(l_{2}(\mathbb{Z})\right)^{1 \times r} \rightarrow$ $\left(l_{2}(\mathbb{Z})\right)^{1 \times r}$ is defined to be

$$
\left[\mathcal{S}_{a, \mathrm{~d}} v\right](n):=\mathrm{d} \sum_{k \in \mathbb{Z}} v(k) a(n-\mathrm{d} k), \quad n \in \mathbb{Z}, v \in\left(l_{2}(\mathbb{Z})\right)^{1 \times r}
$$

and the transition operator $\mathcal{T}_{a, \mathrm{~d}}:\left(l_{2}(\mathbb{Z})\right)^{1 \times r} \rightarrow\left(l_{2}(\mathbb{Z})\right)^{1 \times r}$ is defined to be

$$
\left[\mathcal{T}_{a, \mathrm{~d}} v\right](n):=\mathrm{d} \sum_{k \in \mathbb{Z}} v(k) \overline{a(k-\mathrm{d} n)}^{\top}, \quad n \in \mathbb{Z}, v \in\left(l_{2}(\mathbb{Z})\right)^{1 \times r} .
$$

For a positive integer $J$, the $J$-level discrete wavelet decomposition is given by

$$
v_{j-1}=\frac{\sqrt{\mathrm{d}}}{\mathrm{d}} \mathcal{T}_{a, \mathrm{~d}} v_{j} \quad \text { and } \quad w_{j-1 ; m}=\frac{\sqrt{\mathrm{d}}}{\mathrm{d}} \mathcal{T}_{b_{m}, \mathrm{~d}} v_{j}, \quad m=1, \ldots, \mathrm{d}-1 ; j=J, \ldots, 1,
$$

where $v_{J}: \mathbb{Z} \rightarrow \mathbb{C}^{1 \times r}$ is an input signal in $\left(l_{2}(\mathbb{Z})\right)^{1 \times r}$. Moreover, entries in $v_{0}$ are called low-pass wavelet coefficients and entries in $w_{j-1 ; m}$ are called high-pass wavelet coefficients.

The $J$-level discrete wavelet reconstruction is given by

$$
\stackrel{\circ}{v}_{j}=\frac{\sqrt{\mathrm{d}}}{\mathrm{d}} \mathcal{S}_{a, \mathrm{~d}} \stackrel{\circ}{v}_{j-1}+\frac{\sqrt{\mathrm{d}}}{\mathrm{d}} \sum_{m=1}^{\mathrm{d}-1} \mathcal{S}_{b_{m}, \mathrm{~d}} \stackrel{\circ}{w}_{j-1 ; m}, \quad j=1, \ldots, J .
$$

Suppose that $\left\{a ; b_{1}, \ldots, b_{\mathrm{d}-1}\right\}$ is a d-orthogonal wavelet filter bank. Then we can deduce from the identity $\mathcal{P}(z) \mathcal{P}^{\star}(z)=I_{r}$ for all $z \in \mathbb{C} \backslash\{0\}$ that

$$
\mathcal{S}_{a, \mathrm{~d}} \mathcal{T}_{a, \mathrm{~d}} v+\sum_{m=1}^{\mathrm{d}-1} \mathcal{S}_{b_{m}, \mathrm{~d}} \mathcal{T}_{b_{m}, \mathrm{~d}} v=\mathrm{d} v, \quad v \in\left(l_{2}(\mathbb{Z})\right)^{1 \times r}
$$

Noting that $\left\|\mathcal{T}_{b_{m}, \mathrm{~d}} v\right\|_{\left(l_{2}(\mathbb{Z})\right)^{1 \times r}}^{2}=\left\langle\mathcal{T}_{b_{m}, \mathrm{~d}} v, \mathcal{T}_{b_{m}, \mathrm{~d}} v\right\rangle=\left\langle\mathcal{S}_{b_{m}, \mathrm{~d}} \mathcal{T}_{b_{m}, \mathrm{~d}} v, v\right\rangle$, we now have the following energy preserving equality:

$$
\left\|v_{J}\right\|_{\left(l_{2}(\mathbb{Z})\right)^{1 \times r}}^{2}=\left\|v_{0}\right\|_{\left(l_{2}(\mathbb{Z})\right)^{1 \times r}}^{2}+\sum_{j=1}^{J} \sum_{m=1}^{\mathrm{d}-1}\left\|w_{j-1 ; m}\right\|_{\left(l_{2}(\mathbb{Z})\right)^{1 \times r}}^{2} .
$$


Moreover, if nothing is performed on the wavelet coefficients, i.e., $\stackrel{\circ}{v}_{0}=v_{0}$ and ${\stackrel{\circ}{w_{j-1 ; m}}}_{j}=w_{j-1 ; m}$ for all $m=1, \ldots, \mathrm{d}-1$ and $J=1, \ldots, J$, then we must have the perfect reconstruction property: $\stackrel{\circ}{v}_{J}=v_{J}$, that is, the original input signal $v_{J}$ can be exactly reconstructed.

We now explain that our formulation of the matrix extension problem over a general field leads to a satisfactory solution to the construction of orthogonal wavelet filter banks.

Suppose that $a: \mathbb{Z} \rightarrow \mathbb{A}^{r \times r}$ is a d-orthogonal wavelet filter with multiplicity $r$ such that all the coefficients of $a$ belong to an algebraic number field $\mathbb{A}$ (for example, $\mathbb{A}=\mathbb{Q}$ ). By Theorem 3.1 , we can construct a d-orthogonal wavelet filter bank $\left\{a ; b_{1}, \ldots, b_{\mathrm{d}-1}\right\}$ such that the polyphase matrix $\mathcal{P}$ in (3.3) is paraunitary and

$$
b_{m}=D_{m} \tilde{b}_{m}, \quad m=1, \ldots, \mathrm{d}-1,
$$

where all the filters $\tilde{b}_{m}: \mathbb{Z} \rightarrow \mathbb{A}^{r \times r}, m=1, \ldots, \mathrm{d}-1$ with multiplicity $r$ have their coefficients in $\mathbb{A}$, and all $D_{m}$ are $r \times r$ diagonal matrices with all the entries of $D_{m} D_{m}^{\star}$ in $\mathbb{A}$.

Let $\tilde{v}_{J}:=v_{J}$. We modify the $J$-level discrete wavelet decomposition as follows:

$$
\tilde{v}_{j-1}:=\mathcal{T}_{a, \mathrm{~d}} \tilde{v}_{j} \quad \text { and } \quad \tilde{w}_{j-1 ; m}:=\mathcal{T}_{\tilde{b}_{m}, \mathrm{~d}} \tilde{v}_{j}
$$

for $j=J, \ldots, 1$ and $m=1, \ldots, \mathrm{d}-1$. Then we have the following relations:

$$
v_{j-1}=\mathrm{d}^{(j-1-J) / 2} \tilde{v}_{j-1}, \quad w_{j-1 ; m}=\mathrm{d}^{(j-1-J) / 2} \tilde{w}_{j-1 ; m} D_{m}^{\star}
$$

for $m=1, \ldots, \mathrm{d}-1, j=1, \ldots, J$. That is, the original wavelet coefficients $v_{j-1}$ and $w_{j-1 ; m}$ are simply scaled versions of the new wavelet coefficients $\tilde{v}_{j-1}$ and $\tilde{w}_{j-1 ; m}$. Suppose that the input signal $v_{J}$ also has its entries from the algebraic number field $\mathbb{A}$ (in fact, the coefficients of $v_{J}$ are often rational numbers). Then all the new wavelet coefficients $\tilde{v}_{j-1}$ and $\tilde{w}_{j-1 ; m}$ can be efficiently computed using integer arithmetics and matrix/vector operations in linear algebra. Also, by the definition of the transition operator in (3.5) and the relation in (3.7), we see that the $J$-level discrete wavelet reconstruction can be modified by

$$
\stackrel{\check{v}}{j}_{j}=\mathrm{d}^{-1} \mathcal{S}_{a, \mathrm{~d}} \stackrel{\tilde{v}}{j-1}_{j}+\mathrm{d}^{-1} \sum_{m=1}^{\mathrm{d}-1} \mathcal{S}_{\tilde{b}_{m}, \mathrm{~d}}\left(\stackrel{\tilde{w}}{j-1 ; m}_{m}^{\star} D_{m}\right), \quad j=1, \ldots, J .
$$

Since all the entries of the diagonal matrices $D_{m}^{\star} D_{m}$ are from $\mathbb{A}$, the above modified $J$-level discrete wavelet reconstruction in (3.8) can be also efficiently implemented using integer arithmetics and matrix/vector operations in linear algebra. Moreover, if nothing is performed on the new wavelet coefficients, i.e., $\stackrel{\circ}{v}_{0}=\tilde{v}_{0}$ and $\stackrel{\circ}{\tilde{w}}_{j-1 ; m}=$ $\tilde{w}_{j-1 ; m}$ for all $m=1, \ldots, \mathrm{d}-1$ and $j=1, \ldots, J$, then we must have the perfect reconstruction property: $\stackrel{\stackrel{v}{v}}{J}_{J}=\tilde{v}_{J}=v_{J}$; that is, the original input signal $v_{J}$ can be exactly reconstructed by the modified discrete wavelet transform.

\section{THE MATRIX EXTENSION PROBLEM WITH SYMMETRY CONSTRAINT}

In this section, we shall investigate the matrix extension problem with symmetry constraint over a general subfield $\mathbb{F}$ of $\mathbb{C}$ satisfying (1.1). The matrix extension problem with symmetry constraint is much more challenging than its counterpart without the symmetry constraint in Section 2. Extra effort is needed to guarantee the symmetry structure of the extension matrix. Let us first recall some necessary notation and definitions related to symmetry of Laurent polynomials. 
Let $\mathrm{p}(z)=\sum_{k \in \mathbb{Z}} p_{k} z^{k}, z \in \mathbb{C} \backslash\{0\}$ be a Laurent polynomial with complex coefficients $p_{k} \in \mathbb{C}$. We say that $\mathrm{p}$ has symmetry if its coefficient sequence $\left\{p_{k}\right\}_{k \in \mathbb{Z}}$ has symmetry; more precisely, there exist $\varepsilon \in\{-1,1\}$ and $c \in \mathbb{Z}$ such that

$$
p_{c-k}=\varepsilon p_{k} \quad \forall k \in \mathbb{Z} .
$$

If $\varepsilon=1$, then $\mathrm{p}$ is symmetric about the point $c / 2$; if $\varepsilon=-1$, then $\mathrm{p}$ is antisymmetric about the point $c / 2$. Symmetry of a Laurent polynomial can be conveniently expressed using a symmetry operator $\mathrm{S}$ defined by

$$
\operatorname{Sp}(z):=\frac{\mathrm{p}(z)}{\mathrm{p}\left(z^{-1}\right)}, \quad z \in \mathbb{C} \backslash\{0\} .
$$

When $\mathrm{p}$ is not identically zero, it is evident that (4.1) holds if and only if $\operatorname{Sp}(z)=$ $\varepsilon z^{c}$. For the zero polynomial, it is very natural that S0 can be assigned any symmetry pattern; that is, for every occurrence of S0 appearing in an identity in this paper, S0 is understood to take an appropriate choice of $\varepsilon z^{c}$ for some $\varepsilon \in\{-1,1\}$ and $c \in \mathbb{Z}$ so that the identity holds. If $\mathrm{P}$ is an $r \times s$ matrix of Laurent polynomials with symmetry, then we can apply the operator $\mathrm{S}$ to each entry of $\mathrm{P}$; that is, SP is an $r \times s$ matrix such that $[\mathrm{SP}]_{j, k}:=\mathrm{S}\left([\mathrm{P}]_{j, k}\right)$ for $1 \leqslant j \leqslant r$ and $1 \leqslant k \leqslant s$.

For two matrices $\mathrm{P}$ and $\mathrm{Q}$ of Laurent polynomials with symmetry, even though all the entries in $P$ and $Q$ have symmetry, their sum $P+Q$, difference $P-Q$, or product $P Q$, if well defined, generally may not have symmetry anymore. This is one of the difficulties for matrix extension with symmetry. In order for $P \pm Q$ or $P Q$ to possess some symmetry, the symmetry patterns of $P$ and $Q$ should be compatible. For example, if $S P=S Q$; that is, both $P$ and $Q$ have the same symmetry pattern, then indeed $P \pm Q$ has symmetry and $S(P \pm Q)=S P=S Q$. In the following, we discuss the compatibility of symmetry patterns of matrices of Laurent polynomials. For an $r \times s$ matrix $\mathrm{P}$ of Laurent polynomials with symmetry, we say that the symmetry of $\mathrm{P}$ is compatible or $\mathrm{P}$ has compatible symmetry, if

$$
\operatorname{SP}(z)=\left(\operatorname{S} \theta_{1}\right)^{\star}(z) \operatorname{S} \theta_{2}(z)
$$

for some $1 \times r$ vector $\theta_{1}$ and $1 \times s$ vector $\theta_{2}$ of Laurent polynomials with symmetry. For an $r \times s$ matrix $\mathrm{P}$ and an $s \times t$ matrix $\mathrm{Q}$ of Laurent polynomials with symmetry, we say that $(\mathrm{P}, \mathrm{Q})$ has mutually compatible symmetry if

$$
\mathrm{SP}(z)=\left(\mathrm{S} \theta_{1}\right)^{\star}(z) \mathrm{S} \theta(z) \text { and } \mathrm{SQ}(z)=(\mathrm{S} \theta)^{\star}(z) \mathrm{S} \theta_{2}(z)
$$

for some $1 \times r, 1 \times s, 1 \times t$ row vectors $\theta_{1}, \theta, \theta_{2}$ of Laurent polynomials with symmetry, respectively. If $(P, Q)$ has mutually compatible symmetry as in (4.4), then it is easy to verify that their product $P Q$ has compatible symmetry and in fact $S(P Q)=$ $\left(\mathrm{S} \theta_{1}\right)^{\star} \mathrm{S} \theta_{2}$. An $s \times s$ matrix $\mathrm{V}$ of Laurent polynomials is strongly invertible if $\mathrm{V}^{-1}$ is also a matrix of Laurent polynomials, or equivalently, the determinant of $\mathrm{V}$ is a nonzero monomial.

Our main result in this section is as follows:

Theorem 4.1. Let $\mathrm{P}$ be an $r \times s$ matrix, with $1 \leqslant r \leqslant s$, of Laurent polynomials taking the form

$$
\mathrm{P}(z)=\mathrm{Q}_{0}(z) D_{0}, \quad z \in \mathbb{C} \backslash\{0\},
$$

where $\mathrm{Q}_{0}$ is an $r \times s$ matrix of Laurent polynomials in $\mathbb{F}\left[z, z^{-1}\right]$ and $D_{0}$ is an $s \times s$ diagonal matrix with all the entries of $D_{0} D_{0}^{\star}$ in $\mathbb{F}$. Then: 
(1) the symmetry pattern of $\mathrm{P}$ is compatible: $\mathrm{SP}=\left(\mathrm{S} \theta_{1}\right)^{\star} \mathrm{S} \theta_{2}$ for some $1 \times r$ vector $\theta_{1}$ and some $1 \times s$ vector $\theta_{2}$ of Laurent polynomials with symmetry;

(2) $\mathrm{P}$ is paraunitary: $\mathrm{P}(z) \mathrm{P}^{\star}(z)=I_{r}$ for all $z \in \mathbb{C} \backslash\{0\}$,

if and only if, there exists an $s \times s$ square extension matrix $\mathrm{P}_{e}$ of Laurent polynomials such that

(i) $\left[I_{r}, 0\right] \mathrm{P}_{e}=\mathrm{P}$; that is, the submatrix of the first $r$ rows of $\mathrm{P}_{e}$ is $\mathrm{P}$;

(ii) $\mathrm{P}_{e}$ is paraunitary: $\mathrm{P}_{e}(z) \mathrm{P}_{e}^{\star}(z)=I_{s}$ for all $z \in \mathbb{C} \backslash\{0\}$;

(iii) the symmetry pattern of $\mathrm{P}_{e}$ is compatible: $\mathrm{SP}_{e}=(\mathrm{S} \theta)^{\star} \mathrm{S} \theta_{2}$ for some $1 \times s$ vector $\theta$ of Laurent polynomials with symmetry;

(iv) $\mathrm{P}_{e}$ can be represented as a product of some $s \times s$ elementary matrices:

$$
\mathrm{P}_{e}(z)=D_{e} \mathrm{P}_{J+1}(z) \mathrm{P}_{J}(z) \cdots \mathrm{P}_{1}(z) \mathrm{P}_{0}(z) D_{0}, \quad z \in \mathbb{C} \backslash\{0\},
$$

where

(a) $D_{e}$ is an $s \times s$ diagonal matrix with all the entries of $D_{e} D_{e}^{\star}$ in $\mathbb{F}$;

(b) $\mathrm{P}_{j}, 1 \leqslant j \leqslant J$ are elementary: $\mathrm{P}_{j}$ is strongly invertible, has coefficients in $\mathbb{F}$, and $\operatorname{fupp}\left(\mathrm{P}_{j}\right) \subseteq[-1,1]$;

(c) $\left(\mathrm{P}_{j+1}, \mathrm{P}_{j}\right)$ is mutually compatible for all $0 \leqslant j \leqslant J$;

(d) $\mathrm{P}_{0}=\mathrm{U}_{\mathrm{S}_{\theta_{2}}}^{\star}$ and $\mathrm{P}_{J+1}=\operatorname{diag}\left(\mathrm{U}_{\mathrm{S}_{1}}, I_{s-r}\right)$, where $\mathrm{U}_{\mathrm{S}_{1}}$ and $\mathrm{U}_{\mathrm{S}_{\theta_{2}}}$ are products of a permutation matrix with a diagonal matrix of monomials, as defined in (4.7);

(e) the length of $\mathrm{P}_{e}$ is controlled by that of $\mathrm{P}$ in the following sense:

$$
\operatorname{len}\left(\left[\mathrm{P}_{e}\right]_{j, k}\right) \leqslant \max _{1 \leqslant n \leqslant r} \operatorname{len}\left([\mathrm{P}]_{n, k}\right) \quad \text { for } \quad 1 \leqslant j, k \leqslant s .
$$

We make some remarks about Theorem 4.1. The integer $J$ in item (iv) of Theorem 4.1 always satisfies $J \leqslant\lceil\operatorname{len}(\mathrm{P}) / 2\rceil$. Here $\lceil\cdot\rceil$ denotes the ceiling function such that $\lceil x\rceil=n$ if $n-1<x \leqslant n$ for some integer $n$. Each of the elementary matrices $\mathrm{P}_{j}, j=1, \ldots, J$ is a product of a constant diagonal matrix in $\mathbb{F}$ and a matrix of Laurent polynomials in $\mathbb{F}\left[z, z^{-1}\right]$ with its length no greater than 2 . The matrices $\mathrm{P}_{0}$ and $\mathrm{P}_{J+1}$ are two matrices of Laurent polynomials in $\mathbb{F}\left[z, z^{-1}\right]$ reducing the symmetry pattern of $\mathrm{P}$ into a standard simplified form. For more detail, see subsections below and Algorithm 2 .

Theorem 4.1 states that an $r \times s$ paraunitary matrix $\mathrm{P}$ having compatible symmetry can be extended into a square paraunitary matrix $\mathrm{P}_{e}$ having a special cascade structure as in (4.6) and having compatible symmetry structure. In the rest of this section, we shall prove Theorem 4.1 and provide an algorithm for it (for its illustration, see Examples 6.1-6.4 in Section 6). We follow the main idea in the proof of [10. Theorem 1]. The key difference is that we need to take into account the special form of $\mathrm{P}=\mathrm{Q} D_{0}$ and construct paraunitary matrices having certain form as well. The proof for the sufficiency part of Theorem 4.1 is straightforward. The proof for the necessity part is constructive with three major steps -initialization, support reduction, and finalization - for deriving a desired matrix $\mathrm{P}_{e}$ in Theorem 4.1 from $P$. The step of initialization normalizes the symmetry pattern of $P$ to a standard form. The step of support reduction is the main body of the proof, producing a sequence of elementary matrices $A_{1}, \ldots, A_{J}$ that reduce the length of $\mathrm{P}$ to 0 . The step of finalization generates the desired matrix $\mathrm{P}_{e}$ as in Theorem 4.1. The following subsections provide details for the three major steps and the corresponding algorithm. 
4.1. Initialization. Let $\theta$ be a $1 \times s$ row vector of Laurent polynomials with symmetry such that $\mathrm{S} \theta=\left[\varepsilon_{1} z^{c_{1}}, \ldots, \varepsilon_{s} z^{c_{s}}\right]$ for some $\varepsilon_{1}, \ldots, \varepsilon_{s} \in\{-1,1\}$ and $c_{1}, \ldots, c_{s} \in$ $\mathbb{Z}$. Then the symmetry of every entry in the vector $\theta \operatorname{diag}\left(z^{-\left\lceil c_{1} / 2\right\rceil}, \ldots, z^{-\left\lceil c_{s} / 2\right\rceil}\right)$ belongs to $\left\{ \pm 1, \pm z^{-1}\right\}$. Moreover, there is a permutation matrix $E_{\theta}$ to regroup these four types of symmetries together so that

$$
\mathrm{S}\left(\theta \mathrm{U}_{\mathrm{S} \theta}\right)=\left[\mathbf{1}_{s_{1}},-\mathbf{1}_{s_{2}}, z^{-1} \mathbf{1}_{s_{3}},-z^{-1} \mathbf{1}_{s_{4}}\right],
$$

where $\mathrm{U}_{\mathrm{S} \theta}:=\operatorname{diag}\left(z^{-\left\lceil c_{1} / 2\right\rceil}, \ldots, z^{-\left\lceil c_{s} / 2\right\rceil}\right) E_{\theta}$ and $s_{1}, \ldots, s_{4}$ are nonnegative integers uniquely determined by $\mathrm{S} \theta$ such that $s_{1}+\cdots+s_{4}=s$.

Since $\mathrm{P}$ satisfies $\mathrm{SP}=\left(\mathrm{S} \theta_{1}\right)^{\star} \mathrm{S} \theta_{2}$, the matrix $\tilde{\mathrm{P}}:=\mathrm{U}_{\mathrm{S} \theta_{1}}^{\star} \mathrm{PU} \mathrm{S}_{\theta_{2}}$ must have the symmetry pattern

$$
\mathrm{SP}=\left[\mathbf{1}_{r_{1}},-\mathbf{1}_{r_{2}}, z \mathbf{1}_{r_{3}},-z \mathbf{1}_{r_{4}}\right]^{\top}\left[\mathbf{1}_{s_{1}},-\mathbf{1}_{s_{2}}, z^{-1} \mathbf{1}_{s_{3}},-z^{-1} \mathbf{1}_{s_{4}}\right] .
$$

Note that $U_{\mathrm{S}_{1}}$ and $U_{\mathrm{S}_{2}}$ do not increase the length of $\mathrm{P}$. Moreover,

$$
\tilde{\mathrm{P}}=\mathrm{U}_{\mathrm{S} \theta_{1}}^{\star} \mathrm{PU}_{\mathrm{S}_{\theta_{2}}}=\mathrm{U}_{\mathrm{S} \theta_{1}}^{\star} \mathrm{Q}_{0} D_{0} \mathrm{U}_{\mathrm{S}_{2}}=\mathrm{U}_{\mathrm{S} \theta_{1}}^{\star} \mathrm{Q}_{0} \mathrm{U}_{\mathrm{S} \theta_{2}}\left(\mathrm{U}_{\mathrm{S} \theta_{2}}^{\star} D_{0} \mathrm{U}_{\mathrm{S} \theta_{2}}\right)=: \tilde{\mathrm{Q}}_{0},
$$

where $\tilde{D}_{0}:=\mathrm{U}_{\mathrm{S} \theta_{2}}^{\star} D_{0} \mathrm{U}_{\mathrm{S} \theta_{2}}$ is a diagonal constant matrix with all the entries of $\tilde{D}_{0} \tilde{D}_{0}^{\star}$ in $\mathbb{F}$.

4.2. Support reduction. $\tilde{P}$ takes the following form:

$$
\begin{aligned}
\tilde{\mathrm{P}} & =\left[\begin{array}{cccc}
F_{11} & -F_{21} & G_{31} & -G_{41} \\
-F_{12} & F_{22} & -G_{32} & G_{42} \\
\hline \mathbf{0} & \mathbf{0} & F_{31} & -F_{41} \\
\mathbf{0} & \mathbf{0} & -F_{32} & F_{42}
\end{array}\right] z^{-k}+\left[\begin{array}{cccc}
F_{51} & -F_{61} & G_{71} & -G_{81} \\
-F_{52} & F_{61} & -G_{72} & G_{82} \\
\hline G_{11} & -G_{21} & F_{71} & -F_{81} \\
-G_{12} & G_{22} & -F_{72} & F_{82}
\end{array}\right] z^{-k+1} \\
& +\sum_{n=2-k}^{k-2} \operatorname{coeff}(\tilde{\mathrm{P}}, n) z^{n}+\left[\begin{array}{ccccc}
F_{51} & F_{61} & G_{31} & G_{41} \\
F_{52} & F_{61} & G_{32} & G_{42} \\
\hline G_{51} & G_{61} & F_{71} & F_{81} \\
G_{52} & G_{62} & F_{72} & F_{82}
\end{array}\right] z^{k-1}+\left[\begin{array}{cccc}
F_{11} & F_{21} & \mathbf{0} & \mathbf{0} \\
F_{12} & F_{22} & \mathbf{0} & \mathbf{0} \\
\hline G_{11} & G_{21} & F_{31} & F_{41} \\
G_{12} & G_{22} & F_{32} & F_{42}
\end{array}\right] z^{k}
\end{aligned}
$$

with at least one of $\operatorname{coeff}(\tilde{\mathrm{P}}, k)$ and $\operatorname{coeff}(\tilde{\mathrm{P}},-k)$ being nonzero, where $k \geqslant 1$ is an integer, all $F_{j \ell}$ 's and $G_{j \ell}$ 's are constant matrices in $\mathbb{F}$, and $F_{11}, F_{22}, F_{31}, F_{42}$ are constant matrices of size $r_{1} \times s_{1}, r_{2} \times s_{2}, r_{3} \times s_{3}, r_{4} \times s_{4}$, respectively.

We next construct an $s \times s$ paraunitary matrix $\mathrm{A}_{\tilde{p}}$ having the following properties:

(1) $\mathrm{A}_{\tilde{\mathrm{P}}}=\tilde{D}_{0}^{\star} \mathrm{V}_{\tilde{\mathrm{P}}} \tilde{D}_{1}$ for some strongly invertible matrix $\mathrm{\vee}_{\tilde{\mathrm{P}}}$ of Laurent polynomials in $\mathbb{F}\left[z, z^{-1}\right]$ and some diagonal matrix $\tilde{D}_{1}$ with all the entries of $\tilde{D}_{1} \tilde{D}_{1}^{\star}$ in $\mathbb{F}$;

(2) $\operatorname{fupp}\left(\mathrm{A}_{\tilde{\mathrm{P}}}\right) \subseteq[-1,1]$ and $\operatorname{len}\left(\tilde{\mathrm{P}} \mathrm{A}_{\tilde{\mathrm{P}}}\right)=\operatorname{len}(\tilde{\mathrm{P}})-\operatorname{len}\left(\mathrm{A}_{\tilde{\mathrm{P}}}\right)$; that is, $\mathrm{A}_{\tilde{\mathrm{P}}}$ is elementary and reduces the length of $P$ by that of $A_{\tilde{P}}$;

(3) $A_{\tilde{P}}$ has compatible symmetry and $\tilde{P} A_{\tilde{P}}$ satisfies

$$
\mathrm{S}\left(\tilde{\mathrm{P}} \mathrm{A}_{\tilde{\mathrm{P}}}\right)=\left[\mathbf{1}_{r_{1}},-\mathbf{1}_{r_{2}}, z \mathbf{1}_{r_{3}},-z \mathbf{1}_{r_{4}}\right]^{\top}\left[\mathbf{1}_{s_{1}^{\prime}},-\mathbf{1}_{s_{2}^{\prime}}, z^{-1} \mathbf{1}_{s_{3}^{\prime}},-z^{-1} \mathbf{1}_{s_{4}^{\prime}}\right],
$$

for some nonnegative integers $s_{1}^{\prime}, \ldots, s_{4}^{\prime}$ such that $s_{1}^{\prime}+\cdots+s_{4}^{\prime}=s$;

(4) up to a permutation, $\left[\mathrm{A}_{\tilde{\mathrm{P}}}\right]_{j,:}=\left(\left[\mathrm{A}_{\tilde{\mathrm{P}}}\right]_{:, j}\right)^{\top}=\mathbf{e}_{j}$, provided $[\tilde{\mathrm{P}}]_{:, j}=\mathbf{0}$.

Recall that "up to a permutation" in the above item (4) means that there exists a permutation matrix $E$ such that $\left[\mathrm{A}_{\tilde{\mathrm{P}}} E\right]_{j,:}=\left[\mathrm{A}_{\tilde{\mathrm{P}}} E\right]_{:, j}=\mathbf{e}_{j}$ provided $[\tilde{\mathrm{P}}]_{:, j}=\mathbf{0}$. Such a paraunitary matrix $A_{\tilde{P}}$ is of the form $A_{\tilde{P}}=B_{[-k, k]} B_{\tilde{P}_{1}}$. The construction of $\mathrm{B}_{[-k, k]}$ consists of two parts: $\left\{\mathrm{B}_{1}, \ldots, \mathrm{B}_{r}\right\}$ and $\mathrm{B}_{(-k, k)}$. The first part $\left\{\mathrm{B}_{1}, \ldots, \mathrm{B}_{r}\right\}$ 
is constructed recursively for each of the $r$ rows of $\tilde{\mathrm{P}}$ so that $\tilde{\mathrm{P}}_{0}:=\tilde{\mathrm{P}} \mathrm{B}_{1} \cdots \mathrm{B}_{r}$ reduces $\tilde{\mathrm{P}}$ to a special form as follows:

$$
\left[\begin{array}{cccc}
\mathbf{0} & \mathbf{0} & \tilde{G}_{31} & -\tilde{G}_{41} \\
\mathbf{0} & \mathbf{0} & -\tilde{G}_{32} & \tilde{G}_{42} \\
\hline \mathbf{0} & \mathbf{0} & \mathbf{0} & \mathbf{0} \\
\mathbf{0} & \mathbf{0} & \mathbf{0} & \mathbf{0}
\end{array}\right] z^{-k}+\cdots+\left[\begin{array}{cccc}
\mathbf{0} & \mathbf{0} & \mathbf{0} & \mathbf{0} \\
\mathbf{0} & \mathbf{0} & \mathbf{0} & \mathbf{0} \\
\hline \tilde{G}_{11} & \tilde{G}_{21} & \mathbf{0} & \mathbf{0} \\
\tilde{G}_{12} & \tilde{G}_{22} & \mathbf{0} & \mathbf{0}
\end{array}\right] z^{k} .
$$

If both coeff $\left(\tilde{\mathrm{P}}_{0},-k\right) \neq \mathbf{0}$ and $\operatorname{coeff}\left(\tilde{\mathrm{P}}_{0}, k\right) \neq \mathbf{0}$, then the second part $\mathrm{B}_{(-k, k)}$ is further constructed so that $\tilde{\mathrm{P}}_{1}:=\tilde{\mathrm{P}}_{0} \mathrm{~B}_{(-k, k)}$ satisfies either $\operatorname{fupp}\left(\tilde{\mathrm{P}}_{1}\right) \subseteq[-k+1, k]$ or $\operatorname{fsupp}\left(\tilde{\mathrm{P}}_{1}\right) \subseteq[-k, k-1]$. $\mathrm{B}_{\tilde{\mathrm{P}}_{1}}$ is then constructed so that $\operatorname{fsupp}\left(\tilde{\mathrm{P}}_{1} \mathrm{~B}_{\tilde{\mathrm{P}}_{1}}\right) \subseteq[-k+$ $1, k-1]$.

4.2.1. Construction of $\mathrm{B}_{[-k, k]}$. The following lemma is needed for the construction of $\mathrm{B}_{1}, \ldots, \mathrm{B}_{r}$, and we postpone its proof to Section 7

Lemma 4.2. Let $\mathrm{p}$ be $a 1 \times s$ row vector of Laurent polynomials with symmetry satisfying $\mathrm{p}(z) \mathrm{p}^{\star}(z)=1$ for all $z \in \mathbb{C} \backslash\{0\}$ and $\mathrm{p}(z)=\mathrm{q}_{0}(z) D_{0}, z \in \mathbb{C} \backslash\{0\}$ for some $1 \times s$ vector $\mathrm{q}_{0}$ of Laurent polynomials in $\mathbb{F}\left[z, z^{-1}\right]$ and some $s \times s$ diagonal matrix $D_{0}$ with all the entries of $D_{0} D_{0}^{\star}$ in $\mathbb{F}$. Suppose $\operatorname{fsupp}(\mathrm{p})=\left[k_{1}, k_{2}\right]$ with $k_{2}-k_{1} \geqslant 2$. Then there exists an $s \times s$ paraunitary matrix $\mathrm{B}_{\mathrm{p}}$ satisfying all the following properties:

(i) $\mathrm{B}_{\mathrm{p}}=D_{0}^{\star} \mathrm{V} D_{1}$ for some strongly invertible matrix $\mathrm{V}$ of Laurent polynomials in $\mathbb{F}\left[z, z^{-1}\right]$ and some diagonal matrix $D_{1}$ with all the entries of $D_{1} D_{1}^{\star}$ in $\mathbb{F}$;

(ii) $\operatorname{fsupp}\left(\mathrm{B}_{\mathrm{p}}\right)=[-1,1]$ and $\mathrm{SB}_{\mathrm{p}}=(\mathrm{Sp})^{\star} \mathrm{Sp}$; that is, $\mathrm{B}_{\mathrm{p}}$ has compatible symmetry with its coefficients supported inside $[-1,1]$;

(iii) $\operatorname{fsupp}\left(\mathrm{pB}_{\mathrm{p}}\right)=\left[k_{1}+1, k_{2}-1\right]$ and $\mathrm{S}\left(\mathrm{pB}_{\mathrm{p}}\right)=\mathrm{S}(\mathrm{p})$; that is, $\mathrm{B}_{\mathrm{p}}$ reduces the length of $\mathrm{p}$ exactly by 2 and preserves the symmetry pattern of $\mathrm{p}$;

(iv) for any vector $\tilde{\mathrm{p}}$ of Laurent polynomials such that $\tilde{\mathrm{p}}(z) \mathrm{p}^{\star}(z)=0$ for all $z \in$ $\mathbb{C} \backslash\{0\}$ and $\mathrm{S} \tilde{\mathrm{p}}(z)=\epsilon z^{k_{0}} \mathrm{Sp}(z)$ for some $\epsilon \in\{-1,1\}$ and some $k_{0} \in \mathbb{Z}$, then $\mathrm{S}\left(\tilde{\mathrm{p}} \mathrm{B}_{\mathrm{p}}\right)=\mathrm{S} \tilde{\mathrm{p}}$ and $\operatorname{fsupp}\left(\tilde{\mathrm{p}} \mathrm{B}_{\mathrm{p}}\right) \subseteq \operatorname{fsupp}(\tilde{\mathrm{p}})$; that is, $\mathrm{B}_{\mathrm{p}}$ keeps the symmetry pattern of $\tilde{\mathrm{p}}$ and does not increase the length of $\tilde{\mathrm{p}}$;

(v) up to a permutation, $\left[\mathrm{B}_{\mathrm{p}}\right]_{j,:}=\left(\left[\mathrm{B}_{\mathrm{p}}\right]_{:, j}\right)^{\top}=\mathbf{e}_{j}$, provided $[\mathrm{p}]_{j}=0$.

Suppose that $\tilde{\mathrm{P}}$ takes the form (4.9) with $k \geqslant 1$. If $\operatorname{fsupp}(\tilde{\mathrm{P}})=[-k, k-1]$ or $\operatorname{fupp}(\tilde{\mathrm{P}})=[-k+1, k]$, we simply let $\mathrm{B}_{[-k, k]}:=I_{s}, \tilde{\mathrm{P}}_{1}:=\tilde{\mathrm{P}}$, and continue to construct $\mathrm{B}_{\tilde{\mathrm{P}}_{1}}$ as in Section 4.2.2 otherwise, let us construct $\mathrm{B}_{1}, \ldots, \mathrm{B}_{r}$ and $\mathrm{B}_{(-k, k)}$ as follows. Let $\mathrm{p}_{j}:=[\tilde{\mathrm{P}}]_{j, \text { : }}$ be the $j$ th row of $\tilde{\mathrm{P}}$ and $\mathrm{B}_{0}:=I_{s}$. Suppose we have constructed $\mathrm{B}_{j-1}$ for $j \geqslant 1$. We first define $\mathrm{q}_{j}:=\mathrm{p}_{j} \mathrm{~B}_{0} \cdots \mathrm{B}_{j-1}$. Then $\mathrm{B}_{j}:=\mathrm{B}_{\mathbf{q}_{j}}$ if $\operatorname{fsupp}\left(\mathrm{p}_{j}\right)=\operatorname{fsupp}\left(\mathrm{q}_{j}\right)=[-k, k]$; otherwise $\mathrm{B}_{j}:=I_{s}$, where $\mathrm{B}_{\mathbf{q}_{j}}$ is constructed as in Lemma 4.2, Let $j \leftarrow j+1$ and repeat this process until $j=r$. Note that each $\mathrm{B}_{j}$ is of the form $\mathrm{B}_{j}=D_{j-1}^{\star} \mathrm{\vee}_{j} D_{j}$ for $j=1, \ldots, r$. Hence, $\tilde{\mathrm{P}}_{0}:=\tilde{\mathrm{PB}}_{1} \cdots \mathrm{B}_{r}$ preserves the form and symmetry pattern of $\tilde{\mathrm{P}}$ as $\tilde{\mathrm{P}}=\tilde{\mathrm{Q}}_{0} \tilde{D}_{0}$. Moreover, by [10, Lemma 2], the support of $\mathrm{B}_{1} \cdots \mathrm{B}_{r}$ is contained inside $[-1,1]$.

Thanks to the properties of $\mathrm{B}_{j}$ as in Lemma 4.2. $\tilde{\mathrm{P}}_{0}$ must take the form as in (4.10). If both coeff $\left(\tilde{\mathrm{P}}_{0},-k\right) \neq \mathbf{0}$ and coeff $\left(\tilde{\mathrm{P}}_{0}, k\right) \neq \mathbf{0}$, then the second part $\mathrm{B}_{(-k, k)}$ is further constructed so that $\tilde{\mathrm{P}}_{1}:=\tilde{\mathrm{P}}_{0} \mathrm{~B}_{(-k, k)}$ satisfies either $\operatorname{fsupp}\left(\tilde{\mathrm{P}}_{1}\right) \subseteq[-k+1, k]$ 
or $\operatorname{fsupp}\left(\tilde{\mathrm{P}}_{1}\right) \subseteq[-k, k-1]$. The following lemma is needed for us to construct $\mathrm{B}_{(-k, k)}$. We postpone its proof to Section 7 as well.

Lemma 4.3. Let $\left(\mathrm{q}_{1}, \mathrm{q}_{2}\right)$ be a pair of $1 \times s$ vectors of Laurent polynomials with symmetry satisfying $\mathrm{q}_{j}(z) \mathrm{q}_{\ell}^{\star}(z)=\delta(j-\ell)$ for all $z \in \mathbb{C} \backslash\{0\}$ and for $j, \ell=1,2$. Suppose $\mathrm{q}_{1}=\tilde{\mathrm{q}}_{1} D_{0}$ and $\mathrm{q}_{2}=\tilde{\mathrm{q}}_{2} D_{0}$ for some $1 \times s$ vectors $\tilde{\mathrm{q}}_{1}, \tilde{\mathrm{q}}_{2}$ of Laurent polynomials in $\mathbb{F}\left[z, z^{-1}\right]$ and some diagonal matrix $D_{0}$ with all the entries of $D_{0} D_{0}^{\star}$ in $\mathbb{F}$. Moreover, $\operatorname{fsupp}\left(\mathrm{q}_{1}\right)=[-k, k-1]$ and $\operatorname{fsupp}\left(\mathrm{q}_{2}\right)=[-k+1, k]$ with $k \geqslant 1$, and $\mathrm{Sq}_{2}(z)=\varepsilon z \mathrm{Sq}_{1}(z)=\varepsilon z\left[\mathbf{1}_{s_{1}},-\mathbf{1}_{s_{2}}, z^{-1} \mathbf{1}_{s_{3}},-z^{-1} \mathbf{1}_{s_{4}}\right]$ for some $\varepsilon \in\{-1,1\}$ and some nonnegative integers $s_{1}, \ldots, s_{4}$ such that $s_{1}+\cdots+s_{4}=s$. Then there exists an $s \times s$ paraunitary matrix $\mathrm{B}_{\left(\mathrm{q}_{1}, \mathrm{q}_{2}\right)}$ satisfying all the following properties:

(i) $\mathrm{B}_{\left(\mathrm{q}_{1}, \mathrm{q}_{2}\right)}=D_{0}^{\star} \mathrm{V}_{\left(\mathrm{q}_{1}, \mathrm{q}_{2}\right)} D_{1}$ for some strongly invertible matrix $\mathrm{V}_{\left(\mathrm{q}_{1}, \mathrm{q}_{2}\right)}$ of Laurent polynomials in $\mathbb{F}\left[z, z^{-1}\right]$ and some diagonal matrix $D_{1}$ with all the entries of $D_{1} D_{1}^{\star}$ in $\mathbb{F}$;

(ii) $\mathrm{SB}_{\left(\mathbf{q}_{1}, \mathbf{q}_{2}\right)}=\left[\mathbf{1}_{s_{1}},-\mathbf{1}_{s_{2}}, z \mathbf{1}_{s_{3}},-z \mathbf{1}_{s_{4}}\right]^{\top}\left[\mathbf{1}_{s_{1}},-\mathbf{1}_{s_{2}}, z^{-1} \mathbf{1}_{s_{3}},-z^{-1} \mathbf{1}_{s_{4}}\right]$ and support $\operatorname{fsupp}\left(\mathrm{B}_{\left(\mathbf{q}_{1}, \mathrm{q}_{2}\right)}\right)=[-1,1]$; that is, $\mathrm{B}_{\left(\mathbf{q}_{1}, \mathrm{q}_{2}\right)}$ has compatible symmetry with coefficients supported inside $[-1,1]$;

(iii) $\operatorname{fsupp}\left(\mathrm{q}_{1} \mathrm{~B}_{\left(\mathrm{q}_{1}, \mathrm{q}_{2}\right)}\right) \subseteq[-k+1, k-1]$ and $\operatorname{fsupp}\left(\mathrm{q}_{2} \mathrm{~B}_{\left(\mathrm{q}_{1}, \mathrm{q}_{2}\right)}\right) \subseteq[-k+1, k-1]$; that is, $\mathrm{B}_{\left(\mathbf{q}_{1}, \mathbf{q}_{2}\right)}$ reduces the length of $\left[\mathrm{q}_{1}^{\top}, \mathrm{q}_{2}^{\top}\right]^{\top}$ by 2 . Moreover, $\mathrm{S}\left(\mathrm{q}_{1} \mathrm{~B}_{\left(\mathrm{q}_{1}, \mathbf{q}_{2}\right)}\right)=$ $\mathrm{Sq}_{1}$ and $\mathrm{S}\left(\mathrm{q}_{2} \mathrm{~B}_{\left(\mathrm{q}_{1}, \mathrm{q}_{2}\right)}\right)=\mathrm{Sq}_{2}$;

(iv) if both $\left(\mathrm{p}, \mathrm{q}_{1}^{\star}\right)$ and $\left(\mathrm{p}, \mathrm{q}_{2}^{\star}\right)$ have mutually compatible symmetry and $\mathrm{pq}_{1}^{\star}=$ $\mathrm{pq}_{2}^{\star}=0$, then $\mathrm{S}\left(\mathrm{pB}_{\left(\mathrm{q}_{1}, \mathrm{q}_{2}\right)}\right)=\mathrm{Sp}$ and $\operatorname{fsupp}\left(\mathrm{pB}_{\left(\mathrm{q}_{1}, \mathrm{q}_{2}\right)}\right) \subseteq \operatorname{fsupp}(\mathrm{p})$; that is, $\mathrm{B}_{\left(\mathrm{q}_{1}, \mathrm{q}_{2}\right)}$ keeps the symmetry pattern of $\mathrm{p}$ and does not increase the length of $\mathrm{p}$;

(v) up to a permutation, $\left[\mathrm{B}_{\left(\mathrm{q}_{1}, \mathrm{q}_{2}\right)}\right]_{j,:}=\left(\left[\mathrm{B}_{\left(\mathrm{q}_{1}, \mathrm{q}_{2}\right)}\right]_{:, j}\right)^{\top}=\mathbf{e}_{j}$, provided $\left[\mathrm{q}_{1}\right]_{j}=$ $\left[\mathbf{q}_{2}\right]_{j}=0$.

Now, suppose $\tilde{\mathrm{P}}_{0}$ takes the form in (4.10). Let $\mathrm{B}_{(-k, k)}:=I_{s}$ and $\tilde{\mathrm{P}}_{1}:=\tilde{\mathrm{P}}_{0}$. Pick any two rows $\mathrm{q}_{1}, \mathrm{q}_{2}$ of $\tilde{\mathrm{P}}_{1}$ such that $\operatorname{fsupp}\left(\mathrm{q}_{1}\right)=[-k, k-1]$ and $\operatorname{fsupp}\left(\mathrm{q}_{2}\right)=[-k+$ $1, k]$. Then, the pair $\left(q_{1}, q_{2}\right)$ satisfies all the conditions in Lemma 4.3. Hence, we can construct a paraunitary matrix $\mathrm{B}_{\left(\mathbf{q}_{1}, \mathbf{q}_{2}\right)}$ having the properties as in Lemma 4.3 . Replace $\mathrm{B}_{(-k, k)}$ and $\tilde{\mathrm{P}}_{1}$ by $\mathrm{B}_{(-k, k)} \mathrm{B}_{\left(\mathbf{q}_{1}, \mathbf{q}_{2}\right)}$ and $\tilde{\mathrm{P}}_{1} \mathrm{~B}_{\left(\mathbf{q}_{1}, \mathrm{q}_{2}\right)}$, respectively. Pick another two rows $\left(\mathrm{q}_{1}, \mathrm{q}_{2}\right)$ such that $\operatorname{fsupp}\left(\mathrm{q}_{1}\right)=[-k, k-1]$ and $\operatorname{fsupp}\left(\mathrm{q}_{2}\right)=[-k+1, k]$ from the new matrix $\tilde{\mathrm{P}}_{1}$ and repeat the above process. Lemma 4.3 guarantees that this process stops in finite steps and there will be no pair $\left(q_{1}, q_{2}\right)$ of rows in $\tilde{P}_{1}$ satisfying $\operatorname{fsupp}\left(\mathrm{q}_{1}\right)=[-k, k-1]$ and $\operatorname{fsupp}\left(\mathrm{q}_{2}\right)=[-k+1, k]$. Then $\mathrm{P}_{1}$ must take the form as in (4.10) with at least one of $\operatorname{coeff}\left(\tilde{\mathrm{P}}_{1},-k\right)$ and coeff $\left(\tilde{\mathrm{P}}_{1}, k\right)$ being $\mathbf{0}$.

4.2.2. Construction of $\mathrm{B}_{\tilde{\mathrm{P}}_{1}}$. $\mathrm{B}_{\tilde{\mathrm{P}}_{1}}$ is constructed to handle the case that $\operatorname{fsupp}\left(\tilde{\mathrm{P}}_{1}\right)=$ $[-k, k-1]$ or $\operatorname{fsupp}\left(\tilde{\mathrm{P}}_{1}\right)=[-k+1, k]$ so that $\operatorname{fsupp}\left(\tilde{\mathrm{P}}_{1} \mathrm{~B}_{\tilde{\mathrm{P}}_{1}}\right) \subseteq[-k+1, k-1]$.

If $\tilde{\mathrm{P}}_{1}:=\tilde{\mathrm{P}}_{[-k, k]}$ takes the form in (4.10) with coeff $\left(\tilde{\mathrm{P}}_{1},-k\right)=\operatorname{coeff}\left(\tilde{\mathrm{P}}_{1}, k\right)=\mathbf{0}$, then we simply let $\mathrm{B}_{\tilde{\mathrm{P}}_{1}}:=I_{s}$; otherwise, one of $\operatorname{coeff}\left(\tilde{\mathrm{P}}_{1},-k\right)$ and $\operatorname{coeff}\left(\tilde{\mathrm{P}}_{1}, k\right)$ is nonzero. For this case, $\mathrm{B}_{\tilde{\mathrm{P}}_{1}}:=\operatorname{diag}\left(U_{1} \mathrm{~W}_{1}, I_{s_{3}+s_{4}}\right) E$ with $U_{1}$ and $\mathbf{W}_{1}$ being constructed with respect to $\operatorname{coeff}\left(\tilde{\mathrm{P}}_{1}, k\right) \neq \mathbf{0}$ or $\mathrm{B}_{\tilde{\mathrm{P}}_{1}}:=\operatorname{diag}\left(I_{s_{1}+s_{2}}, U_{3} \mathrm{~W}_{3}\right) E$ with $U_{3}$ and $\mathrm{W}_{3}$ being constructed with respect to $\operatorname{coeff}\left(\tilde{\mathrm{P}}_{1},-k\right) \neq \mathbf{0}$, where $E$ is a permutation matrix. Note that $\tilde{\mathrm{P}}_{1}$ is still of the form $\tilde{\mathrm{P}}_{1}=\tilde{\mathrm{Q}}_{1} \tilde{D}_{1}$ similar to $\tilde{\mathrm{P}}$. The matrices $U_{1}, \mathrm{~W}_{1}, U_{3}, \mathrm{~W}_{3}$, and $E$ are constructed as follows. 
Let $U_{1}:=\operatorname{diag}\left(U_{\tilde{G}_{1}}, U_{\tilde{G}_{2}}\right)$ and $U_{3}:=\operatorname{diag}\left(U_{\tilde{G}_{3}}, U_{\tilde{G}_{4}}\right)$ with

$$
\tilde{G}_{1}:=\left[\begin{array}{c}
\tilde{G}_{11} \\
\tilde{G}_{12}
\end{array}\right], \tilde{G}_{2}:=\left[\begin{array}{c}
\tilde{G}_{21} \\
\tilde{G}_{22}
\end{array}\right], \tilde{G}_{3}:=\left[\begin{array}{c}
\tilde{G}_{31} \\
\tilde{G}_{32}
\end{array}\right], \tilde{G}_{4}:=\left[\begin{array}{c}
\tilde{G}_{41} \\
\tilde{G}_{42}
\end{array}\right],
$$

where $U_{\tilde{G}_{1}}, \ldots, U_{\tilde{G}_{4}}$ are unitary constant matrices constructed as in Corollary 2.3. If $G_{1} G_{1}^{\star}=G_{2} G_{2}^{\star}$, one can show that $U_{G_{1}}$ and $U_{G_{2}}$ can be constructed such that $G_{1} U_{G_{1}}=[R, \mathbf{0}]$ and $G_{2} U_{G_{2}}=[R, \mathbf{0}]$.

Let $m_{1}$ and $m_{3}$ be the ranks of $\tilde{G}_{1}$ and $\tilde{G}_{3}$, respectively $\left(m_{1}=0\right.$ if coeff $\left(\tilde{\mathrm{P}}_{1}, k\right)=$ $\mathbf{0}$, and $m_{3}=0$ if $\left.\operatorname{coeff}\left(\tilde{P}_{1},-k\right)=\mathbf{0}\right)$. Note that $\tilde{G}_{1} \tilde{G}_{1}^{\star}=\tilde{G}_{2} \tilde{G}_{2}^{\star}$ and $\tilde{G}_{3} \tilde{G}_{3}^{\star}=\tilde{G}_{4} \tilde{G}_{4}^{\star}$ in view of $\tilde{\mathrm{P}}_{1} \tilde{\mathrm{P}}_{1}^{\star}=I_{r}$. The matrices $\mathrm{W}_{1}$ and $\mathrm{W}_{3}$ are then constructed to be:

$\mathrm{W}_{1}:=\left[\begin{array}{l|l|l|l}\mathrm{U}_{1} & & \mathrm{U}_{2} & \\ \hline & I_{s_{1}-m_{1}} & & \\ \hline \mathrm{U}_{2} & & \mathrm{U}_{1} & \\ \hline & & & I_{s_{2}-m_{1}}\end{array}\right], \quad \mathrm{W}_{3}:=\left[\begin{array}{l|l|l|l}\mathrm{U}_{3} & & \mathrm{U}_{4} & \\ \hline & I_{s_{3}-m_{3}} & & \\ \hline \mathrm{U}_{4} & & \mathrm{U}_{3} & \\ \hline & & & I_{s_{4}-m_{3}}\end{array}\right]$,

where $\mathrm{U}_{1}(z)=-\mathrm{U}_{2}(-z):=\frac{1+z^{-1}}{2} I_{m_{1}}$ and $\mathrm{U}_{3}(z)=\mathrm{U}_{4}(-z):=\frac{1+z}{2} I_{m_{3}}$.

Let $\mathrm{W}_{\tilde{\mathrm{P}}_{1}}:=\operatorname{diag}\left(U_{1} \mathrm{~W}_{1}, I_{s_{3}+s_{4}}\right)$ for the case that $\operatorname{coeff}\left(\tilde{\mathrm{P}}_{1}, k\right) \neq \mathbf{0}$ or $\mathrm{W}_{\tilde{\mathrm{P}}_{1}}:=$ $\operatorname{diag}\left(I_{s_{1}+s_{2}}, U_{3} \mathbf{W}_{3}\right)$ for the case that $\operatorname{coeff}\left(\tilde{\mathrm{P}}_{1},-k\right) \neq \mathbf{0}$. Then $\mathbf{W}_{\tilde{\mathrm{P}}_{1}}$ is paraunitary. By the symmetry pattern and paraunitary property of $\tilde{P}_{1}, W_{\tilde{P}_{1}}$ reduces the coefficient support of $\tilde{\mathrm{P}}_{1}$ to $[-k+1, k-1]$, i.e., $\operatorname{fsupp}\left(\tilde{\mathrm{P}}_{1} \mathrm{~W}_{\tilde{\mathrm{P}}_{1}}\right)=[-k+1$, $k-1]$. Moreover, $\mathrm{W}_{\tilde{\mathrm{P}}_{1}}$ changes the symmetry pattern of $\tilde{\mathrm{P}}_{1}$ such that $\mathrm{S}\left(\tilde{\mathrm{P}}_{1} \mathrm{~W}_{\tilde{\mathrm{P}}_{1}}\right)=$ $\left[\mathbf{1}_{r_{1}},-\mathbf{1}_{r_{2}}, z \mathbf{1}_{r_{3}},-z \mathbf{1}_{r_{4}}\right]^{\top} \mathrm{S} \theta_{0}$ with

$\mathrm{S} \theta_{0}=\left[z^{-1} \mathbf{1}_{m_{1}}, \mathbf{1}_{s_{1}-m_{1}},-z^{-1} \mathbf{1}_{m_{1}},-\mathbf{1}_{s_{2}-m_{1}}, \mathbf{1}_{m_{3}}, z^{-1} \mathbf{1}_{s_{3}-m_{3}},-\mathbf{1}_{m_{3}},-z^{-1} \mathbf{1}_{s_{4}-m_{3}}\right]$.

$E$ is then the permutation matrix such that

$$
\mathrm{S}\left(\tilde{\mathrm{P}}_{1} \mathrm{~W}_{\tilde{\mathrm{P}}_{1}}\right) E=\left[\mathbf{1}_{r_{1}},-\mathbf{1}_{r_{2}}, z \mathbf{1}_{r_{3}},-z \mathbf{1}_{r_{4}},\right]^{\top} \mathrm{S} \tilde{\theta}_{0},
$$

with S $\tilde{\theta}_{0}=\left[\mathbf{1}_{s_{1}-m_{1}+m_{3}},,-\mathbf{1}_{s_{2}-m_{1}+m_{3}}, z^{-1} \mathbf{1}_{s_{3}-m_{3}+m_{1}},-z^{-1} \mathbf{1}_{s_{4}-m_{3}+m_{1}}\right]=\left(\mathrm{S} \theta_{0}\right) E$.

4.3. Finalization. In summary, $A_{\tilde{P}}=B_{[-k, k]} B_{\tilde{P}_{1}}$ reduces the support of $\tilde{P}$ and preserves the compatible symmetry of $\tilde{P}$. The properties (1), (3), and (4) of $A_{\tilde{P}}$ follow directly from the above construction while the support property (2) of $A_{\tilde{P}}$ follows from [10, Lemmas 1 and 2]. Replacing $\tilde{P}$ by $\tilde{P} A_{\tilde{P}}$ and repeating the above construction, we can construct paraunitary matrices $A_{1}, \ldots, A_{J}$ so that $\left(\mathrm{U}_{\mathrm{S} \theta_{1}}^{\star} \mathrm{PU}_{\mathrm{S}_{\theta_{2}}}\right) \mathrm{A}_{1} \cdots \mathrm{A}_{J}=\left[I_{r}, \mathbf{0}\right]$. Since each $\mathrm{A}_{j}$ is of the form $\mathrm{A}_{j}=\tilde{D}_{j-1}^{\star} \mathrm{\vee}_{j} \tilde{D}_{j}$ for some strongly invertible matrix $\mathrm{V}_{j}$ of Laurent polynomials in $\mathbb{F}\left[z, z^{-1}\right]$ and some diagonal matrices $\tilde{D}_{j-1}, \tilde{D}_{j}$ with all the entries of $\tilde{D}_{j-1} \tilde{D}_{j-1}^{\star}, \tilde{D}_{j} \tilde{D}_{j}^{\star}$ in $\mathbb{F}$, it is easy to show that the paraunitary extension matrix $\mathrm{P}_{e}:=\operatorname{diag}\left(\mathrm{U}_{\mathrm{S}_{1}}, I_{s-r}\right) \mathrm{A}_{J}^{\star} \cdots \mathrm{A}_{1}^{\star} \mathrm{U}_{\mathrm{S} \theta_{2}}^{\star}$ takes the form as in (4.6) with $\mathrm{P}_{j}:=\tilde{D}_{j} \tilde{D}_{j}^{\star} \mathrm{V}_{J}^{\star}$ for $j=1, \ldots, J-1, \mathrm{P}_{J}:=\mathrm{V}_{J}^{\star}$, and $D_{e}:=\operatorname{diag}\left(\mathrm{U}_{\mathrm{S} \theta_{1}}, I_{s-r}\right) \tilde{D}_{J}^{\star} \operatorname{diag}\left(\mathrm{U}_{\mathrm{S} \theta_{1}}^{\star}, I_{s-r}\right)$. All the properties of $\mathrm{P}_{e}$ follow directly from the properties of each $A_{\tilde{P}}$.

4.4. Algorithm. Based on the above three steps: initialization, support reduction, and finalization, we present an algorithm (see Algorithm 2) for the matrix extension problem with symmetry constraint. 
Algorithm 2. Matrix Extension with Symmetry Constraint

(a) Input. $\mathrm{P}=\mathrm{Q}_{0} D_{0}$ as in Theorem 4.1 with $\mathrm{SP}=\left(\mathrm{S} \theta_{1}\right)^{\star} \mathrm{S} \theta_{2}$ for some $1 \times r$ vector $\theta_{1}$ and $1 \times s$ vector $\theta_{2}$ of Laurent polynomials with symmetry.

(b) Output. A desired extension matrix $\mathrm{P}_{e}$ satisfying all the properties in Theorem 4.1

(c) Initialization. $\tilde{\mathrm{P}} \leftarrow \mathrm{U}_{\mathrm{S} \theta_{1}}^{\star} \mathrm{PU}_{\mathrm{S}_{\theta_{2}}} . \quad D_{1} \leftarrow \mathrm{U}_{\mathrm{S} \theta_{2}}^{\star} D_{0} \mathrm{U}_{\mathrm{S}_{\theta_{2}}} . \mathrm{P}_{0} \leftarrow \mathrm{U}_{\mathrm{S} \theta_{2}}^{\star} . J \leftarrow 1$. Then $\tilde{\mathrm{P}}$ has the symmetry pattern as in (4.8), where all nonnegative integers $r_{1}, \ldots, r_{4}, s_{1}, \ldots, s_{4}$ are uniquely determined by SP.

(d) Support Reduction.

1: while $(\operatorname{len}(\tilde{P})>0)$ do

$\tilde{\mathrm{P}}_{0} \leftarrow \tilde{\mathrm{P}} . \tilde{\mathrm{P}}_{1} \leftarrow \tilde{\mathrm{P}} .\left[k_{1}, k_{2}\right] \leftarrow \operatorname{fsupp}(\tilde{\mathrm{P}}) . \mathrm{A}_{J} \leftarrow I_{s} . k \leftarrow k_{2}$.

if $k_{2}=-k_{1}$ then

for $j=1$ to $r$ do

$\mathrm{q} \leftarrow\left[\tilde{\mathrm{P}}_{0}\right]_{j,:}$ and $\mathrm{p} \leftarrow[\tilde{\mathrm{P}}]_{j,:} . / /$ the $j$ th rows of $\tilde{\mathrm{P}}_{0}$ and $\tilde{\mathrm{P}}$.

$\left[\ell_{1}, \ell_{2}\right] \leftarrow \operatorname{fsupp}(\mathrm{q}) . \ell \leftarrow \ell_{2}-\ell_{1} . \mathrm{B}_{j} \leftarrow I_{s}$.

if $\operatorname{fsupp}(\mathrm{q})=\operatorname{fsupp}(\mathrm{p})$ and $\ell \geqslant 2$ and $\left(\ell_{1}=k_{1}\right.$ or $\left.\ell_{2}=k_{2}\right)$ then

$\mathrm{B}_{j} \leftarrow \mathrm{B}_{\mathrm{q}}=: D_{1}^{\star} \mathrm{V}_{\mathrm{q}} D_{2} . / / \mathrm{B}_{\mathrm{q}}$ is constructed by Lemma 4.2.

$\mathrm{A}_{J} \leftarrow \mathrm{A}_{J} \mathrm{~B}_{j} . \tilde{\mathrm{P}}_{0} \leftarrow \tilde{\mathrm{P}}_{0} \mathrm{~B}_{j} . D_{1} \leftarrow D_{2}$.

end if

end for

$\tilde{\mathrm{P}}_{0}$ must take the form in (4.10).

$\mathrm{B}_{(-k, k)} \leftarrow I_{s} . \tilde{\mathrm{P}}_{1} \leftarrow \tilde{\mathrm{P}}_{0} \cdot j_{1} \leftarrow 1 . j_{2} \leftarrow r_{3}+r_{4}+1$.

while $j_{1} \leqslant r_{1}+r_{2}$ and $j_{2} \leqslant r$ do

$\mathrm{q}_{1} \leftarrow\left[\tilde{\mathrm{P}}_{1}\right]_{j_{1},:} \cdot \mathrm{q}_{2} \leftarrow\left[\tilde{\mathrm{P}}_{1}\right]_{j_{2},:}$.

if $\operatorname{coeff}\left(\mathrm{q}_{1},-k\right)=\mathbf{0}$ then $j_{1} \leftarrow j_{1}+1$ end if

if $\operatorname{coeff}\left(\mathrm{q}_{2}, k\right)=\mathbf{0}$ then $j_{2} \leftarrow j_{2}+1$ end if

if $\operatorname{coeff}\left(\mathrm{q}_{1},-k\right) \neq \mathbf{0}$ and $\operatorname{coeff}\left(\mathrm{q}_{2}, k\right) \neq \mathbf{0}$ then

$\mathrm{B}_{\left(\mathrm{q}_{1}, \mathrm{q}_{2}\right)}=: D_{1}^{\star} \mathrm{V}_{\left(\mathrm{q}_{1}, \mathrm{q}_{2}\right)} D_{2} \cdot / /$ See Lemma 4.3 for the construction.

$\mathrm{B}_{(-k, k)} \leftarrow \mathrm{B}_{(-k, k)} \mathrm{B}_{\left(\mathrm{q}_{1}, \mathrm{q}_{2}\right)} . \tilde{\mathrm{P}}_{1} \leftarrow \tilde{\mathrm{P}}_{1} \mathrm{~B}_{\left(\mathrm{q}_{1}, \mathrm{q}_{2}\right)} . \mathrm{A}_{J} \leftarrow \mathrm{A}_{J} \mathrm{~B}_{\left(\mathrm{q}_{1}, \mathrm{q}_{2}\right)} . D_{1} \leftarrow$ $D_{2}$.

21:

22 :

23 :

24:

$j_{1} \leftarrow j_{1}+1 . j_{2} \leftarrow j_{2}+1$

end if

end while // end inner while loop

\section{end if}

$\tilde{\mathrm{P}}_{1}$ takes the form in (4.10) with either $\operatorname{coeff}\left(\tilde{\mathrm{P}}_{1},-k\right)=\mathbf{0}$ or $\operatorname{coeff}\left(\tilde{\mathrm{P}}_{1}, k\right)=\mathbf{0}$. $\mathrm{B}_{\tilde{\mathrm{P}}_{1}}=: D_{1}^{\star} \mathrm{V}_{\tilde{\mathrm{P}}} D_{2}$. //See Section 4.2 .2 for the construction.

27: $\quad \mathrm{A}_{J} \leftarrow \mathrm{A}_{J} \mathrm{~B}_{\tilde{\mathrm{P}}_{1}}$. Then $\mathrm{A}_{J}$ is of the form $\mathrm{A}_{J}=\tilde{D}_{1}^{\star} \mathrm{V}_{J} \tilde{D}_{2}$ and

$$
\mathrm{SP}=\left[\mathbf{1}_{r_{1}},-\mathbf{1}_{r_{2}}, z \mathbf{1}_{r_{3}},-z \mathbf{1}_{r_{4}}\right]^{\top}\left[\mathbf{1}_{s_{1}^{\prime}},-\mathbf{1}_{s_{2}^{\prime}}, z^{-1} \mathbf{1}_{s_{3}^{\prime}},-z^{-1} \mathbf{1}_{s_{4}^{\prime}}\right]
$$

for some nonnegative integers $s_{1}^{\prime}, \ldots, s_{4}^{\prime}$.

28: $\quad$ for $j=1$ to 4 do $s_{j} \leftarrow s_{j}^{\prime}$ end for

29: $\quad \tilde{\mathrm{P}} \leftarrow \tilde{\mathrm{P}} \mathrm{A}_{J} . \mathrm{P}_{J} \leftarrow \tilde{D}_{2} \tilde{D}_{2}^{\star} \mathrm{V}_{J}^{\star} . D_{1} \leftarrow D_{2} . J \leftarrow J+1$.

30: end while // end outer while loop

(e) Finalization. $\tilde{\mathrm{P}}=\operatorname{diag}\left(F_{1}, F_{2}, F_{3}, F_{4}\right) D_{1}=\operatorname{diag}\left(\tilde{F}_{1}, \tilde{F}_{2}, \tilde{F}_{3}, \tilde{F}_{4}\right)$ for some $r_{j} \times$ $s_{j}$ constant matrices $F_{j}$ in $\mathbb{F}, j=1, \ldots, 4$. Let $U:=\operatorname{diag}\left(U_{\tilde{F}_{1}}, U_{\tilde{F}_{2}}, U_{\tilde{F}_{3}}, U_{\tilde{F}_{4}}\right)=$ $D_{1}^{\star} V_{0} D_{2}$ so that $\tilde{\mathrm{P}} U=\left[I_{r}, \mathbf{0}\right]$. Define $\mathrm{P}_{J}:=V_{0}^{\star}, \mathrm{P}_{J+1}:=\operatorname{diag}\left(\mathrm{U}_{\mathrm{S} \theta_{1}}, I_{s-r}\right)$, and $D_{e}:=\mathrm{P}_{J+1} D_{2}^{\star} \mathrm{P}_{J+1}^{\star}$. 


\section{Application to orthogonal Wavelet filter Banks WITH SYMMETRY STRUCTURE}

In this section, we shall discuss the application of our results on the matrix extension problem with symmetry in Section 4 to the construction of $\mathrm{d}$-orthogonal wavelet filter banks with symmetry structure in electronic engineering and wavelet analysis.

Symmetry of the filters in a filter bank is a very much desired property in many applications. We say that a low-pass filter $a$ with multiplicity $r$ has symmetry if its symbol a satisfies

$$
\mathrm{a}(z)=\operatorname{diag}\left(\varepsilon_{1} z^{\mathrm{d} c_{1}}, \ldots, \varepsilon_{r} z^{\mathrm{d} c_{r}}\right) \mathrm{a}\left(z^{-1}\right) \operatorname{diag}\left(\varepsilon_{1} z^{-c_{1}}, \ldots, \varepsilon_{r} z^{-c_{r}}\right)
$$

for some $\varepsilon_{1}, \ldots, \varepsilon_{r} \in\{-1,1\}$ and some $c_{1}, \ldots, c_{r} \in \mathbb{R}$ such that

$$
\mathrm{d} c_{\ell}-c_{j} \in \mathbb{Z} \quad \forall \ell, j=1, \ldots, r .
$$

Under the symmetry condition in (5.1), to apply Theorem 4.1 we first show that there exists a suitable paraunitary matrix $U$ acting on $P_{a}:=\left[a^{[0]}, \ldots, a^{[d-1]}\right]$ so that $P:=\sqrt{d} P_{a} U$ has compatible symmetry. Note that $P_{a}$ itself may not have any symmetry. More importantly, $\mathrm{P}$ has a special structure $\mathrm{P}=\mathrm{Q}_{0} D_{0}$ and satisfies all the conditions in Theorem 4.1 .

Lemma 5.1. Let $a: \mathbb{Z} \rightarrow \mathbb{F}^{r \times r}$ be a d-orthogonal wavelet filter with multiplicity $r$ whose symbol a satisfies the symmetry property in (5.1). Let $\mathrm{P}_{\mathrm{a}}:=\left[\mathrm{a}^{[0]}, \ldots, \mathrm{a}^{[\mathrm{d}-1]}\right]$, where $\mathrm{a}^{[0]}, \ldots, \mathrm{a}^{[\mathrm{d}-1]}$ are polyphase components of $\mathrm{a}$. Then there exists $a \mathrm{~d} r \times \mathrm{d} r$ paraunitary matrix $\mathrm{U}$ such that $\mathrm{P}:=\sqrt{\mathrm{d}} \mathrm{P}_{\mathrm{a}} \mathrm{U}$ satisfies the following properties:

(i) $\mathrm{P}=\mathrm{Q}_{0} D_{0}$ for some $r \times \mathrm{d} r$ matrix $\mathrm{Q}_{0}$ of Laurent polynomials in $\mathbb{F}\left[z, z^{-1}\right]$ and some $\mathrm{d} r \times \mathrm{d} r$ diagonal matrix $D_{0}$ with all the entries of $D_{0} D_{0}^{\star}$ in $\mathbb{F}$;

(ii) $\mathrm{P}$ has compatible symmetry; that is $\mathrm{SP}=\left(\mathrm{S} \theta_{1}\right)^{\star} \mathrm{S} \theta_{2}$ for some $1 \times r$ vector $\theta_{1}$ and some $1 \times \mathrm{d} r$ vector $\theta_{2}$ of Laurent polynomials with symmetry.

Proof. From (5.1), we deduce that

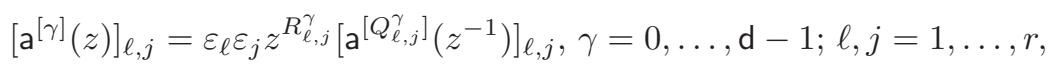

where $\gamma, Q_{\ell, j}^{\gamma} \in \Gamma:=\{0, \ldots, \mathrm{d}-1\}$ and $R_{\ell, j}^{\gamma}, Q_{\ell, j}^{\gamma}$ are uniquely determined by

$$
\mathrm{d} c_{\ell}-c_{j}-\gamma=\mathrm{d} R_{\ell, j}^{\gamma}+Q_{\ell, j}^{\gamma} \quad \text { with } \quad R_{\ell, j}^{\gamma} \in \mathbb{Z}, Q_{\ell, j}^{\gamma} \in \Gamma .
$$

Since $\mathrm{d} c_{\ell}-c_{j} \in \mathbb{Z}$ for all $\ell, j=1, \ldots, r$, we have $c_{\ell}-c_{j} \in \mathbb{Z}$ for all $\ell, j=1, \ldots, r$ and therefore, $Q_{\ell, j}^{\gamma}$ is independent of $\ell$. Consequently, by (5.3), for every $1 \leqslant j \leqslant r$, the $j$ th column of the matrix $a^{[\gamma]}$ is a flipped version of the $j$ th column of the matrix

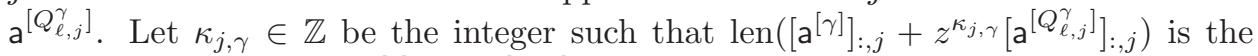
smallest. Define $\mathrm{P}_{\tilde{a}}:=\left[\tilde{a}^{[0]}, \ldots, \tilde{a}^{[d-1]}\right]$ as follows:

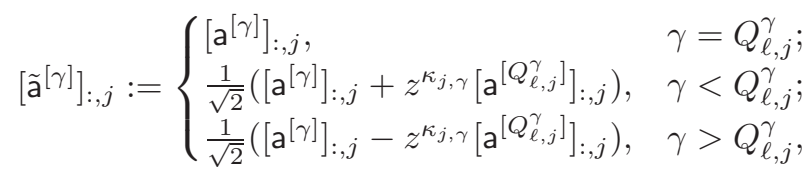

where $\left[\mathrm{a}^{[\gamma]}\right]_{:, j}$ denotes the $j$ th column of $\mathrm{a}^{[\gamma]}$. Let $\mathrm{U}$ denote the unique transform matrix corresponding to (5.5). It is easily seen that $U$ is paraunitary and can be rewritten as a product of a strongly invertible matrix of Laurent polynomials with a diagonal matrix whose diagonal entries are either 1 or $\frac{1}{\sqrt{2}}$. 
Define $\mathrm{P}:=\sqrt{\mathrm{d}} \mathrm{P}_{\tilde{\mathrm{a}}}=\sqrt{\mathrm{d}} \mathrm{P}_{\mathrm{a}} \mathrm{U}$. Then item (i) holds. We now show that $\mathrm{P}$ has compatible symmetry. Indeed, by (5.3) and (5.5),

$$
\left[\operatorname{Sãa}^{[\gamma]}(z)\right]_{\ell, j}=\operatorname{sgn}\left(Q_{\ell, j}^{\gamma}-\gamma\right) \varepsilon_{\ell} \varepsilon_{j} z^{R_{\ell, j}^{\gamma}+\kappa_{j, \gamma}},
$$

where $\operatorname{sgn}(x)=1$ for $x \geqslant 0$ and $\operatorname{sgn}(x)=-1$ for $x<0$. By (5.4) and noting that $Q_{\ell, j}^{\gamma}$ is independent of $\ell$, we have

$$
\frac{\left[\mathrm{Sãa}^{[\gamma]}(z)\right]_{\ell, j}}{\left[\mathrm{Sãa}^{[\gamma]}(z)\right]_{n, j}}=\varepsilon_{\ell} \varepsilon_{n} z^{R_{\ell, j}^{\gamma}-R_{n, j}^{\gamma}}=\varepsilon_{\ell} \varepsilon_{n} z^{c_{\ell}-c_{n}}, \quad \ell, n=1, \ldots, r,
$$

which is equivalent to saying that $\mathrm{P}$ has compatible symmetry.

In view of Lemma 5.1 and Theorem 4.1 we have the following result.

Theorem 5.2. Let $a: \mathbb{Z} \rightarrow \mathbb{F}^{r \times r}$ be a d-orthogonal wavelet filter with multiplicity $r$ whose symbol a satisfies the symmetry property in (5.1). Then there exist highpass filters $b_{1}, \ldots, b_{\mathrm{d}-1}: \mathbb{Z} \rightarrow \mathbb{C}^{r \times r}$ such that $\left\{a ; b_{1}, \ldots, b_{\mathrm{d}-1}\right\}$ forms a $\mathrm{d}$-orthogonal wavelet filter bank with $b_{m}=D_{m} \tilde{b}_{m}, m=1, \ldots, \mathrm{d}-1$, where $\tilde{b}_{m}: \mathbb{Z} \rightarrow \mathbb{F}^{r \times r}$ has coefficients in $\mathbb{F}$ and $D_{m}$ is a diagonal matrix with all the entries of $D_{m} D_{m}^{\star}$ in $\mathbb{F}$. Moreover, all filters $b_{m}, m=1, \ldots, \mathrm{d}-1$, have the following symmetry property:

$$
\mathrm{b}_{m}(z)=\operatorname{diag}\left(\varepsilon_{1}^{m} z^{\mathrm{d} c_{1}^{m}}, \ldots, \varepsilon_{r}^{m} z^{\mathrm{d} c_{r}^{m}}\right) \mathrm{b}_{m}\left(z^{-1}\right) \operatorname{diag}\left(\varepsilon_{1} z^{-c_{1}}, \ldots, \varepsilon_{r} z^{-c_{r}}\right),
$$

where $c_{\ell}^{m}:=\left(k_{\ell}^{m}-k_{\ell}\right)+c_{\ell} \in \mathbb{R}, \varepsilon_{\ell}^{m} \in\{-1,1\}$, and $k_{\ell}^{m}, k_{\ell} \in \mathbb{Z}$, for $\ell, j=1, \ldots, r$ and $m=1, \ldots, \mathrm{d}-1$.

Proof. Let $\mathrm{P}_{\mathrm{a}}:=\left[\mathrm{a}^{[0]}, \ldots, \mathrm{a}^{[\mathrm{d}-1]}\right]$. By Lemma 5.1] there exists a paraunitary matrix $\mathrm{U}$ such that $\mathrm{P}:=\sqrt{\mathrm{d}} \mathrm{P}_{\mathrm{a}} \mathrm{U}$ has compatible symmetry: $\mathrm{SP}=\left[\varepsilon_{1} z^{k_{1}}, \ldots, \varepsilon_{r} z^{k_{r}}\right]^{\top} \mathrm{S} \theta$ for some $k_{1}, \ldots, k_{r} \in \mathbb{Z}$ and some $1 \times \mathrm{d} r$ row vector $\theta$ of Laurent polynomials with symmetry. Moreover, $\mathrm{P}$ is of the form $\mathrm{P}=\mathrm{Q}_{0} D_{0}$, where $\mathrm{Q}_{0}$ is a matrix of Laurent polynomials in $\mathbb{F}\left[z, z^{-1}\right]$ and $D_{0}$ is a diagonal matrix with all the entries of $D_{0} D_{0}^{\star}$ in $\mathbb{F}$. Because a is a $\mathrm{d}$-orthogonal wavelet filter, we have $\mathrm{P}_{\mathrm{a}} \mathrm{P}_{\mathrm{a}}^{\star}=\mathrm{d}^{-1} I_{r}$ and consequently $\mathrm{P}$ is a paraunitary matrix satisfying $\mathrm{PP}^{\star}=I_{r}$. Now by Theorem 4.1 , there exists a paraunitary extension matrix $\mathrm{P}_{e}$ of the form $\mathrm{P}_{e}=D_{e} \mathrm{Q}_{e} D_{0}$ having the following compatible symmetry pattern:

$$
\left[\varepsilon_{1} z^{k_{1}}, \ldots, \varepsilon_{r} z^{k_{r}}, \varepsilon_{1}^{1} z^{k_{1}^{1}}, \ldots, \varepsilon_{r}^{1} z^{k_{r}^{1}}, \ldots, z^{k_{1}^{\mathrm{d}-1}}, \ldots, \varepsilon_{r}^{\mathrm{d}-1} z^{k_{r}^{\mathrm{d}-1}}\right]^{\top} \mathrm{S} \theta(z):=\mathrm{SP}_{e}(z) .
$$

Rewrite $\mathrm{P}_{e}=\left(\tilde{\mathbf{a}}_{m}^{[\gamma]}\right)_{0 \leqslant m, \gamma \leqslant \mathrm{d}-1}$ as a $\mathrm{d} \times \mathrm{d}$ block matrix with $r \times r$ blocks $\tilde{\mathbf{a}}_{m}^{[\gamma]}$. Since $\mathrm{P}_{e}$ has compatible symmetry as in (5.8), we have $\left[\mathrm{S}_{m}^{[\gamma]}\right]_{\ell,:}=\varepsilon_{\ell}^{m} \varepsilon_{\ell} z^{k_{\ell}^{m}-k_{\ell}}\left[\mathrm{Sa \tilde {a }}{ }^{[\gamma]}\right]_{\ell,:}$ for $\ell=1, \ldots, r$ and $m=1, \ldots, \mathrm{d}-1$. By (5.6), we have

$$
\left[\mathrm{Sa}_{m}^{[\gamma]}(z)\right]_{\ell, j}=\operatorname{sgn}\left(Q_{\ell, j}^{\gamma}-\gamma\right) \varepsilon_{\ell}^{m} \varepsilon_{j} z^{R_{\ell, j}^{\gamma}+k_{j, \gamma}+k_{\ell}^{m}-k_{\ell}}, \quad \ell, j=1, \ldots, r .
$$

Let $\mathcal{P}:=\mathrm{P}_{e} \mathrm{U}^{\star}:=\sqrt{\mathrm{d}}\left(\mathrm{b}_{m}^{[\gamma]}\right)_{0 \leqslant m, \gamma \leqslant \mathrm{d}-1}$ with $\mathrm{b}_{0}:=\mathrm{a}$. By (5.9) and the definition of $\mathrm{U}^{\star}$ in (5.5), we deduce that

$$
\left[\mathrm{b}_{m}^{[\gamma]}(z)\right]_{\ell, j}=\varepsilon_{\ell}^{m} \varepsilon_{j} z^{R_{\ell, j}^{\gamma}+k_{\ell}^{m}-k_{\ell}}\left[\mathrm{b}_{m}^{\left[Q_{\ell, j}^{\gamma}\right]}\left(z^{-1}\right)\right]_{\ell, j} .
$$

This implies that $\left[\mathrm{Sb}_{m}(z)\right]_{\ell, j}=\varepsilon_{\ell}^{m} \varepsilon_{j} z^{\mathrm{d}\left(k_{\ell}^{m}-k_{\ell}+c_{\ell}\right)-c_{j}}$, which is equivalent to (5.7) with $c_{\ell}^{m}:=k_{\ell}^{m}-k_{\ell}+c_{\ell}$ for $m=1, \ldots, \mathrm{d}-1$ and $\ell=1, \ldots, r$.

Noting that $D_{e}=\operatorname{diag}\left(I_{r}, D_{1}, \ldots, D_{\mathrm{d}-1}\right)$ for some diagonal matrices $D_{m}$ with all the entries of $D_{m} D_{m}^{\star}$ in $\mathbb{F}$, we complete the proof. 
To end this section, for a d-orthogonal wavelet low-pass filter a satisfying (5.1), we provide an algorithm (see Algorithm 3) to construct the associated high-pass filters $b_{1}, \ldots, b_{d-1}$ such that they form a symmetric $d$-orthogonal wavelet filter bank.

$\overline{\text { Algorithm 3. Construction of Wavelet High-Pass Filters with Symmetry Structure }}$

(a) Input. A d-orthogonal wavelet filter $a$ with symmetry as in (5.1) and coefficients in $\mathbb{F}$.

(b) Output. A symmetric d-orthogonal wavelet filter bank $\left\{a, b_{1}, \ldots, b_{\mathrm{d}-1}\right\}$ such that $\mathcal{P}$ in (3.3) is paraunitary and all filters $b_{m}, m=1, \ldots, \mathrm{d}-1$, have symmetry pattern satisfying (5.7). Moreover, $b_{m}=D_{m} \tilde{b}_{m}, m=1, \ldots, \mathrm{d}-1$, where $\tilde{b}_{m}: \mathbb{Z} \rightarrow \mathbb{F}^{r \times r}$ has coefficients from $\mathbb{F}$ and $D_{m}$ is a diagonal matrix with all the entries of $D_{m} D_{m}^{\star}$ in $\mathbb{F}$.

(c) Initialization. Let $\mathrm{P}_{\mathrm{a}}:=\left[\mathrm{a}^{[0]}, \ldots, \mathrm{a}^{[\mathrm{d}-1]}\right]$ and construct $\mathrm{U}$ with respect to (5.5) such that $\mathrm{P}:=\sqrt{\mathrm{d}} \mathrm{P}_{\mathrm{a}} \mathrm{U}=\mathrm{Q}_{0} D_{0}$ for some matrix $\mathrm{Q}_{0}$ of Laurent polynomials in $\mathbb{F}\left[z, z^{-1}\right]$ and some diagonal matrix $D_{0}$ with all the entries of $D_{0} D_{0}^{\star}$ in $\mathbb{F}$. Moreover, $\mathrm{P}$ has compatible symmetry: $\mathrm{SP}(z)=\left[\varepsilon_{1} z^{k_{1}}, \ldots, \varepsilon_{r} z^{k_{r}}\right]^{\top} \mathrm{S} \theta(z)$ for some $k_{1}, \ldots, k_{r} \in \mathbb{Z}$ and some $1 \times \mathrm{d} r$ row vector $\theta$ of Laurent polynomials with symmetry.

(d) Matrix Extension. Derive $\mathrm{P}_{e}$ having all the properties as in Theorem 4.1 from $\mathrm{P}$ by Algorithm 2 ,

(e) High-Pass Filters. Let $\mathcal{P}:=\mathrm{P}_{e} \mathrm{U}^{\star}=: \sqrt{\mathrm{d}}\left(\mathrm{b}_{m}^{[\gamma]}\right)_{0 \leqslant m, \gamma \leqslant \mathrm{d}-1}$ as in (3.3) with $\mathrm{b}_{0}:=\mathrm{a}$. Define high-pass filters $b_{1}, \ldots, b_{\mathrm{d}-1}$ through their symbols by

$$
\mathrm{b}_{m}(z):=\sum_{\gamma=0}^{\mathrm{d}-1} \mathrm{~b}_{m}^{[\gamma]}\left(z^{\mathrm{d}}\right) z^{\gamma}, \quad m=1, \ldots, \mathrm{d}-1 .
$$

\section{Illustrative EXAMPLES}

In this section we provide several examples to illustrate our algorithms and results stated in previous sections.

Example 6.1. Let $\mathrm{d}=3$ and $r=1$. A 3-orthogonal low-pass wavelet filter $a$ is given by its symbol a as follows (also see [2]):

$$
\mathrm{a}(z)=\left(\frac{z^{-1}+1+z}{3}\right)^{2}\left(-\frac{1}{9} z^{-2}-\frac{2}{9} z^{-1}+\frac{5}{3}-\frac{2}{9} z-\frac{1}{9} z^{2}\right) .
$$

All the coefficients of a are rational numbers and the filter $a$ is symmetric about 0 : $\mathrm{Sa}=1$.

Applying Lemma 5.1, we obtain

$\mathrm{P}_{\mathrm{a}}(z):=\left[\mathrm{a}^{[0]}(z), \mathrm{a}^{[1]}(z), \mathrm{a}^{[2]}(z)\right]=\frac{1}{81}\left[-4 z^{-1}+35-4 z, 8 z^{-1}+20-z,-z^{-2}+20 z^{-1}+8\right]$

and $\mathrm{U}$ that symmetrizes $\mathrm{P}_{\mathrm{a}}$ is given by

$$
\mathrm{U}(z)=\left[\begin{array}{ccc}
1 & 0 & 0 \\
0 & \frac{1}{\sqrt{2}} & \frac{1}{\sqrt{2}} \\
0 & \frac{1}{\sqrt{2}} z & -\frac{1}{\sqrt{2}} z
\end{array}\right]
$$

Then $\mathrm{P}:=\sqrt{3} \mathrm{P}_{\mathrm{a}} \mathrm{U}=\mathrm{Q}_{0} D_{0}$ satisfies $\mathrm{SP}=[1,1,-1]$ with $\mathrm{Q}_{0}$ and $D_{0}$ being given by $\mathrm{Q}_{0}(z)=\frac{1}{162}\left[-8 z^{-1}+70-8 z, 7 z^{-1}+40+7 z, 9 z^{-1}-9 z\right], D_{0}=\operatorname{diag}(\sqrt{3}, \sqrt{6}, \sqrt{6})$. 
Applying Algorithm 2 to $\mathrm{P}$, we obtain a desired paraunitary matrix $\mathrm{P}_{e}=$ $D_{e} \mathrm{Q}_{e} D_{0}$ with $D_{e}=\operatorname{diag}\left(\frac{1}{162}, \frac{\sqrt{2}}{324}, \frac{\sqrt{6}}{108}\right)$ and $\mathrm{Q}_{e}$ as follows:

$$
\mathrm{Q}_{e}(z)=\left[\begin{array}{ccc}
-8 z^{-1}+70-8 z & 7 z^{-1}+40+7 z & -9 z^{-1}+9 z \\
40 z^{-1}+28+40 z & -35 z^{-1}+16-35 z & -45 z^{-1}+45 z \\
-8 z^{-1}+8 z & 7 z^{-1}-7 z & 9 z^{-1}+9 z
\end{array}\right]
$$

We have $\mathrm{SP}_{e}=[1,1,-1]^{\top}[1,1,-1]$ and $\operatorname{fsupp}\left(\left[\mathrm{P}_{e}\right]_{:, j}\right) \subseteq \operatorname{fsupp}\left([\mathrm{P}]_{j}\right)$ for all $1 \leqslant j \leqslant 3$. Now, from the polyphase matrix $\mathcal{P}:=\mathrm{P}_{e} \mathrm{U}^{\star}=: \sqrt{3}\left(\mathrm{~b}_{m}^{[\gamma]}\right)_{0 \leqslant m, \gamma \leqslant 2}$ with $\mathrm{b}_{0}:=\mathrm{a}$, we derive two high-pass filters $b_{1}, b_{2}$ as follows:

$$
\begin{aligned}
& \mathrm{b}_{1}(z)=\frac{\sqrt{2}}{162}\left(5 z^{-4}+20 z^{-3}-40 z^{-2}+8 z^{-1}+14+8 z-40 z^{2}+20 z^{3}+5 z^{4}\right), \\
& \mathrm{b}_{2}(z)=\frac{\sqrt{6}}{54}\left(z^{-4}+4 z^{-3}-8 z^{-2}+8 z^{2}-4 z^{3}-z^{4}\right) .
\end{aligned}
$$

The high-pass filter $b_{1}$ is symmetric about $0: S b_{1}=1$, while the high-pass filter $b_{2}$ is antisymmetric about $0: \mathrm{Sb}_{2}=-1$. Note that $\operatorname{len}\left(\mathrm{b}_{1}\right)=\operatorname{len}\left(\mathrm{b}_{2}\right)=\operatorname{len}(\mathrm{a})=8$.

Example 6.2. Let $\mathrm{d}=5$ and $r=1$. A 5 -orthogonal wavelet low-pass filter $a$ is given by its symbol a as follows:

$$
\mathrm{a}(z)=\left(\frac{z^{-2}+z^{-1}+1+z+z^{2}}{5}\right)^{2}\left(-\frac{2}{3} z^{-2}+\frac{2}{3} z^{-1}+1+\frac{2}{3} z-\frac{2}{3} z^{2}\right) .
$$

All the coefficients of a are rational numbers and the filter $a$ is symmetric about 0 : $\mathrm{Sa}=1$.

Applying Lemma [5.1, we obtain

$$
\begin{aligned}
\mathrm{P}_{\mathrm{a}}(z) & :=\left[\mathrm{a}^{[0]}(z), \ldots, \mathrm{a}^{[4]}(z)\right] \\
& =\frac{1}{75}\left[-2 z^{-1}+19-2 z, z^{-1}+16-2 z, 6 z^{-1}+9,9 z^{-1}+6,-2 z^{-2}+16 z^{-1}+1\right]
\end{aligned}
$$

and $\mathrm{U}$ that symmetrizes $\mathrm{P}_{\mathrm{a}}$ is given by

$$
\mathrm{U}(z)=\left[\begin{array}{ccccc}
1 & 0 & 0 & 0 & 0 \\
0 & \frac{1}{\sqrt{2}} & 0 & 0 & \frac{1}{\sqrt{2}} \\
0 & 0 & \frac{1}{\sqrt{2}} & \frac{1}{\sqrt{2}} & 0 \\
0 & 0 & \frac{1}{\sqrt{2}} & -\frac{1}{\sqrt{2}} & 0 \\
0 & \frac{1}{\sqrt{2}} z & 0 & 0 & -\frac{1}{\sqrt{2}} z
\end{array}\right]
$$

Then $\mathrm{P}:=\sqrt{5} \mathrm{P}_{\mathrm{a}} \mathrm{U}=\mathrm{Q}_{0} D_{0}$ satisfies $\mathrm{SP}=\left[1,1, z^{-1},-z^{-1},-1\right]$ with

$$
D_{0}=\operatorname{diag}(\sqrt{5}, \sqrt{10}, \sqrt{10}, \sqrt{10}, \sqrt{10})
$$

and $Q_{0}$ being given by

$$
\mathrm{Q}_{0}(z)=\frac{1}{150}\left[-4 z^{-1}+38-4 z,-z^{-1}+32-z, 15 z^{-1}+15,-3 z^{-1}+3,3 z^{-1}-3 z\right] .
$$

Applying Algorithm 2 to $\mathrm{P}$, we obtain a desired paraunitary matrix $\mathrm{P}_{e}=$ $D_{e} Q_{e} D_{0}$ with $D_{e}=\operatorname{diag}\left(\frac{1}{150}, \frac{1}{300}, \frac{\sqrt{5}}{300}, \frac{\sqrt{5}}{60}, \frac{\sqrt{5}}{300}\right)$ and $\mathrm{Q}_{e}$ as follows:

$$
\mathrm{Q}_{e}(z)=\left[\begin{array}{ccccc}
38-4\left(z^{-1}+z\right) & 32-\left(z^{-1}+z\right) & 15\left(z^{-1}+1\right) & -3\left(z^{-1}+1\right) & 3 z^{-1}-3 z \\
4\left(z^{-1}+z\right)-88 & z^{-1}+68+z & -15 z^{-1}-15 & 3 z^{-1}-3 & -3 z^{-1}+3 z \\
-4 z^{-1}+4 z & -z^{-1}+z & 15 z^{-1}-15 & -3 z^{-1}-3 & 3\left(z^{-1}+1\right)+36 \\
-4-4 z & -1-z & 6 & 0 & 3-3 z \\
-4+4 z & -1+z & 0 & 42 & 3+3 z
\end{array}\right] .
$$


We have $\mathrm{SP}_{e}=[1,1,-1, z,-z]^{\top}\left[1,1, z^{-1},-z^{-1},-1\right]$ and fsupp $\left(\left[\mathrm{P}_{e}\right]_{:, j}\right) \subseteq \operatorname{fsupp}\left([\mathrm{P}]_{j}\right)$ for all $1 \leqslant j \leqslant 5$. Now, from the polyphase matrix $\mathcal{P}:=\mathrm{P}_{e} \mathrm{U}^{\star}=: \sqrt{5}\left(\mathbf{b}_{m}^{[\gamma]}\right)_{0 \leqslant m, \gamma \leqslant 4}$ with $b_{0}:=a$, we derive four high-pass filters $b_{1}, \ldots, b_{4}$ as follows:

$$
\begin{aligned}
& \mathrm{b}_{1}(z)=\frac{1}{150}\left(t_{1}\left(z^{-1}\right)+t_{1}(z)\right) ; \quad \mathrm{b}_{2}(z)=\frac{\sqrt{5}}{150}\left(-t_{2}\left(z^{-1}\right)+t_{2}(z)\right) ; \\
& \mathrm{b}_{3}(z)=\frac{\sqrt{5}}{30}\left(-2 z^{-1}-2+z+3 z^{2}+3 z^{3}+z^{4}-2 z^{5}-2 z^{6}\right) ; \\
& \mathrm{b}_{4}(z)=\frac{\sqrt{5}}{150}\left(-2 z^{-1}-2+z+21 z^{2}-21 z^{3}-z^{4}+2 z^{5}+2 z^{6}\right)
\end{aligned}
$$

with

$t_{1}(z)=-22+34 z-9 z^{2}-6 z^{3}-z^{4}+2 z^{5}+2 z^{6} ; t_{2}(z)=18 z-9 z^{2}-6 z^{3}-z^{4}+2 z^{5}+2 z^{6}$.

The high-pass filter $b_{1}$ is symmetric about $0: S b_{1}=1$, the high-pass filter $b_{2}$ is antisymmetric about $0: \mathrm{Sb}_{2}=-1$, the high-pass filter $\mathrm{b}_{3}$ is symmetric about $5 / 2$ : $\mathrm{Sb}_{3}=z^{5}$, and the high-pass filter $\mathrm{b}_{4}$ is antisymmetric about 5/2: $\mathrm{Sb}_{4}=-z^{5}$. Note that $\operatorname{len}\left(b_{1}\right)=\operatorname{len}\left(b_{2}\right)=\operatorname{len}(a)=12$ and $\operatorname{len}\left(b_{3}\right)=\operatorname{len}\left(b_{4}\right)=7<\operatorname{len}(a)$.

Example 6.3. Let $\mathrm{d}=3$ and $r=1$. A 3-orthogonal low-pass wavelet filter $a$ is given by its symbol a as follows:

$$
\mathrm{a}(z)=\frac{1}{3}\left(\frac{z^{-1}+1+z}{3}\right)^{3}\left(-(3+2 i \sqrt{3}) z^{-1}+9+4 i \sqrt{3}-(3+2 i \sqrt{3}) z\right) .
$$

All the coefficients of a are from the algebraic number field $\mathbb{Q}(i \sqrt{3})$ with $i=\sqrt{-1}$ being the imaginary unit, and the filter $a$ is symmetric about 0 : Sa $=1$.

Applying Lemma 5.1, we obtain

$$
\begin{aligned}
\mathrm{P}_{\mathrm{a}}(z):= & {\left[\mathrm{a}^{[0]}(z), \mathrm{a}^{[1]}(z), \mathrm{a}^{[2]}(z)\right] } \\
= & \frac{1}{81}\left[-2 i \sqrt{3} z^{-1}+(27+4 i \sqrt{3})-2 i \sqrt{3} z,(6-2 i \sqrt{3}) z^{-1}+(24+4 i \sqrt{3})\right. \\
& \left.\quad-(3+2 i \sqrt{3}) z,-(3+2 i \sqrt{3}) z^{-2}+(24+4 i \sqrt{3}) z^{-1}+(6-2 i \sqrt{3})\right]
\end{aligned}
$$

and $\mathrm{U}$ that symmetrizes $\mathrm{P}_{\mathrm{a}}$ is given by

$$
\mathrm{U}(z)=\left[\begin{array}{ccc}
1 & 0 & 0 \\
0 & \frac{1}{\sqrt{2}} & \frac{1}{\sqrt{2}} \\
0 & \frac{1}{\sqrt{2}} z & -\frac{1}{\sqrt{2}} z
\end{array}\right] .
$$

Then $\mathrm{P}:=\sqrt{3} \mathrm{P}_{\mathrm{a}} \mathrm{U}=\mathrm{Q}_{0} D_{0}$ satisfies $\mathrm{SP}=[1,1,-1]$ with $D_{0}=\operatorname{diag}(\sqrt{3}, \sqrt{6}, \sqrt{6})$ and $\mathrm{Q}_{0}$ being given by

$\mathrm{Q}_{0}=\frac{1}{162}\left[-4 i \sqrt{3}\left(z^{-1}+z\right)+(54+8 \sqrt{3} i),(3-4 i \sqrt{3})\left(z^{-1}+z\right)+(48+8 i \sqrt{3}), 9\left(z^{-1}-z\right)\right]$.

Applying Algorithm 2 to $\mathrm{P}$, we obtain a desired paraunitary matrix $\mathrm{P}_{e}=$ $D_{e} Q_{e} D_{0}$ with $D_{e}=\operatorname{diag}\left(\frac{1}{162}, \frac{\sqrt{78}}{4212}, \frac{\sqrt{78}}{1404}\right)$ and $Q_{e}$ as follows:

$$
\mathrm{Q}_{e}(z)=\left[\begin{array}{ccc}
-4 i \sqrt{3}\left(z^{-1}+z\right)+t_{11} & (3-4 i \sqrt{3})\left(z^{-1}+z\right)+t_{12} & 9\left(z^{-1}-z\right) \\
-4\left(z^{-1}+z\right)+t_{21} \sqrt{3} & -(4+i \sqrt{3})\left(z^{-1}+z\right)+t_{22} & 3 i-\sqrt{3}\left(z^{-1}-z\right) \\
-4 i \sqrt{3}\left(z^{-1}-z\right) & (3-4 i \sqrt{3})\left(z^{-1}-z\right) & 9\left(z^{-1}+z\right)-36 i \sqrt{3}
\end{array}\right],
$$

with

$$
t_{11}=54+8 i \sqrt{3} ; t_{12}=48+8 i \sqrt{3} ; t_{21}=-208+36 i \sqrt{3} ; t_{22}=116-16 i \sqrt{3} .
$$


We have $\mathrm{SP}_{e}=[1,1,-1]^{\top}[1,1,-1]$ and $\operatorname{fsupp}\left(\left[\mathrm{P}_{e}\right]_{:, j}\right) \subseteq \operatorname{fsupp}\left([\mathrm{P}]_{j}\right)$ for all $1 \leqslant j \leqslant 3$. Now, from the polyphase matrix $\mathcal{P}:=\mathrm{P}_{e} \mathrm{U}^{\star}=: \sqrt{3}\left(\mathrm{~b}_{m}^{[\gamma]}\right)_{0 \leqslant m, \gamma \leqslant 2}$ with $\mathrm{b}_{0}:=\mathrm{a}$, we derive two high-pass filters $b_{1}, b_{2}$ as follows:

$$
\begin{aligned}
\mathrm{b}_{1}(z)= & \frac{\sqrt{78}}{2106}\left(-(2-i \sqrt{3})\left(z^{-4}+z^{4}\right)-2\left(z^{-3}+z^{3}\right)-(2+2 i \sqrt{3})\left(z^{-2}+z^{2}\right)\right. \\
& \left.+(58-8 i \sqrt{3})\left(z^{-1}+z\right)-104+18 i \sqrt{3}\right), \\
\mathrm{b}_{2}(z)= & \frac{\sqrt{78}}{702}\left(-(3+2 i \sqrt{3})\left(z^{-4}-z^{4}\right)-2 i \sqrt{3}\left(z^{-3}-z^{3}\right)+(6-2 i \sqrt{3})\left(z^{-2}-z^{2}\right)\right. \\
& \left.+18 i \sqrt{3}\left(z^{-1}-z\right)\right)
\end{aligned}
$$

The high-pass filter $b_{1}$ is symmetric about $0: \mathbf{S b}_{1}=1$, while the high-pass filter $b_{2}$ is antisymmetric about $0: \mathrm{Sb}_{2}=-1$. Note that $\operatorname{len}\left(\mathrm{b}_{1}\right)=\operatorname{len}\left(\mathrm{b}_{2}\right)=\operatorname{len}(\mathrm{a})=8$.

Example 6.4. Let $\mathrm{d}=2$ and $r=2$. A 2-orthogonal wavelet low-pass filter a with multiplicity 2 in 4 ] is given by

$$
\mathrm{a}(z)=\frac{1}{40}\left[\begin{array}{cc}
12\left(z^{-1}+1\right) & 16 \sqrt{2} z^{-1} \\
-\sqrt{2}\left(z^{-1}-9-9 z+z^{2}\right) & -2\left(3 z^{-1}-10+3 z\right)
\end{array}\right] .
$$

The low-pass filter a satisfies the symmetry property in (5.1) with $c_{1}=-1, c_{2}=0$ and $\varepsilon_{1}=\varepsilon_{2}=1$. Note that the coefficients of a are from the algebraic number field $\mathbb{F}=\mathbb{Q}(\sqrt{2})$.

Applying Lemma [5.1, we obtain $\mathrm{P}_{\mathrm{a}}:=\left[\mathrm{a}^{[0]}, \mathrm{a}^{[1]}\right]$ and $\mathrm{U}$ as follows:

$$
\mathrm{P}_{\mathrm{a}}(z)=\frac{1}{20}\left[\begin{array}{cc|cc}
6 & 0 & 6 z^{-1} & 8 \sqrt{2} z^{-1} \\
\frac{1}{\sqrt{2}}(9-z) & 10 & \frac{1}{\sqrt{2}}\left(9-z^{-1}\right) & -3\left(1+z^{-1}\right)
\end{array}\right]
$$

and

$$
\mathrm{U}(z)=\frac{1}{\sqrt{2}}\left[\begin{array}{cccc}
1 & 0 & 1 & 0 \\
0 & \sqrt{2} & 0 & 0 \\
z & 0 & -z & 0 \\
0 & 0 & 0 & \sqrt{2} z
\end{array}\right]
$$

Then $\mathrm{P}:=\sqrt{2} \mathrm{P}_{\mathrm{a}} \mathrm{U}$ satisfies $\mathrm{SP}=[1, z]^{\top}\left[1, z^{-1},-1,1\right]=\mathrm{Q}_{0} D_{0}$ with

$$
\mathrm{Q}_{0}(z)=\frac{\sqrt{2}}{20}\left[\begin{array}{cccc}
6 \sqrt{2} & 0 & 0 & 8 \sqrt{2} \\
4(1+z) & 10 & 5(1-z) & -3(1+z)
\end{array}\right], \quad D_{0}=I_{4} .
$$

We would like to point out that though the filter coefficients of $Q_{0}$ are in $\mathbb{Q}(\sqrt{2})$, they are essentially in $\mathbb{Q}$ because $\mathbb{Q}_{0}$ can be written as

$$
\mathrm{Q}_{0}=\frac{1}{20}\left[\begin{array}{cc}
1 & 0 \\
0 & \sqrt{2}
\end{array}\right] \tilde{\mathrm{Q}}_{0} \text { with } \tilde{\mathrm{Q}}_{0}(z)=\left[\begin{array}{cccc}
12 & 0 & 0 & 16 \\
4(1+z) & 10 & 5(1-z) & -3(1+z)
\end{array}\right],
$$

and our algorithms are essentially applied to the part $\tilde{Q}_{0}$.

Applying Algorithm 2 to $\mathrm{P}$, we obtain a desired paraunitary matrix $\mathrm{P}_{e}=D_{e} \mathrm{Q}_{e}$ with $D_{e}$ and $\mathrm{Q}_{e}$ being given as follows:

$$
D_{e}=\operatorname{diag}(2, \sqrt{2}, \sqrt{2}, 2), \quad \mathrm{Q}_{e}(z)=\frac{1}{20}\left[\begin{array}{cccc}
6 & 0 & 0 & 8 \\
4(1+z) & 10 & 5(1-z) & -3(1+z) \\
4(1+z) & -10 & 5(1-z) & -3(1+z) \\
4(1-z) & 0 & 5(1+z) & -3(1-z)
\end{array}\right] \text {. }
$$

We have $\mathrm{SP}_{e}=[1, z, z,-z]^{\top}\left[1, z^{-1},-1,1\right]$ and $\operatorname{fsupp}\left(\left[\mathrm{P}_{e}\right]_{:, j}\right) \subseteq \operatorname{fsupp}\left([\mathrm{P}]_{:, j}\right)$ for all $1 \leqslant j \leqslant 4$. 
Now, from the polyphase matrix $\mathcal{P}:=\mathrm{P}_{e} \mathrm{U}^{\star}=: \sqrt{2}\left(\mathrm{~b}_{m}^{[\gamma]}\right)_{0 \leqslant m, \gamma \leqslant 1}$ with $\mathrm{b}_{0}:=\mathrm{a}$, we derive a high-pass filter $b_{1}$ as follows:

$$
\mathrm{b}_{1}(z)=\frac{1}{40}\left[\begin{array}{cc}
-\sqrt{2}\left(z^{-1}-9-9 z+z^{2}\right) & -2\left(3 z^{-1}+10+3 z\right) \\
2\left(-z^{-1}+9-9 z+z^{2}\right) & -6 \sqrt{2}\left(z^{-1}-z\right)
\end{array}\right] .
$$

Then the high-pass filter $\mathrm{b}_{1}$ satisfies (5.7) with $c_{1}^{1}=c_{2}^{1}=0$ and $\varepsilon_{1}^{1}=1, \varepsilon_{2}^{1}=-1$.

Our algorithms are applicable to many other examples with $\mathrm{d} \geqslant 2$ and $r \in \mathbb{N}$; for example, see [10, Examples 2 and 3] and many examples in [5, 8].

\section{Proofs of Lemmas 4.2 and 4.3}

We now prove Lemmas 4.2 and 4.3 stated in Section 4 . To do so, we need an auxiliary result. The following lemma shows that for a $1 \times 4$ vector $\mathrm{p}$ of Laurent polynomials with symmetry such that $\mathrm{p}(z) \mathrm{p}^{\star}(z)=1$ for all $z \in \mathbb{C} \backslash\{0\}$, Sp $=$ $\left[1,-1, z^{-1},-z^{-1}\right]$, and $\mathrm{p}(z)=\mathrm{q}_{0}(z) D_{0}$, we can construct a $4 \times 4$ paraunitary matrix with a special structure.

Lemma 7.1. Let $\mathrm{p}$ be $a 1 \times 4$ row vector of Laurent polynomials with symmetry satisfying $\mathrm{p}(z) \mathrm{p}^{\star}(z)=1$ for all $z \in \mathbb{C} \backslash\{0\}, \operatorname{Sp}(z)=\left[1,-1, z^{-1},-z^{-1}\right]$, and $\mathrm{p}(z)=\mathrm{q}_{0}(z) D_{0}$ for some $1 \times 4$ vector $\mathrm{q}_{0}$ of Laurent polynomials in $\mathbb{F}\left[z, z^{-1}\right]$ and some diagonal matrix $D_{0}=\operatorname{diag}\left(d_{1}, d_{1}, d_{3}, d_{4}\right)$, where $d_{1}, d_{3}$, and $d_{4}$ are positive numbers with $d_{1}^{2}, d_{3}^{2}$, and $d_{4}^{2}$ being numbers in $\mathbb{F}$. Suppose $\operatorname{fsupp}(\mathrm{p})=[-k, k]$ with a positive integer $k \geqslant 1, \operatorname{coeff}(\mathbf{q},-k)=\left[f_{1},-f_{1}, g_{1},-g_{2}\right]$, and $\operatorname{coeff}(\mathbf{q},-k+1)=$ $\left[f_{3},-f_{4}, g_{3},-g_{4}\right]$ for some numbers $f_{1}, f_{3}, f_{4}, g_{1}, g_{2}, g_{3}, g_{4}$ in $\mathbb{F}$ with $f_{1}>0$ and $g_{1}, g_{2} \geqslant 0$, i.e., $\mathrm{q}_{0}^{\top}$ takes the form:

$$
\mathrm{q}_{0}(z)^{\top}=\left[\begin{array}{c}
f_{1} \\
-f_{1} \\
g_{1} \\
-g_{2}
\end{array}\right] z^{-k}+\left[\begin{array}{c}
f_{3} \\
-f_{4} \\
g_{3} \\
-g_{4}
\end{array}\right] z^{-k+1}+\cdots+\left[\begin{array}{c}
f_{3} \\
f_{4} \\
g_{1} \\
g_{2}
\end{array}\right] z^{k-1}+\left[\begin{array}{c}
f_{1} \\
f_{1} \\
0 \\
0
\end{array}\right] z^{k} .
$$

Define a matrix $\mathrm{U}_{0}(z):=D_{0}^{\star} \mathrm{V}_{0}(z) D_{1}$ with $D_{1}=\frac{1}{c} \operatorname{diag}\left(1,1, \frac{d_{3}}{d_{1}}, \frac{d_{4}}{d_{1}}\right)$ and $\mathrm{V}_{0}$ being given by

$$
\mathrm{V}_{0}(z):=\left[\begin{array}{cccc}
f_{1}\left(z+z^{-1}\right)+\bar{f} & f_{1}\left(z-z^{-1}\right) & g_{1}\left(1+z^{-1}\right) & g_{2}\left(1-z^{-1}\right) \\
-f_{1}\left(z-z^{-1}\right) & -f_{1}\left(z+z^{-1}\right)+\bar{f} & -g_{1}\left(1-z^{-1}\right) & -g_{2}\left(1+z^{-1}\right) \\
g_{1}(1+z) & -g_{1}(1-z) & -\frac{d_{1}^{2}}{d_{3}^{2}}\left(2 f_{1}+f\right) & 0 \\
g_{2}(1-z) & -g_{2}(1+z) & 0 & \frac{d_{1}^{2}}{d_{4}^{2}}\left(2 f_{1}-f\right)
\end{array}\right],
$$

where $f:=f_{3}-f_{4}$ and $c:=\sqrt{4 d_{1}^{2} f_{1}^{2}+2 d_{3}^{2} g_{1}^{2}+2 d_{4}^{2} g_{2}^{2}+d_{1}^{2}|f|^{2}}$. Then $\mathrm{U}_{0}$ is paraunitary and has the following properties:

(i) $\operatorname{fsupp}\left(\mathrm{U}_{0}\right)=[-1,1]$ and $\mathrm{SU}_{0}(z)=[1,-1, z,-z]^{\top}\left[1,-1, z^{-1},-z^{-1}\right]$; that is, $\mathrm{U}_{0}$ has compatible symmetry with filter support on $[-1,1]$;

(ii) $\operatorname{fsupp}\left(\mathrm{pU}_{0}\right)=[-k+1, k-1]$ and $\mathrm{S}\left(\mathrm{qU}_{0}\right)=\mathrm{S}(\mathrm{p})$; that is, $\mathrm{U}_{0}$ reduces the length of $\mathrm{p}$ exactly by 2 and preserves the symmetry pattern of $\mathrm{p}$;

(iii) for any vector $\tilde{\mathrm{p}}$ of Laurent polynomials such that $\tilde{\mathrm{p}} \mathrm{p}^{\star}=0$ and $\mathrm{S} \tilde{\mathrm{p}}(z)=$ $\epsilon z^{k_{0}} \mathrm{Sp}(z)$ for some $\epsilon \in\{-1,1\}$ and some $k_{0} \in \mathbb{Z}$, we have $\mathrm{S}\left(\tilde{\mathrm{p}} \mathrm{U}_{0}\right)=\mathrm{S} \tilde{\mathrm{p}}$ and $\operatorname{fsupp}\left(\tilde{\mathrm{p}} \mathrm{U}_{0}\right) \subseteq \operatorname{fsupp}(\tilde{\mathrm{p}}) ;$ that is, $\mathrm{U}_{0}$ keeps the symmetry pattern of $\tilde{\mathrm{p}}$ and does not increase the length of $\tilde{\mathrm{p}}$. 
Proof. Note that $\mathrm{p}$ is of the form:

$$
\mathrm{p}(z)^{\top}=D_{0}^{\top}\left(\left[\begin{array}{c}
f_{1} \\
-f_{1} \\
g_{1} \\
-g_{2}
\end{array}\right] z^{-k}+\left[\begin{array}{c}
f_{3} \\
-f_{4} \\
g_{3} \\
-g_{4}
\end{array}\right] z^{-k+1}+\cdots+\left[\begin{array}{c}
f_{3} \\
f_{4} \\
g_{1} \\
g_{2}
\end{array}\right] z^{k-1}+\left[\begin{array}{c}
f_{1} \\
f_{1} \\
0 \\
0
\end{array}\right] z^{k}\right) .
$$

For such a vector $\mathrm{p}$, by [6], a paraunitary matrix, that reduces its length by 2 and preserves its symmetry pattern, is given by

$$
\mathrm{U}_{0}(z)=\frac{1}{c}\left[\begin{array}{cccc}
d_{1} f_{1}\left(z+z^{-1}\right)+d_{1} \bar{f} & d_{1} f_{1}\left(z-z^{-1}\right) & d_{3} g_{1}\left(1+z^{-1}\right) & d_{4} g_{2}\left(1-z^{-1}\right) \\
-d_{1} f_{1}\left(z-z^{-1}\right) & -d_{1} f_{1}\left(z+z^{-1}\right)+d_{1} \bar{f} & -d_{3} g_{1}\left(1-z^{-1}\right) & -d_{4} g_{2}\left(1+z^{-1}\right) \\
d_{3} g_{1}(1+z) & -d_{3} g_{1}(1-z) & -2 d_{1} f_{1}-d_{1} f & 0 \\
d_{4} g_{2}(1-z) & -d_{4} g_{2}(1+z) & 0 & 2 d_{1} f_{1}-d_{1} f
\end{array}\right]
$$

with $c=\sqrt{4 d_{1}^{2} f_{1}^{2}+2 d_{3}^{2} g_{1}^{2}+2 d_{4}^{2} g_{2}^{2}+d_{1}^{2}|f|^{2}}$ and $f=f_{3}-f_{4}$. It is easy to check that $\mathrm{U}_{0}$ can be rewritten as in the lemma. The properties of $\mathrm{U}_{0}$ in items (i) - (iii) can be verified directly by computations.

We remark that the above result holds even when $[\mathrm{p}]_{3}$ or $[\mathrm{p}]_{4}$ is empty, or both $[\mathrm{p}]_{3}$ and $[\mathrm{p}]_{4}$ are empty. In such a case, we obtain either a $2 \times 2$ or a $3 \times 3$ paraunitary matrix by simply deleting the corresponding row(s) and column(s) in (7.2).

Thanks to Lemmas 2.2 and 7.1, we now prove Lemma 4.2 as follows.

Proof of Lemma 4.2. The paraunitary matrix $\mathrm{B}_{\mathrm{p}}$ is constructed according to the following steps: first reduce the problem size to a $1 \times 4$ vector of Laurent polynomials, then construct a $4 \times 4$ paraunitary matrix according to Lemma 7.1 and finally extend it in a simple way to an $s \times s$ paraunitary matrix.

(1) Let $\mathrm{p}_{0}:=\mathrm{pU}_{\mathrm{Sp}}$, where $\mathrm{U}_{\mathrm{Sp}}$ is a matrix defined in (4.7). Then

$$
\mathrm{Sp}_{0}=\left[\mathbf{1}_{s_{1}},-\mathbf{1}_{s_{2}}, z^{-1} \mathbf{1}_{s_{3}},-z^{-1} \mathbf{1}_{s_{4}}\right]
$$

with $s_{1}, \ldots, s_{4}$ being nonnegative integers determined by $\mathrm{Sp} . \mathrm{p}_{0}$ must take one of the following forms:

$$
\begin{aligned}
\mathrm{p}_{0}= & \left(\left[\mathrm{f}_{1},-\mathrm{f}_{2}, \mathrm{~g}_{1},-\mathrm{g}_{2}\right] z^{\ell_{1}}+\left[\mathrm{f}_{3},-\mathrm{f}_{4}, \mathrm{~g}_{3},-\mathrm{g}_{4}\right] z^{\ell_{1}+1}+\sum_{\ell=\ell_{1}+2}^{\ell_{2}-2} \operatorname{coeff}\left(\mathrm{p}_{0}, \ell\right) z^{\ell}\right. \\
& \left.+\left[\mathrm{f}_{3}, \mathrm{f}_{4}, \mathrm{~g}_{1}, \mathrm{~g}_{2}\right] z^{\ell_{2}-1}+\left[\mathrm{f}_{1}, \mathrm{f}_{2}, \mathbf{0}, \mathbf{0}\right] z^{\ell_{2}}\right) \tilde{D}_{0} ; \\
\mathrm{p}_{0}= & \left(\left[\mathbf{0}, \mathbf{0}, \mathrm{f}_{1},-\mathrm{f}_{2}\right] z^{\ell_{1}}+\left[\mathrm{g}_{1},-\mathrm{g}_{2}, \mathrm{f}_{3},-\mathrm{f}_{4}\right] z^{\ell_{1}+1}+\sum_{\ell=\ell_{1}+2}^{\ell_{2}-2} \operatorname{coeff}\left(\mathrm{p}_{0}, \ell\right) z^{\ell}\right. \\
& \left.+\left[\mathrm{g}_{3}, \mathrm{~g}_{4}, \mathrm{f}_{3}, \mathrm{f}_{4}\right] z^{\ell_{2}-1}+\left[\mathrm{g}_{1}, \mathrm{~g}_{2}, \mathrm{f}_{1}, \mathrm{f}_{2}\right] z^{\ell_{2}}\right) \tilde{D}_{0} .
\end{aligned}
$$

If $\mathrm{p}_{0}$ is of the form (7.3), we simply let $\mathrm{D}_{\mathrm{p}}:=\mathrm{U}_{\mathrm{Sp}}$. If $\mathrm{p}_{0}$ takes the form in (7.4), we further construct a permutation matrix $E$ such that $\left[\mathrm{g}_{1}, \mathrm{~g}_{2}, \mathrm{f}_{1}, \mathrm{f}_{2}\right] E=\left[\mathrm{f}_{1}, \mathrm{f}_{2}, \mathrm{~g}_{1}, \mathrm{~g}_{2}\right]$ and define $\mathrm{D}_{\mathrm{p}}(z):=\mathrm{U}_{\mathrm{Sp}} E \operatorname{diag}\left(I_{s-s_{\mathrm{g}}}, z^{-1} I_{s_{\mathrm{g}}}\right)$, where $1 \times s_{\mathrm{g}}$ is the size of the row vector $\left[\mathrm{g}_{1}, \mathrm{~g}_{2}\right]$. In this way, $\mathrm{p}_{0}:=\mathrm{pD}_{\mathrm{p}}$ always takes the form in $(7.3)$ with $\mathrm{f}_{1} \neq \mathbf{0}$. Moreover, the diagonal matrix $\tilde{D}_{0}$ is given by $\tilde{D}_{0}=\mathrm{D}_{\mathrm{p}}^{\star} D_{0} \mathrm{D}_{\mathrm{p}}=: \operatorname{diag}\left(\tilde{D}_{0,1}, \tilde{D}_{0,2}, \tilde{D}_{0,3}, \tilde{D}_{0,4}\right)$, where $\tilde{D}_{0,1}, \tilde{D}_{0,2}$, $\tilde{D}_{0,3}, \tilde{D}_{0,4}$ are of the same sizes with respect to the vectors $f_{1}, f_{2}, g_{1}, g_{2}$. Define $\tilde{\mathrm{f}}_{1}:=\mathrm{f}_{1} \tilde{D}_{0,1}, \tilde{\mathrm{f}}_{2}:=\mathrm{f}_{2} \tilde{D}_{0,2}, \tilde{\mathrm{g}}_{1}:=\mathrm{g}_{1} \tilde{D}_{0,3}$, and $\tilde{\mathrm{g}}_{2}:=\mathrm{g}_{2} \tilde{D}_{0,4}$. 
(2) Applying Lemma 2.2 to the vectors $\tilde{\mathrm{f}}_{1}, \tilde{\mathrm{f}}_{2}, \tilde{\mathrm{g}}_{1}, \tilde{\mathrm{g}}_{2}$, we can construct unitary constant matrices $U_{\tilde{\mathrm{f}}_{1}}=\tilde{D}_{0,1}^{\star} V_{\tilde{\mathrm{f}}_{1}} \tilde{D}_{1,1}, U_{\tilde{\mathrm{f}}_{2}}=\tilde{D}_{0,2}^{\star} V_{\tilde{\mathrm{f}}_{2}} \tilde{D}_{1,2}, U_{\tilde{\mathrm{g}}_{1}}=$ $\tilde{D}_{0,3}^{\star} V_{\tilde{\mathrm{g}}_{1}} \tilde{D}_{1,3}$, and $U_{\tilde{\mathrm{g}}_{2}}=\tilde{D}_{0,4}^{\star} V_{\tilde{\mathrm{g}}_{2}} \tilde{D}_{1,4}$, where $\tilde{V}_{\tilde{\mathrm{f}}_{1}}=\left[\mathrm{f}_{1}^{\star}, F_{1}^{\star}\right], \tilde{V}_{\tilde{\mathrm{f}}_{2}}=\left[\mathrm{f}_{2}^{\star}, F_{2}^{\star}\right]$, $\tilde{V}_{\tilde{\mathrm{g}}_{1}}=\left[\mathrm{g}_{1}^{\star}, G_{1}^{\star}\right]$, and $\tilde{V}_{\tilde{\mathrm{g}}_{2}}=\left[\mathrm{g}_{2}^{\star}, G_{2}^{\star}\right]$ are nonsingular matrices with all the entries in $\mathbb{F}$. Moreover, $U:=\operatorname{diag}\left(U_{\mathrm{f}_{1}}, U_{\mathrm{f}_{2}}, U_{\mathrm{g}_{1}}, U_{\mathrm{g}_{2}}\right)=\tilde{D}_{0}^{\star} V \tilde{D}_{1}$ with $\tilde{D}_{1}:=$ $\operatorname{diag}\left(\tilde{D}_{1,1}, \tilde{D}_{1,2}, \tilde{D}_{1,3}, \tilde{D}_{1,4}\right)$ normalizes the vector $\left[\mathrm{f}_{1},-\mathrm{f}_{2}, \mathrm{~g}_{1},-\mathrm{g}_{2}\right] \tilde{D}_{0}$ to the following form:

$$
\begin{aligned}
& {\left[\mathrm{f}_{1},-\mathrm{f}_{2}, \mathrm{~g}_{1},-\mathrm{g}_{2}\right] \tilde{D}_{0} U} \\
& \quad=\left[d_{1} f_{1}, 0, \ldots, 0,-d_{1} f_{1}, 0, \ldots, 0, d_{3} g_{1}, 0, \ldots, 0,-d_{4} g_{2}, 0, \ldots, 0\right],
\end{aligned}
$$

where we can choose $d_{1}, d_{3}, d_{4}$ and $f_{1}, g_{1}, g_{2}$ to be $d_{1}=\left\|\tilde{\mathbf{f}}_{1}\right\|, d_{3}=\left\|\tilde{\mathrm{g}}_{1}\right\|$, $d_{4}=\left\|\tilde{\mathrm{g}}_{2}\right\|$ and $f_{1}=g_{1}=g_{2}=1$ (other choices are possible as long as $d_{1}, d_{3}, d_{4}$ and $f_{1}, g_{1}, g_{2}$ satisfy conditions in Lemma 7.1). In other words, under the action of $U, \tilde{\mathbf{f}}_{1}, \tilde{\mathbf{f}}_{2}, \tilde{\mathrm{g}}_{1}$, and $\tilde{\mathrm{g}}_{2}$ become $\left[d_{1} f_{1}, \mathbf{0}\right],\left[d_{1} f_{1}, \mathbf{0}\right],\left[d_{3} g_{1}, \mathbf{0}\right]$, and $\left[d_{4} g_{2}, \mathbf{0}\right]$, respectively. Note that $\tilde{\mathbf{f}}_{1} U_{\tilde{\mathbf{f}}_{1}}=\tilde{\mathbf{f}}_{2} U_{\tilde{\mathbf{f}}_{2}}=\left[d_{1} f_{1}, 0, \ldots, 0\right]$ follows from the fact that $\left\|\tilde{\mathbf{f}}_{1}\right\|=\left\|\tilde{\mathbf{f}}_{2}\right\|$, and $d_{3} g_{1}$ or $d_{4} g_{2}$ could be an empty entry.

(3) Applying Lemma 7.1 to the $1 \times 4$ subvector of $\mathrm{p}_{0} U=\mathrm{pD}_{\mathrm{p}} U$ consisting its 1st, $\left(1+s_{1}\right)$ th, $\left(1-\delta\left(s_{3}\right)\right)\left(1+s_{1}+s_{2}\right)$ th, and $\left(1-\delta\left(s_{4}\right)\right)\left(1+s_{1}+s_{2}+s_{3}\right)$ th entries, we obtain a paraunitary matrix $U_{0}$ as described in Lemma 7.1 Note that $\mathrm{U}_{0}$ could be a $2 \times 2$ or $3 \times 3$ matrix depending on whether or not $d_{3} g_{1}$ or $d_{4} g_{2}$ is an empty entry. Let $\mathcal{I}:=\left\{1,1+s_{1},\left(1-\delta\left(s_{3}\right)\right)\left(1+s_{1}+\right.\right.$ $\left.\left.s_{2}\right),\left(1-\delta\left(s_{4}\right)\right)\left(1+s_{1}+s_{2}+s_{3}\right)\right\} \backslash\{0\}$ be an index set and $n_{0}:=\#(\mathcal{I})$ be its cardinality.

(4) Extend $\mathrm{U}_{0}$ to an $s \times s$ paraunitary matrix $\mathrm{U}$ as follows:

$$
[\mathrm{U}]_{\mathcal{I}_{j}, \mathcal{I}_{k}}:=\left[\mathrm{U}_{0}\right]_{j, k}, j, k=1, \ldots, n_{0} ; \quad[\mathrm{U}]_{j, j}:=1, j \notin \mathcal{I},
$$

and all other entries of $\mathrm{U}$ are zero. Then $\mathrm{U}$ is of the form $\mathrm{U}=\tilde{D}_{2}^{\star} \mathrm{V}_{2} \tilde{D}_{3}$ for some strongly invertible matrix $\mathrm{V}_{2}$ extended from (7.1), where

$$
\tilde{D}_{2}=\operatorname{diag}\left(d_{1}, \mathbf{1}_{s_{1}-1}, d_{1}, \mathbf{1}_{s_{2}-1}, d_{3}, \mathbf{1}_{s_{3}-1}, d_{4}, \mathbf{1}_{s_{4}-1}\right)
$$

and

$$
\tilde{D}_{3}=\operatorname{diag}\left(\frac{1}{c}, \mathbf{1}_{s_{1}-1}, \frac{1}{c}, \mathbf{1}_{s_{2}-1}, \frac{d_{3}}{d_{1} c}, \mathbf{1}_{s_{3}-1}, \frac{d_{4}}{d_{1} c}, \mathbf{1}_{s_{4}-1}\right)
$$

with $c$ defined in (7.1).

(5) Define $\mathrm{B}_{\mathrm{p}_{0}}(z):=U \mathrm{UU}(z)=\left(\tilde{D}_{0}^{\star} V \tilde{D}_{1}\right)\left(\tilde{D}_{2}^{\star} \bigvee_{1} \tilde{D}_{3}\right)$. Then $\mathrm{B}_{\mathrm{p}_{0}}$ satisfies item (i) - (iv) of Lemma 4.2 for $\mathrm{p}_{0}$, and by our construction, $\mathrm{B}_{\mathrm{p}_{0}}$ is the form $\mathrm{B}_{\mathrm{p}_{0}}=\tilde{D}_{0}^{\star} \mathrm{V}_{0} \tilde{D}_{4}$ :

$\mathrm{V}_{0}^{\star}(z):=\left[\begin{array}{c|c|c|c}\mathrm{f}_{1}\left(z+c_{0}+\frac{1}{z}\right) & \mathrm{f}_{2}\left(z-\frac{1}{z}\right) & \mathrm{g}_{1}\left(1+\frac{1}{z}\right) & \mathrm{g}_{2}\left(1-\frac{1}{z}\right) \\ \hline F_{1} & \mathbf{0} & \mathbf{0} & \mathbf{0} \\ \hline-\mathrm{f}_{1}\left(z-\frac{1}{z}\right) & -\mathrm{f}_{2}\left(z-c_{0}+\frac{1}{z}\right) & -\mathrm{g}_{1}\left(1-\frac{1}{z}\right) & -\mathrm{g}_{2}\left(1+\frac{1}{z}\right) \\ \mathbf{0} & F_{2} & \mathbf{0} & \mathbf{0} \\ \hline \mathrm{f}_{1}(1+z) & -\mathrm{f}_{2}(1-z) & \mathrm{g}_{1} & \mathbf{0} \\ \mathbf{0} & \mathbf{0} & G_{1} & \mathbf{0} \\ \hline \mathrm{f}_{1}(1-z) & \mathrm{f}_{2}(1+z) & \mathbf{0} & \mathrm{g}_{2} \\ \mathbf{0} & \mathbf{0} & \mathbf{0} & G_{2}\end{array}\right]$


where $c_{0}=\frac{1}{\left\|\tilde{f}_{1}\right\|^{2}} \operatorname{coeff}\left(\mathrm{p}_{0}, \ell_{1}+1\right) \operatorname{coeff}\left(\mathrm{p}_{0}^{\star},-\ell_{2}\right) \in \mathbb{F}$ and $\tilde{D}_{4}$ is a diagonal matrix determined by $\tilde{D}_{1}, \tilde{D}_{2}$ and $\tilde{D}_{3}$.

(6) Define $\mathrm{B}_{\mathrm{p}}(z):=\mathrm{D}_{\mathrm{p}}(z) \mathrm{B}_{\mathrm{p}_{0}}(z) \mathrm{D}_{\mathrm{p}}^{\star}(z)$. Then $\mathrm{B}_{\mathrm{p}}$ is a desired paraunitary matrix.

Properties (i) - (v) of $B_{p}$ follow from our construction.

We complete the paper by proving Lemma 4.3 .

Proof of Lemma 4.3. The proof is similar to that of Lemma4.2. First, one can show that there exists a permutation matrix $E_{\left(\mathbf{q}_{1}, \mathbf{q}_{2}\right)}$ such that $\mathbf{q}_{1} E_{\left(\mathbf{q}_{1}, \mathbf{q}_{2}\right)}$ and $\mathbf{q}_{2} E_{\left(\mathbf{q}_{1}, \mathbf{q}_{2}\right)}$ take the following form:

$$
\begin{aligned}
{\left[\begin{array}{l}
\mathrm{p}_{1} \\
\mathrm{p}_{2}
\end{array}\right]:=} & {\left[\begin{array}{l}
\mathrm{q}_{1} \\
\mathrm{q}_{2}
\end{array}\right] E_{\left(\mathrm{q}_{1}, \mathrm{q}_{2}\right)}=\left[\begin{array}{cccc}
\mathbf{0} & \mathbf{0} & \tilde{\mathrm{g}}_{3} & -\tilde{\mathrm{g}}_{4} \\
\hline \mathbf{0} & \mathbf{0} & \mathbf{0} & \mathbf{0}
\end{array}\right] z^{-k}+\left[\begin{array}{cccc}
\tilde{\mathrm{f}}_{5} & -\tilde{\mathbf{f}}_{6} & \tilde{\mathrm{g}}_{7} & -\tilde{\mathrm{g}}_{8} \\
\hline \tilde{\mathrm{g}}_{1} & -\tilde{\mathrm{g}}_{2} & \tilde{\mathrm{f}}_{7} & -\tilde{\mathrm{f}}_{8}
\end{array}\right] z^{-k+1} } \\
& +\cdots+\left[\begin{array}{cccc}
\tilde{\mathbf{f}}_{5} & \tilde{\mathrm{f}}_{6} & \tilde{\mathrm{g}}_{3} & \tilde{\mathrm{g}}_{4} \\
\hline \tilde{\mathrm{g}}_{5} & \tilde{\mathrm{g}}_{6} & \tilde{\mathbf{f}}_{7} & \tilde{\mathbf{f}}_{8}
\end{array}\right] z^{k-1}+\left[\begin{array}{cccc}
\mathbf{0} & \mathbf{0} & \mathbf{0} & \mathbf{0} \\
\hline \tilde{\mathrm{g}}_{1} & \tilde{\mathrm{g}}_{2} & \mathbf{0} & \mathbf{0}
\end{array}\right] z^{k}
\end{aligned}
$$

where $\tilde{\mathrm{g}}_{1}, \tilde{\mathrm{g}}_{2}, \tilde{\mathrm{g}}_{3}, \tilde{\mathrm{g}}_{4}$ are all nonzero row vectors of size $1 \times s_{1}, 1 \times s_{2}, 1 \times s_{3}, 1 \times s_{4}$, respectively. And $\left[\tilde{\mathrm{g}}_{1}, \tilde{\mathrm{g}}_{2}, \tilde{\mathrm{g}}_{3}, \tilde{\mathrm{g}}_{4}\right]=\left[\mathrm{g}_{1}, \mathrm{~g}_{2}, \mathrm{~g}_{3}, \mathrm{~g}_{4}\right] \tilde{D}_{0}$ for some vectors $\mathrm{g}_{1}, \ldots, \mathrm{g}_{4}$ with all their entries in $\mathbb{F}$ and having same size as $\tilde{\mathrm{g}}_{1}, \ldots, \tilde{\mathrm{g}}_{4}$, respectively, and for some diagonal matrix $\tilde{D}_{0}$ with all the entries of $\tilde{D}_{0} \tilde{D}_{0}^{\star}$ in $\mathbb{F}$. Note that $\left\|\tilde{\mathrm{g}}_{1}\right\|=\left\|\tilde{\mathrm{g}}_{2}\right\|$ and $\left\|\tilde{\mathrm{g}}_{3}\right\|=\left\|\tilde{\mathrm{g}}_{4}\right\|$.

Using Lemma 2.2. we can construct unitary matrices $U_{\tilde{\mathrm{g}}_{1}}, U_{\tilde{\mathrm{g}}_{2}}, U_{\tilde{\mathrm{g}}_{2}}, U_{\tilde{\mathrm{g}}_{4}}$ of the form $U_{\tilde{\mathrm{g}}_{1}}=\tilde{D}_{0,1}^{\star}\left[\mathrm{g}_{1}^{\star}, G_{1}^{\star}\right] \tilde{D}_{1,1}^{\star}, U_{\tilde{\mathrm{g}}_{2}}=\tilde{D}_{0,2}^{\star}\left[\mathrm{g}_{1}^{\star}, G_{2}^{\star}\right] \tilde{D}_{1,2}^{\star}, U_{\tilde{\mathrm{g}}_{3}}=\tilde{D}_{0,3}^{\star}\left[\mathrm{g}_{3}^{\star}, G_{3}^{\star}\right] \tilde{D}_{1,3}^{\star}$, and $U_{\tilde{\mathrm{g}}_{4}}=\tilde{D}_{0,4}^{\star}\left[\mathrm{g}_{1}^{\star}, G_{4}^{\star}\right] \tilde{D}_{1,4}^{\star}$, where $\tilde{D}_{0}=: \operatorname{diag}\left(\tilde{D}_{0,1}, \tilde{D}_{0,2}, \tilde{D}_{0,3}, \tilde{D}_{0,4}\right), G_{1}, \ldots, G_{4}$ are nonsingular matrices with all the entries in $\mathbb{F}$, and $\tilde{D}_{1}:=\operatorname{diag}\left(\tilde{D}_{1,1}, \tilde{D}_{1,2}, \tilde{D}_{1,3}, \tilde{D}_{1,4}\right)$ is some diagonal matrix with all the entries of $\tilde{D}_{1} \tilde{D}_{1}^{\star}$ in $\mathbb{F}$.

Define $U:=\operatorname{diag}\left(U_{\tilde{\mathrm{g}}_{1}}, U_{\tilde{\mathrm{g}}_{2}}, U_{\tilde{\mathrm{g}}_{2}}, U_{\tilde{\mathrm{g}}_{4}}\right)=: \tilde{D}_{0} V \tilde{D}_{1}$. Applying $U$ to the pair $\left(\mathrm{p}_{1}, \mathrm{p}_{2}\right)$, we normalize the pair to be supported on $[-k+1, k-1]$ except those entries on 1st, $\left(1+s_{1}\right)$ th, $\left(1+s_{1}+s_{2}\right)$ th, and $\left(1+s_{1}+s_{2}+s_{3}\right)$ th positions. Hence, we only need to consider a problem of constructing a $4 \times 4$ paraunitary matrix $\mathrm{U}_{0}$, which can be constructed by Lemma 7.1 similarly as in the proof Lemma 4.2 . corresponding to the $1 \times 4$ vector of Laurent polynomials with symmetry. Finally, we can extend $\mathrm{U}_{0}$ into a full $s \times s$ paraunitary matrix $\mathrm{U}$ of the form $\mathrm{U}=\tilde{D}_{2} \mathrm{\vee}_{1} \tilde{D}_{3}$. Consequently, the paraunitary matrix for the pair $\left(\mathrm{p}_{1}, \mathrm{p}_{2}\right)$ is given by $\mathrm{B}_{\left(\mathrm{p}_{1}, \mathrm{p}_{2}\right)}=$ $U \mathrm{U}=\left(\tilde{D}_{0}^{\star} V \tilde{D}_{1}\right)\left(\tilde{D}_{2}^{\star} \mathrm{\vee}_{1} \tilde{D}_{3}\right)$, and by our construction, $\mathrm{B}_{\left(\mathrm{p}_{1}, \mathrm{p}_{2}\right)}$ can be written as $\mathrm{B}_{\left(\mathrm{p}_{1}, \mathrm{p}_{2}\right)}=\tilde{D}_{0} \mathrm{\vee}_{0} \tilde{D}_{4}$ with $\mathrm{\vee}_{0}$ being given as follows:

$$
\mathrm{V}_{0}^{\star}(z):=\left[\begin{array}{c|c|c|c}
\mathrm{g}_{1} & \mathbf{0} & \mathrm{g}_{3}\left(1+\frac{1}{z}\right) & \mathrm{g}_{4}\left(1-\frac{1}{z}\right) \\
G_{1} & \mathbf{0} & \mathbf{0} & \mathbf{0} \\
\hline \mathbf{0} & \mathrm{g}_{2} & -\mathrm{g}_{3}\left(1-\frac{1}{z}\right) & -\mathrm{g}_{4}\left(1+\frac{1}{z}\right) \\
\mathbf{0} & G_{2} & \mathbf{0} & \mathbf{0} \\
\hline \mathrm{g}_{1}(1+z) & -\mathrm{g}_{2}(1-z) & -\mathrm{g}_{3} & \mathbf{0} \\
\mathbf{0} & \mathbf{0} & G_{3} & \mathbf{0} \\
\hline \mathrm{g}_{1}(1-z) & -\mathrm{g}_{2}(1+z) & \mathbf{0} & -\mathrm{g}_{4} \\
\mathbf{0} & \mathbf{0} & \mathbf{0} & G_{4}
\end{array}\right] \text {, }
$$


and $\tilde{D}_{4}$ being determined by $\tilde{D}_{1}, \tilde{D}_{2}$, and $\tilde{D}_{3}$. Let $\mathrm{B}_{\left(\mathrm{q}_{1}, \mathrm{q}_{2}\right)}:=E_{\left(\mathbf{q}_{1}, \mathrm{q}_{2}\right)} \mathrm{B}_{\left(\mathrm{p}_{1}, \mathrm{p}_{2}\right)} E_{\left(\mathbf{q}_{1}, \mathbf{q}_{2}\right)}^{\top}$. Then $B_{\left(q_{1}, q_{2}\right)}$ is a desired matrix and properties (i) - (v) follow from our construction.

\section{ACKNOWLEDGMENTS}

We would like to thank the anonymous reviewers for suggesting many helpful improvements to this paper.

\section{REFERENCES}

1. N. Bi, B. Han, and Z. Shen, Examples of refinable componentwise polynomials, Appl. Comput. Harmon. Anal., 22 (2007), 368-373. MR2311860 (2008b:42047)

2. C. K. Chui and J. A. Lian, Construction of compactly supported symmetric and antisymmetric orthonormal wavelets with scale =3, Appl. Comput. Harmon. Anal., 2 (1995), 21-51. MR:1313097(95m:42042)

3. I. Daubechies, Ten Lectures on Wavelets. CBMS-NSF Regional Conference Series in Applied Mathematics, 61, SIAM, Philadelphia, PA, 1992. MR1162107 (93e:42045)

4. J. Geronimo, D. P. Hardin, and P. Massopust, Fractal functions and wavelet expansions based on several scaling functions, J. Approx. Theory, 78 (1994), 373-401. MR.1292968(95h:42033)

5. B. Han, Symmetric orthonormal scaling functions and wavelets with dilation factor $4, A d v$. Comput. Math., 8 (1998), 221-247. MR1628249 (99d:42060)

6. B. Han, Matrix extension with symmetry and applications to symmetric orthonormal complex M-wavelets, J. Fourier Anal. Appl., 15 (2009), 684-705. MR2563779 (2010j:42076)

7. B. Han, Pairs of frequency-based nonhomogeneous dual wavelet frames in the distribution space, Appl. Comput. Harmon. Anal., 29 (2010), 330-353. MR2672230 (2011e:42071)

8. B. Han, Symmetric orthogonal filters and wavelets with linear-phase moments, J. Comput. Appl. Math., 236 (2011), no. 4, 482-503, doi:10.1016/j.cam.2011.06.008. MR2843033

9. B. Han and H. Ji, Compactly supported orthonormal complex wavelets with dilation 4 and symmetry, Appl. Comput. Harmon. Anal., 26 (2009), 422-431. MR2503314(2010c:42076)

10. B. Han and X. Zhuang, Matrix extension with symmetry and its application to symmetric orthonormal multiwavelets, SIAM J.Math. Anal., 42(5) (2010), 2297-2317. MR.2729441 (2011k:42074)

11. B. Han, S.-G. Kwon and X. Zhuang, Generalized interpolating refinable function vectors, $J$. Comput. Appl. Math., 227 (2009), 254-270. MR2510886 (2010c:42077)

12. B. Han and X. Zhuang, Analysis and construction of multivariate interpolating refinable function vectors, Acta Appl. Math., 107 (2009), 143-171. MR2520014(2010d:42064)

13. H. Ji and Z. Shen, Compactly supported (bi)orthogonal wavelets generated by interpolatory refinable functions, Adv. Comput. Math., 11 (1999), 81-104. MR1706397 (2000g:42052)

14. A. Klappenecker, Algebraic wavelet filters, Int. J. Imag. Syst. Tech., 7(3) (1996), 166-169.

15. W. Lawton, S. L. Lee, and Z. Shen, An algorithm for matrix extension and wavelet construction, Math. Comp., 65 (1996), 723-737. MR1333319 (96g:65018)

16. Q. Mo and S. Li, Symmetric tight wavelet frames with rational coefficients, Appl. Comput. Harmon. Anal., 31 (2011), 249-263.

17. A. Petukhov, Construction of symmetric orthogonal bases of wavelets and tight wavelet frames with integer dilation factor, Appl. Comput. Harmon. Anal., 17 (2004), 198-210. MR 2082158 (2005h:42076)

18. Q. Y. Sun, N. Bi, and D. R. Huang, An Introduction to Multiband Wavelets, Zhejiang Univ. Press, 2001.

19. P. P. Vaidyanathan, Multirate Systems and Filter Banks, Prentice Hall, Englewood Cliffs, New Jersey, 1993.

20. D. C. Youla and G. Gnavi, Notes on $n$-dimensional system theory, IEEE Trans. Circuits and Systems, 26 (1979), 105-111. MR 521657 (80b:93038) 
Department of Mathematical and Statistical Sciences, University of Alberta, Edmonton, Alberta, Canada T6G 2G1

URL: http://www .ualberta.ca/ bhan

E-mail address: bhan@math.ualberta.ca

Institut für Mathematik, Technische Universität, 10623 Berlin, Germany

URL: http://www.ualberta.ca/ ${ }^{\sim}$ xzhuang

E-mail address: xzhuang@math.tu-berlin.de 